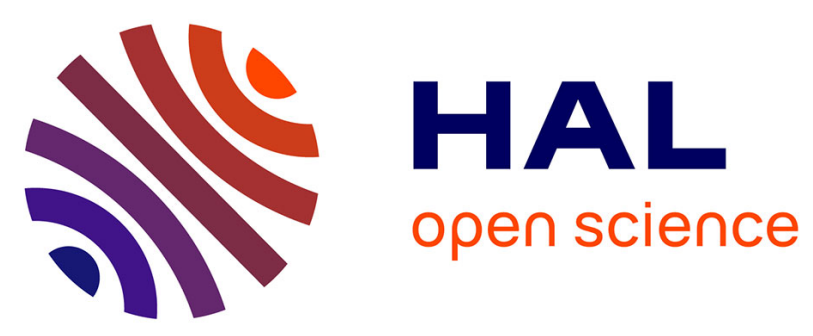

\title{
Distribution area of the two floral morphs of Nigella damascena L. (Ranunculaceae): A diachronic study using herbarium specimens collected in France
}

Catherine Damerval, Wafa Ben Othman, Domenica Manicacci, Florian Jabbour

\section{To cite this version:}

Catherine Damerval, Wafa Ben Othman, Domenica Manicacci, Florian Jabbour. Distribution area of the two floral morphs of Nigella damascena L. (Ranunculaceae): A diachronic study using herbarium specimens collected in France. Botany Letters, 2018, 165 (3-4), pp.396-403. 10.1080/23818107.2017.1422437 . hal-02187136

\section{HAL Id: hal-02187136 \\ https://hal.sorbonne-universite.fr/hal-02187136}

Submitted on 17 Jul 2019

HAL is a multi-disciplinary open access archive for the deposit and dissemination of scientific research documents, whether they are published or not. The documents may come from teaching and research institutions in France or abroad, or from public or private research centers.
L'archive ouverte pluridisciplinaire HAL, est destinée au dépôt et à la diffusion de documents scientifiques de niveau recherche, publiés ou non, émanant des établissements d'enseignement et de recherche français ou étrangers, des laboratoires publics ou privés. 
Distribution area of the two floral morphs of Nigella damascena L. (Ranunculaceae): A diachronic study using herbarium specimens collected in France

Catherine Damerval $^{\mathrm{a}}$, Wafa Ben Othman ${ }^{\mathrm{b}}$, Domenica Manicaccic ${ }^{\mathrm{c}}$, Florian Jabbour ${ }^{\mathrm{b}}$

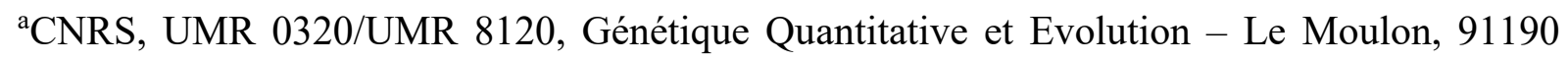

Gif-sur-Yvette, France; 'buséum national d'Histoire naturelle, Institut de Systématique, Évolution, Biodiversité, UMR 7205 ISYEB MNHN/CNRS/UPMC/ EPHE, Sorbonne Universités, 57 rue Cuvier, CP 39, 75005 Paris, France; 'Univ. Paris Sud, UMR 0320/UMR 8120, Génétique Quantitative et Evolution - Le Moulon, 91190 Gif-sur-Yvette, France

Word count: 4203

\begin{abstract}
Perianth dimorphism in Nigella damascena (Ranunculaceae) has been documented since four centuries, and its genetic basis has started to be studied almost one century ago. Based on herbarium collections, we reconstructed the evolution of the distribution area of the wild-type (perianth composed of a calyx and nectariferous petals) and mutant (apetalous) floral morphs in mainland France during the last two centuries. We showed that the collection localities of the wild-type morph are mostly concentrated in the southern coastal areas of France, whereas the collection localities of the mutant morph were less numerous but more scattered in France. The collection effort of both morphs peaked in the second half of the $19^{\text {th }}$ century and continuously decreased afterwards. Results are interpreted taken into account the biases in the constitution of a herbarium collection. Knowledge about the spatial distribution and the frequency of occurrence of wild-type and mutant populations through time will help botanists
\end{abstract}


and evolutionary biologists investigate the origin and ecological grounds for the maintenance and propagation of the apetalous morph in the wild.

\section{Keywords}

Collection bias; collecting effort; floral dimorphism; love-in-a-mist; museum time-series; natural history collection

\section{Introduction}

Floral dimorphism has intrigued botanists and evolutionary biologists since centuries. Darwin devoted a book to this topic, focusing on heterostylous Primula flowers (Darwin 1897). Nigella damascena L. (Ranunculaceae), or love-in-a-mist, is a herbaceous species mainly distributed in the Mediterranean Basin, whose ancient use by human populations is well documented (Heiss and Oeggl 2005). In this species, two types of flowers are known at least since the end of the $16^{\text {th }}$ century (Clusius 1601). The wild-type flower has a perianth composed of a pentamerous petaloid calyx and a series of 5-10 pouch-shaped nectariferous petals. By contrast, the unipartite perianth of the mutant type consists of numerous petaloid tepals (Deroin et al. 2015; Jabbour et al. 2015). The monogenic control of the dimorphism was first established by Toxopeus (1927) based on segregation analyses, and its molecular basis was unraveled almost one hundred year later (Gonçalves et al. 2013; Zhang et al. 2013). For simplicity, we will adopt the terminology of Gonçalves et al. (2013) to designate the wildtype (homozygous dominant or heterozygous for the combination of alleles controlling the dimorphism) and mutant (homozygous recessive) floral morphs, called [P] and [T], respectively. Human selection for plants with conspicuous flowers harbouring supernumerary petaloid organs likely played an important role in the long-term maintenance of the [T] phenotype, but $[\mathrm{T}]$ populations are also expected to occur in the wild because individuals are 
self-fertile and plants can easily escape from gardens and become naturalized in favorable environments.

Herbarium collections are repositories of past and current plant biodiversity. Compiling the data held by all the herbarium specimens of a species allows reconstructing the evolution of its distribution area through time. In the case of $N$. damascena, knowledge about the spatial distribution and the frequency of occurrence of $[\mathrm{P}]$ and $[\mathrm{T}]$ populations through time would help botanists and evolutionary biologists investigate the origin and ecological grounds for the maintenance and propagation of the $[\mathrm{T}]$ morph in the wild. It should be possible to examine whether [T] populations can persist per se in the wild or if they are consistently found in the neighborhood of towns and villages, suggesting constant gene flow from gardens; alternatively, $[\mathrm{T}]$ individuals could consistently be found in the vicinity of $[\mathrm{P}]$ individuals, suggesting that the presence of nectariferous flowers in $[\mathrm{P}]$ plants may favor pollination and reproductive success of $[\mathrm{T}]$ plants with flowers lacking pollinator reward, and/or that the mutation is slightly deleterious and preferentially maintained in the heterozygous state. A last use of distribution maps would be to identify localities where $N$. damascena individuals from each morph could be currently collected.

To get insight into these topics, we built a quasi-exhaustive database of the world collection of $N$. damascena herbarium specimens. For this special issue of Botany Letters, we present the results concerning mainland France, explaining the database structure and the protocol to select the herbarium specimens for georeferencing and building the temporal sequence of distribution maps. We observe different distributions of the two morphs in mainland France, and discuss possible causes.

\section{Materials and Methods}

\section{Data collection and database construction}


We built a database gathering the information found on herbarium specimens of $N$.

damascena (Fig. 1) from 39 herbaria, from the Mediterranean Basin or from other regions but housing large amounts of Mediterranean species or species growing in areas with a Mediterranean climate. The herbaria sent specimens on loan to P (Paris Herbarium), where they were studied. They were photographed for the record. Alternatively, the herbaria sent images of their collection of $N$. damascena.

The data gathered from the biological specimen (Fig. 1A-B and 1D) and from the label (Fig. 1C) are organized into 16 fields: (A) herbarium code; (B) barcode or reference in the herbarium; (C) collector's name; (D) collection number; $(E-G)$ day, month, and year of collection; $(\mathrm{H})$ country of origin, transcribed from the label or interpreted; current country names are provided; (I) collection locality; (J) other notes on the label (in the original language); (K) label mentioning that the specimen was in cultivation at the time of collection (yes/no); (L) assumption that the specimen was in cultivation at the time of collection (yes/no); (M) presence of flower(s) (yes/no); (N) flower morph (P/T, Fig. 1B and 1D); (O) presence of capsule(s) (yes/no); (P) GPS coordinates in decimal degrees, in the WGS 84 system. In some cases, GPS coordinates were available directly from the label. In most cases, localities were georeferenced by us using Google Maps (Google Inc., Mountain View, CA, USA). The collection locality could be precisely specified on the label (e.g. "Gorges d'Estrabols, commune de Salvagnac, canton de Villeneuve"), allowing precise GPS coordinates to be determined (in red in Supplementary Table 2). Less precisely described collection sites, for example just mentioning the closest city, were also attributed GPS coordinates (in blue in Supplementary Table 2). In other cases, e.g. when only the country of origin is specified or when the plant was in cultivation when collected with no mention of the seed origin, localization data was considered unavailable and $(\mathrm{P})$ cells were marked with a double-slash (//). Except for (H) and (L), all data are transcribed from the label without 
interpretation or come from the observation of the specimen. Label data was provided as a csv file by the following herbaria: BC, BEI, BM, E, FR, HUJ, and MA; it was double-checked before being included in our database.

\section{Diachronic study of the distribution of Nigella damascena in France}

For the present study, we focused on data relative to all specimens collected in France (654 specimens; Supplementary Table 1, Fig. 1), recorded from 20 herbaria (B (Berlin), BC (Barcelona), BEI (Beirut), BM (London), CAI (Cairo), E (Edinburgh), FI (Florence), FR (Frankfurt), G (Geneva), HUJ (Jerusalem), K (Kew), MA (Madrid), MEL (Melbourne), MPU (Montpellier), P (Paris), PAL (Palermo), S (Stockholm), TO (Torino), UPS (Uppsala), W (Vienna)); herbarium codes follow Thiers (2017). For the analysis of the distribution of the two morphs over time, we did not consider specimens without flowers (or with flowers compressed in such a way that the floral morph was not distinguishable), duplicate specimens (belonging to the same collection, i.e. same collector and collection number) and specimens with no collection year. We considered two datasets extracted from the database:

(i) the georeferenced dataset excludes specimens with a double slash in (P), and gathered 409 specimens from 18 herbaria (all specimens from BEI and CAI herbaria were discarded). Among these specimens, 12 were composed of two different collections (different combination [collector + collection number]). After splitting these accessions into two, the final dataset consisted of 421 entries. Supplementary Table 2 shows the collections that were used to build the distribution maps. We classified the entries into four time intervals ([18001849], [1850-1899], [1900-1949], [1950-1999]), and into two categories ([P] and [T] morph) within each interval. When the collection date is an interval, the latest year was considered. After the sorting process described above, no specimen collected in France after 2000 was included in the dataset. Maps were built using SimpleMappr (Shorthouse 2010). 
(ii) the $[\mathrm{P}] /[\mathrm{T}]$ dataset was built to compare the number of collected specimens from each morph among each 50-year period. It includes the cultivated specimens and the specimens that could not be georeferenced, and comprises 469 entries.

\section{Results}

\section{More than two centuries of biogeographical data captured in herbarium specimens}

The oldest and most recent French specimens of $N$. damascena included in our database were collected in 1784 by an unknown collector and in 2015 by F. Jabbour, respectively. These specimens were both cultivated (in "Jardin de Sally" and in Gif-sur-Yvette (see Jabbour et al. 2015), respectively), and hence not georeferenced. The whole dataset consists of 654 collections. The three herbaria that contributed most in terms of number of collections were $\mathrm{P}$ (Paris, 313 collections), MPU (Montpellier, 105) and G (Geneva, 43). The floral morph could be identified on 469 specimens $(371[\mathrm{P}]$ and $98[\mathrm{~T}])$ and 421 specimens $(352[\mathrm{P}]$ and $69[\mathrm{~T}])$ could be georeferenced. The three most active collectors of $N$. damascena specimens in France were Elisée Reverchon (1835-1914; 11 specimens), Florian Jabbour (1983-; 9 specimens), and Brother Sennen (Etienne Marcellin Granié-Blanc, 1861-1937; 8 specimens).

The collection localities of the [P] morph are mostly concentrated in the southern coastal areas of France

Figure 2 shows the evolution of the distribution area of both floral morphs of $N$. damascena since 1800. Individuals from the $[\mathrm{P}]$ morph have mostly been collected from the southern half of France. Collection localities for this morph are particularly concentrated in the coastal areas of SE France (Provence-Alpes-Côte d'Azur, Languedoc-Roussillon and Corsica regions) and SW (Poitou-Charentes region). In the second half of the $19^{\text {th }}$ century, four areas from which $[\mathrm{P}]$ specimens are mostly collected could be identified, namely, from West to 
East, the Charente-Maritime department, the Hérault department, the Rhône Valley and the Alpes-Maritimes department. For the $[\mathrm{T}]$ morph, the collection localities are less numerous but more scattered than for the $[\mathrm{P}]$ morph. Additionally, they tend to be more northerly distributed in France.

\section{A peak of collection in the second half of the $19^{\text {th }}$ century}

The collection of both morphs was the highest between 1850 and 1899 (233, about half of the specimens of the dataset), before decreasing continuously afterwards (Fig. 3A). The peak also coincides with more individuals from both morphs collected from the northern half of France. The collection of cultivated specimens, even though less numerous, follows the same temporal trend as the wild ones (Fig. 3B). In the wild collections, [P] morph individuals are more frequent than $[\mathrm{T}]$ morph ones in all time intervals (2.6 to 10 times more). The proportion of [T] morph individuals was the highest in the first half of the $20^{\text {th }}$ century, reaching $28 \%$. In contrast to the wild collection, more $[\mathrm{T}]$ than $[\mathrm{P}]$ individuals were found in the cultivated collection at each time period (Fig. 3B). During the last two centuries, 28 of the c. 300 collectors of $N$. damascena collected both floral morphs, but only 15 of them collected both morphs in the same locality.

\section{Discussion}

\section{Caution required when analyzing herbarium collection records}

A collection of natural history is by nature biased. Not all the biodiversity can be captured in time and space, and the representativeness of a collection depends on the spatio-temporal uniformity of the collection effort (Auderset Joye et al. 2002). Many collectors collect objects of natural history in the places where they live or travel, and they can be biased towards or against unusual objects (e.g., with an unusual morphology or spatial occurrence). They can 
collect in priority individuals with unusual phenotypes in order to have a record in their own collection, or they can ignore them because they deviate from the standard morphology. Indeed, botanists can purposely ignore plants considered as weeds and plants that are locally common. Hence a herbarium collection can paradoxically lack plants that are common or present at a given locality for long. Knowing these limitations, herbarium collection data should not be over-interpreted. They give a picture of biodiversity at certain times and places, filtered by collectors' interest that may vary according education and societal context. For a given species, a way to partly circumvent bias is to consider the most exhaustive set of herbarium collections from the highest number of herbaria. This is what we intended to do for N. damascena.

\section{The evolution of Nigella damascena collection effort in France}

In France, the collection effort concerning $N$. damascena has not been uniform during the last two centuries. After a peak in the second half of the $19^{\text {th }}$ century, the number of specimens added to collections of natural history decreased continuously. Does it reveal that $N$. damascena distribution area has expanded and then shrunk down, or that the collection effort has been lower during the last decades? We tend to favor the second hypothesis. Indeed, we cannot figure out any natural cause that could explain a decrease in abundance in the wild, since this species has been common in France (see SIFlore and INPN databases; Just et al. 2015; Muséum national d'Histoire Naturelle 2003-2017; Fig. 4). However, the decrease in specimen number collected in the first half of the $20^{\text {th }}$ century could be due to the impact of the two world wars on the activity of the scientists (Le Bras et al. 2015). Furthermore, we witness nowadays a general decline in collecting due to less funding for research in the field of natural history and the societal perception that collecting local flora is unnecessary (Renner and Rockinger 2016). 
The fact that $N$. damascena has often been collected next to areas with high human population density could be due to the local presence of many collectors and to the naturalization of populations after escaping from gardens. Whilst this explanation could stand for the Parisian region, we do not think it is valid for the whole French territory, as N. damascena has not been heavily collected from many densely inhabited areas in northern France. Thus, we believe that the distribution of the species at the Atlantic coast, at the Rhône Valley and at the Mediterranean coast represent its true distribution area. The data made available online by SIFlore (Just et al. 2015), gathering distributional data for vascular plants in France from herbarium records but also from the literature and data from botanical conservatories and covering the last five centuries (from 1545 to 2014) and by the INPN (Muséum national d'Histoire Naturelle 2003-2017) are in accordance with our results, considering that these datasets distinguishes neither the $[\mathrm{P}]$ from the $[\mathrm{T}]$ morph nor wild from cultivated specimens (Figure 4).

The morph $[\mathrm{T}]$ has been less collected than the $[\mathrm{P}]$ morph over the last couple of centuries suggesting that such populations are less common in the wild than the $[\mathrm{P}]$-morph populations $(72 / 430=17 \%)$. One-tenth of the collectors of $N$. damascena collected both floral morphs, and more than half of them found both morphs in the same locality. This suggests that at least $21 \%(15 / 72)$ of the $[\mathrm{T}]$ phenotype appears in mixed $[\mathrm{P}]-[\mathrm{T}]$ populations.

Additionally, the two morphs are not equally distributed in France. Relatively more [T] specimens have been collected in the northern part of France and there is no reason to think that the collectors mostly living or botanizing in the northern half of France were more sensitive to unusual phenotypes. The presence of [T] populations further away from the Mediterranean and the Atlantic coasts could be due to the naturalization of populations in the vicinity of towns and villages after escaping from the gardens. The fact that most cultivated 
specimens, which are enriched in [T] morphs, were also collected from the northern half of France is consistent with this hypothesis.

\section{A dataset valuable for diachronic evo-devo studies}

Museum time-series (set of specimens from the same species collected at different times) are the basic material for floras and conservation studies. Thanks to the information gathered on the collection label, the distribution area of a single species can be traced over time (e.g., Auderset Joye, Castella, and Lachavanne 2002). Such diachronic studies can be conducted at the scale of some centuries and do not result from any inference-based analysis. They differ from studies of historical biogeography in which older (thousand- to million-year old) dispersal events are inferred from the distribution areas of the extant taxa and the topology of a phylogenetic tree.

The distribution maps we built are based on the georeferencing of the collection localities of 421 individuals across two hundred years. Among this set of individuals, only three could be georeferenced with high precision, meaning the maps presented in Fig. 2 mostly show broad localities. However, comparing maps obtained from $[\mathrm{P}]$ and $[\mathrm{T}]$ specimens can allow us targeting localities where populations of both morphs could currently coexist. Such natural populations would be required for in situ long-term ecological studies.

Ancient DNA (i.e. extracted from relatively old herbarium specimens) has been sequenced to track invasion routes (Matsuhashi et al. 2016) or analyse the evolution of the genetic diversity of a plant pathogen (Li et al. 2007) through time. In the case of $N$. damascena, mining our database for old and modern specimens from different origins will make it possible to test whether the molecular basis for the [T] phenotype is identical at the scale of the Mediterranean Basin as a whole, during the last two centuries. This research is currently under way in our team. 


\section{Acknowledgments}

We thank the curators and staff members of B (Robert Vogt and Ulrike Starck), BC (Neus Nualart Dexeus), BEI (Nada Sinno Saoud), BM (Mark Carine and Jacek Wajer), CAI (Hasnaa Hosni), E (Lesley Scott), FI (Lorenzo Cecchi), FR (Georg Zizka and Rainer Doering), G (Cyrille Chatelain), HUJ (Jotham Ziffer-Berger), K (Marie-Hélène Weech), MA (Charo Noya Santos), MEL (Helen Barnes), MPU (Caroline Loup), PAL (Gianniantonio Domina), S (Arne Anderberg), TO (Laura Guglielmone), UPS (Mats Hjertson), W (Ernst Witek), for sending herbarium specimens on loan and/or sending digital images. We thank the staff members of $\mathrm{P}$ Herbarium for their technical help and the Institute for Systematics, Evolution and Biodiversity for funding the internship of $\mathrm{W}$. Ben Othman. We also thank two anonymous reviewers for their critical and helpful comments and suggestions.

\section{Disclosure statement}

No potential conflict of interest was reported by the authors.

\section{Notes on contributors}

Catherine Damerval is senior researcher at Centre national de la Recherche Scientifique. Her research deals with evo-devo of the flower in Ranunculales.

Wafa Ben Othman is an agronomist, recently graduated from AgroParisTech.

Domenica Manicacci is an assistant professor at Université Paris-Sud. She is a population geneticist working on different angiosperm models such as maize and Nigella damascena. 
Florian Jabbour is an associate professor at the Muséum national d'Histoire Naturelle and a curator at Paris Herbarium. His research focuses on the evolution of plant taxa and plant shapes. He is editor for Botany Letters.

For this publication, $\mathrm{CD}, \mathrm{WBO}, \mathrm{DM}$ and FJ gathered data, $\mathrm{CD}$ and $\mathrm{FJ}$ analyzed the results and wrote a first version of the manuscript, and all coauthors contributed to the last version of the manuscript.

\section{References}

Auderset Joye, D., E. Castella, and J. B. Lachavanne. 2002. “Occurrence of Characeae in Switzerland over the last two centuries (1800-2000)." Aquatic Botany 72 (3): 369-385. doi:10.1016/S0304-3770(01)00211-X.

Clusius, C. 1601. "Melanthium pleno flore.” In Rariorum Plantarum Historia, 207-208. Antwerp: Ex officina Plantiniana Apud Ioannem Moretum.

Darwin, C. 1897. The different forms of flowers on plants of the same species. New York: D. Appleton.

Deroin, T., C. Damerval, M. Le Guilloux, and F. Jabbour. 2015. "Floral vascular patterns of the double-flowered and wild-type morphs of Nigella damascena L. (Ranunculaceae)." Modern Phytomorphology 7: 13-20.

Gonçalves, B., O. Nougué, F. Jabbour, C. Ridel, H. Morin, P. Laufs, D. Manicacci, and C. Damerval. 2013. An APETALA3 homolog controls both petal identity and floral meristem patterning in Nigella damascena L. (Ranunculaceae). The Plant Journal 76 (2): 223-235. doi:10.1111/tpj.12284. 
Heiss, A. G., and K. Oeggl. 2005. The oldest evidence of Nigella damascena L.

(Ranunculaceae) and its possible introduction to central Europe. Vegetation History and Archaeobotany 14 (4): 562-570. doi:10.1007/s00334-005-0060-4.

Jabbour, F., M. Udron, M. Le Guilloux, B. Gonçalves, D. Manicacci, S. Nadot, and C. Damerval. 2015. "Flower development schedule and AGAMOUS-like gene expression patterns in two morphs of Nigella damascena (Ranunculaceae) differing in floral architecture.” Botanical Journal of the Linnean Society 178 (4): 608-619. doi:10.1111/boj.12297.

Just, A., J. Gourvil, J. Millet, V. Boullet, T. Milon, I. Mandon, and B. Dutrève. 2015. "SIFlore, a dataset of geographical distribution of vascular plants covering five centuries of knowledge in France: Results of a collaborative project coordinated by the Federation of the National Botanical Conservatories." PhytoKeys 56: 47-60. doi: 10.3897/phytokeys.56.5723.

Le Bras, G., M. Pignal, M. L. Jeanson, S. Muller, C. Aupic, B. Carré, G. Flament, M. Gaudeul, C. Gonçalves, VR. Invernón, F. Jabbour, E. Lerat, P. P. Lowry, B. Offroy, E. P. Pimparé, O. Poncy, G. Rouhan, and T. Haevermans. 2017. “The French Muséum national d'histoire naturelle vascular plant herbarium collection dataset." Scientific Data 4: 170016. doi:10.1038/sdata.2017.16.

Li, W., Q. Song, R. H. Brlansky, and J. S. Hartung. 2007. “Genetic diversity of citrus bacterial canker pathogens preserved in herbarium specimens." Proceedings of the National Academy of Sciences 104 (47): 18427-18432. doi:10.1073/pnas.0705590104.

Matsuhashi, S., H. Kudoh, M. Maki, M. Cartolano, M. Tsiantis, T. Itagaki, and S. Sakai. 2016. "Invasion history of Cardamine hirsuta in Japan inferred from genetic analyses of herbarium specimens and current populations.” Biological Invasions 18 (7): 1939-1951. doi:10.1007/s10530-016-1139-9. 
Muséum national d'Histoire naturelle. 2003-2017. “Inventaire National du Patrimoine Naturel." https://inpn.mnhn.fr

Renner, S. S., and A. Rockinger. 2016. "Is plant collecting in Germany coming to an end?" Willdenowia 46 (1): 93-97. doi:10.3372/wi.46.46106.

Shorthouse, D.P. 2010. "SimpleMappr, an online tool to produce publication-quality point maps." http://www.simplemappr.net

Thiers, B. 2017. "Index Herbariorum: A global directory of public herbaria and associated staff, New York Botanical Garden's Virtual Herbarium.” http://sweetgum.nybg.org/science/ih/

Toxopeus, H. J. 1927. “Erblichkeitsuntersuchungen an Nigella damascena L.” Genetica 9: $341-440$.

Zhang, R., C. Guo, W. Zhang, P. Wang, L. Li, X. Duan, Q. Du, L. Zhao, H. Shan, S. A. Hodges, E. M. Kramer, Y. Ren, and H. Kong. 2013. "Disruption of the petal identity gene APETALA3-3 is highly correlated with loss of petals within the buttercup family (Ranunculaceae)." Proceedings of the National Academy of Sciences 110 (13): 50745079. doi:10.1073/pnas.1219690110.

\section{Figure legends}

Figure 1. Herbarium material providing data about the floral morph and the collection date and locality. (A) Herbarium specimen of Nigella damascena (barcode P02575070) presenting individuals from the $[\mathrm{P}]$ floral morph. (B) A focus on the most mature flower. The white arrows point at two of the petals. (C) A focus on the label showing the collection number, the determination provided by the collector, the collection locality and date, and the name of the 
collector. (D) A flower from the [T] morph, from the specimen P00195185. Digital images of both specimens can be downloaded using the following links: http://coldb.mnhn.fr/catalognumber/mnhn/p/p02575070 and http://coldb.mnhn.fr/catalognumber/mnhn/p/p00195185.

Figure 2. Distribution area of Nigella damascena in France during the last two centuries, broken up in 50-year periods. (A-D) Distribution area of the $[\mathrm{P}]$ morph. $(\mathrm{E}-\mathrm{H})$ Distribution area of the $[\mathrm{T}]$ morph.

Figure 3. Frequency of $[\mathrm{P}]$ and $[\mathrm{T}]$ morphs of Nigella damascena in a Museum time-series spanning two centuries. (A) Wild specimens. (B) Cultivated specimens.

Figure 4. Distribution maps of Nigella damascena (both morphs, wild and cultivated) based on national and regional databases. (A) Number of observations adapted from the SIFlore dataset. (B) Collection dates adapted from the SIFlore dataset. (C) Occurrence data adapted from the INPN dataset.

Supplementary Table 1. Herbarium specimens of Nigella damascena collected in France, label data transcribed and interpreted. For each specimen, the data is organized into 16 fields: (A) Herbarium code. (B) Barcode or reference in the herbarium. (C) Collector's name. (D) Collection number. (E-G) Day, month and year of collection. $(\mathrm{H})$ Country of origin, transcribed from the label or interpreted. Current country names are provided. (I) Collection locality. (J) Other notes on the label. (K) Is there anything on the label specifying that the specimen was in cultivation at the time of collection? (L) Assumption that the specimen was in cultivation at the time of collection. (M) Presence of flower(s). (N) Flower morph. (O) 
Presence of capsule(s). (P) GPS coordinates, transcribed from the label or inferred. Except for $(\mathrm{H})$ and $(\mathrm{L})$, all data are transcribed from the label without interpretation or come from the observation of the specimen.

Supplementary Table 2. This table corresponds to Supp. Table 1 from which all the entries not taken into consideration (see our sorting procedure in the Materials and Methods section) for building the distribution maps were excluded. 


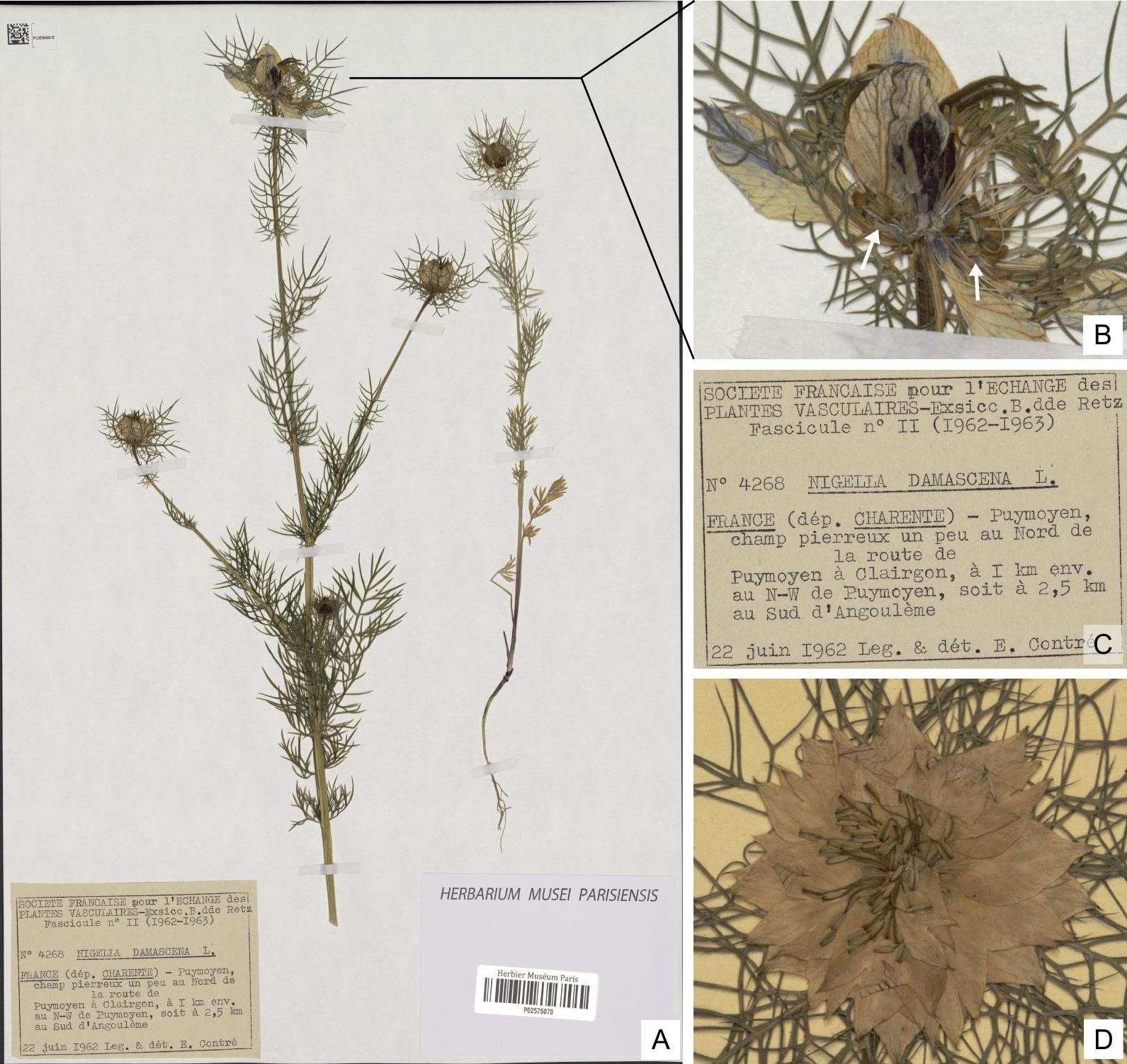




\begin{tabular}{|c|c|c|c|c|c|}
\hline \multirow[t]{2}{*}{ Herbarium } & \multicolumn{2}{|c|}{ Barcode or referenc $€$ Collector } & \multicolumn{2}{|l|}{ Collection number } & \multirow{2}{*}{$\begin{array}{l}\text { Collectior } \\
\text { Month }\end{array}$} \\
\hline & & & & Day & \\
\hline B & В 100525356 & F. Schultz & 408 & 26 & 5 \\
\hline B & В 100525364 & F. Schultz & 408 & 26 & 5 \\
\hline B & В 100525361 & Canut & 391 & 29 & 5 \\
\hline B & В 100525362 & Canut & 391 & 29 & 5 \\
\hline B & В 100525394 & Héry & s.n. & n.a. & n.a. \\
\hline B & В 100525433 & Dr. Wirtgen fil & s.n. & 23 & 7 \\
\hline B & В 100525360 & leg. ign. & 391 & n.a. & 6 \\
\hline B & B 100525432 & leg. ign. & 26240 & 10 & 8 \\
\hline B & В 100525359 & H.L. Krause & 30549 & 9 & 7 \\
\hline B & В 100525357 & H. Beger & s.n. & 11 & 6 \\
\hline B & В 100525358 & H. Beger & s.n. & 12 & 6 \\
\hline B & В 100525355 & P. Bamps & 9002 & 12 & 6 \\
\hline $\mathrm{BC}$ & 135239 & leg. ign. & s.n. & 20 & 5 \\
\hline BC & 135240 & R. Linder & s.n. & 11 & 7 \\
\hline $\mathrm{BC}$ & 135238 & A. Frizac & s.n. & n.a. & n.a. \\
\hline BC & $833041-2$ & Sennen & s.n. & 27 & 4 \\
\hline $\mathrm{BC}$ & $833040-2$ & C.-A Gérard & s.n. & 12 & 6 \\
\hline BC & $833040-2$ & C.-A Gérard & s.n. & 12 & 6 \\
\hline $\mathrm{BC}$ & 1523 & Marnac & s.n. & 19 & 6 \\
\hline $\mathrm{BC}$ & $833042-2$ & Sennen & s.n. & n.a. & 6 \\
\hline $\mathrm{BC}$ & 1543 & L. Samat & 6059 & 25 & 5 \\
\hline$B C$ & $373730-3$ & G. Gavelli & s.n. & 5 & 6 \\
\hline $\mathrm{BC}$ & 135237 & Gerclard & s.n. & 4 & 9 \\
\hline BC & 834564 & P. Bamps & 15013 & 12 & 6 \\
\hline BEI & n.a. & J.J.M. & s.n. & 12 & 5 \\
\hline BM & BM001209513 & E. Bourgeau & 2 & 25 & 5 \\
\hline BM & BM001209454 & J.M. Delalande & s.n. & n.a. & 9 \\
\hline BM & BM001209450 & M. Prost & s.n. & n.a. & 6 \\
\hline BM & BM000984476 & J. Revellière & 8 & 14 & 6 \\
\hline BM & BM001209512 & J.C. Ducommun & s.n. & 26 & 5 \\
\hline BM & BM001209455 & F.S.A. Augé de Lassus & s.n. & 10 & 6 \\
\hline BM & BM001209511 & H. Hanry & 3003 & $6--3$ & $5--6$ \\
\hline BM & BM001209509 & E. Miciol & S.n. & 4 & 6 \\
\hline BM & BM000649099 & [illegible] & s.n. & 30 & 6 \\
\hline BM & BM000984501 & P.A. Kesselmeyer & s.n. & n.a. & 6 \\
\hline BM & BM000984475 & J-O. Debeaux & s.n. & 30 & 5 \\
\hline BM & BM001209453 & E.J. de Puyfol & s.n. & 13 & 5 \\
\hline BM & BM001209451 & L. Anthouard & s.n. & 9 & 6 \\
\hline BM & BM001209452 & A. Cartier & 1487 & 20 & 5 \\
\hline BM & BM001209505 & E. Marçais & s.n. & n.a. & $6--7$ \\
\hline BM & BM001209412 & E. Reverchon & s.n. & 14 & 6 \\
\hline BM & BM001209507 & N.D. Simpson & 36543 & n.a. & 5 \\
\hline
\end{tabular}




\begin{tabular}{|c|c|c|c|c|}
\hline BM & BM000984490 & R.P. Murray & s.n. & 10 \\
\hline BM & BM000984489 & R.P. Murray & s.n. & 15 \\
\hline BM & BM000984488 & J. Bruneau & s.n. & 19 \\
\hline BM & BM001209508 & J. Lloyd & s.n. & 20 \\
\hline BM & BM001209510 & E.J.A. Gadeceau & s.n. & 2 \\
\hline BM & BM001209514 & V. Bonafons & s.n. & n.a. \\
\hline BM & BM001209506 & Septimin-Donat & 111 & 8 \\
\hline BM & BM000649100 & P.R. Lowe & s.n. & 22 \\
\hline BM & BM000649110 & J. Roffey & s.n. & 15 \\
\hline BM & BM001209515 & L. Samat & 6059 & 25 \\
\hline BM & BM001209447 & L. Samat & 6059 & 25 \\
\hline BM & BM000649076 & P.J.R. Bamps & 9002 & 12 \\
\hline BM & BM000984492 & A.A. du Petit-Thouars & s.n. & n.a. \\
\hline BM & BM000984491 & F.I.X. Rugel & s.n. & n.a. \\
\hline CAI & n.a. & P.F.A. Irat & 408 & 26 \\
\hline $\mathrm{E}$ & E00743401 & Seringe & s.n. & n.a. \\
\hline E & E00743450 & G.W. Arnott & s.n. & 18 \\
\hline$E$ & E00743402 & Seringe & 7 & 18 \\
\hline$E$ & E00743403 & Seringe & 127 & 22 \\
\hline E & E00743439 & F. Kuyes (?) & s.n. & n.a. \\
\hline E & E00743413 & F. Rugel & s.n. & n.a. \\
\hline$E$ & E00743413 & F. Rugel & s.n. & n.a. \\
\hline$E$ & E00445049 & leg. ign. & s.n. & n.a. \\
\hline$E$ & E00743421 & J. Ball & s.n. & 22 \\
\hline E & E00743410 & O. Debeaux & s.n. & 24 \\
\hline$E$ & E00743409 & H. Hanry & s.n. & n.a. \\
\hline E & E00743408 & E. Reverchon & 340 & 10 \\
\hline E & E00743407 & Elisée Reverchon & 340 & 14 \\
\hline$E$ & E00743451 & E. Reverchon & s.n. & 17 \\
\hline E & E00743432 & E. Reverchon & s.n. & 19 \\
\hline E & E00743396 & Elisée Reverchon & s.n. & 19 \\
\hline$E$ & E00743419 & G. Bentham & s.n. & n.a. \\
\hline$E$ & E00743418 & W. Wilson Saunders & s.n. & n.a. \\
\hline$E$ & E00743419 & leg. ign. & s.n. & n.a. \\
\hline $\mathrm{FI}$ & DSN112 & Durando (?) & s.n. & 14 \\
\hline $\mathrm{FI}$ & DSN107 & Reuter & s.n. & n.a. \\
\hline $\mathrm{FI}$ & DSN439 & P.F.A. Irat & s.n. & n.a. \\
\hline $\mathrm{FI}$ & DSN417 & C. Marchesetti & 408 & 26 \\
\hline $\mathrm{FI}$ & DSN384 & A. Chabers (?) & s.n. & n.a. \\
\hline $\mathrm{FI}$ & DSN444 & Ch. Pontarlier & s.n. & n.a. \\
\hline $\mathrm{FI}$ & DSN108 & Parlatere & s.n. & 22 \\
\hline $\mathrm{FI}$ & DSN427 & Masson (?) & s.n. & 27 \\
\hline $\mathrm{FI}$ & DSN431 & H. Hervier-Basson & s.n. & 27 \\
\hline $\mathrm{FI}$ & DSN426 & A. Cartier & 1487 & 20 \\
\hline
\end{tabular}




\begin{tabular}{|c|c|c|c|c|c|}
\hline $\mathrm{FI}$ & DSN385 & A. Chabers & s.n. & 17 & 6 \\
\hline $\mathrm{FI}$ & DSN443 & V. Bach & s.n. & n.a. & 7 \\
\hline $\mathrm{FI}$ & DSN425 & Daides (?) & s.n. & n.a. & n.a. \\
\hline $\mathrm{FI}$ & DSN104 & Etienne Bourgeau & s.n. & n.a. & 5 \\
\hline $\mathrm{FI}$ & DSN445 & Franqueville & s.n. & n.a. & 12 \\
\hline $\mathrm{FI}$ & DSN446 & C. Flahault & s.n. & 14 & 6 \\
\hline $\mathrm{FI}$ & DSN423 & G. Gavelle & s.n. & 5 & 6 \\
\hline $\mathrm{FI}$ & DSN453 & P. Bamps & 9002 & 12 & 6 \\
\hline $\mathrm{Fl}$ & DSN448 & E. Levier & s.n. & n.a. & 5 \\
\hline FR & FR-0121334 & J.-C. Ducommun & s.n. & 26 & 5 \\
\hline FR & FR-0121331 & \multicolumn{2}{|c|}{ P.A. Kesselmeyer \& E. Ra s.n. } & 20 & 6 \\
\hline FR & FR-0121332 & A. Cartier & 1487 & 20 & 5 \\
\hline FR & FR-0030911 & Elisée Reverchon & 340 & 14 & 6 \\
\hline FR & FR-0030912 & Elisée Reverchon & 340 & 14 & 6 \\
\hline FR & FR-0121333 & N.Ch. Pontarlier & s.n. & 1 & 7 \\
\hline FR & FR-0121325 & J. de Vichet & s.n. & n.a. & $6--7$ \\
\hline FR & FR-0121340 & C. de Stefani & s.n. & n.a. & n.a. \\
\hline FR & FR-0121345 & Stifani & s.n. & 4 & 7 \\
\hline FR & FR-0121347 & R. Weber & s.n. & n.a. & 5 \\
\hline FR & FR-0121376 & Diomède & s.n. & n.a. & n.a. \\
\hline G & P6094975 & Choisy & s.n. & n.a. & n.a. \\
\hline G & P6095020 & Rodolph Rapin & s.n. & 5 & 6 \\
\hline G & P6094885 & Requien & s.n. & n.a. & n.a. \\
\hline G & P6014889 & Fauché & s.n. & n.a. & n.a. \\
\hline G & P6094976 & A. de St Hilaire & s.n. & n.a. & 6 \\
\hline G & P6094908 & Huet du Pavillon & s.n. & 22 & 5 \\
\hline G & P6014884 & J. Lloyd & s.n. & 23 & 6 \\
\hline G & P6014882 & Reuter & s.n. & n.a. & n.a. \\
\hline G & P6095091 & \multicolumn{2}{|c|}{ Dr. Friedrich Sigmund Al s.n. } & n.a. & 7 \\
\hline G & P6094977 & \multicolumn{2}{|c|}{ Dr. Friedrich Sigmund Al s.n. } & 4 & 7 \\
\hline G & P6095063 & Dr. Dupin & s.n. & n.a. & 6 \\
\hline G & P6094904 & E. Ayasse & s.n. & 4 & 5 \\
\hline G & P6095008 & E. Ayasse & s.n. & n.a. & 5 \\
\hline G & P6094978 & \multicolumn{2}{|c|}{ Dr. Friedrich Sigmund Al s.n. } & 10 & 6 \\
\hline G & P6095061 & Bélanger & s.n. & n.a. & n.a. \\
\hline G & P6094982 & Bélanger & s.n. & n.a. & n.a. \\
\hline G & P6095076 & Fasson & s.n. & 26 & 4 \\
\hline G & P6094909 & Alph. Moritz & s.n. & 10 & 5 \\
\hline G & P6094905 & Ch. Verrier Livardière & s.n. & 1 & 6 \\
\hline G & P6094973 & J.L.A. Bonneau & s.n. & 2 & 7 \\
\hline G & P6094887 & Dr. A. Bétrix & s.n. & 10 & 6 \\
\hline G & P6094985 & Bélanger & s.n. & n.a. & 5 \\
\hline G & P6095059 & Louis Amblard & s.n. & 20 & 8 \\
\hline G & P6095070 & Baraize, A. Perrier & s.n. & n.a. & 6 \\
\hline
\end{tabular}




\begin{tabular}{|c|c|c|c|c|c|}
\hline G & P6095069 & Roux, A. Perrier & s.n. & n.a. & n.a. \\
\hline G & P6094888 & W. Gugelmann & $37 / 224$ & 18 & 6 \\
\hline G & P6094911 & Louis-André Girod & s.n. & n.a. & 6 \\
\hline G & P6094907 & M.K. Faust & s.n. & 26 & 5 \\
\hline G & P6014916 & D. Champagneux & s.n. & 26 & 5 \\
\hline G & P6094996 & Simone Vautier & s.n. & 13 & 6 \\
\hline G & P6094928 & Duperrex-Weber & s.n. & 14 & 6 \\
\hline G & P6094927 & Duperrex-Weber & s.n. & 8 & 6 \\
\hline G & P6094995 & L. Vautier & s.n. & 24 & 5 \\
\hline G & P6094980 & Charles Bélanger & s.n. & n.a. & n.a. \\
\hline G & P6094983 & Dr. Montagne & s.n. & n.a. & n.a. \\
\hline G & P6094972 & Chambion (?) & s.n. & n.a. & 6 \\
\hline G & P6094971 & Cadet & s.n. & n.a. & 6 \\
\hline G & P6095066 & Delessert & s.n. & n.a. & n.a. \\
\hline G & P6094979 & Garrot & s.n. & n.a. & n.a. \\
\hline G & P6094886 & Abbé Roquebrune & s.n. & n.a. & 7 \\
\hline G & P6094884 & Ph. Dunant & s.n. & n.a. & n.a. \\
\hline G & P6014878 & Boissier & s.n. & n.a. & n.a. \\
\hline G & P6014890 & leg. ign. & s.n. & n.a. & n.a. \\
\hline HUJ & HUJHERB 131645 & A. Eig & s.n. & 28 & 6 \\
\hline HUJ & HUJHERB 131640 & A. Eig & s.n. & 17 & 3 \\
\hline HUJ & HUJHERB 131644 & A. Danin & $72-14-23$ & 13 & 6 \\
\hline $\mathrm{K}$ & K001092999 & leg. ign. & 7 & 18 & 5 \\
\hline K & K001042848 & E. Serafino & 1487.1 & n.a. & 3 \\
\hline K & K001042835 & Liendon & s.n. & n.a. & 6 \\
\hline K & K001042840 & E. Bourgeau & 2 & 25 & 5 \\
\hline K & K001042809 & E. Reverchon & s.n. & $26 \& 6$ & $6 \& 8$ \\
\hline K & K001042836 & Canut & s.n. & 29 & 5 \\
\hline K & K001042801 & leg. ign. & s.n. & 3 & 4 \\
\hline K & K001042805 & J.M.H. Borel & s.n. & 10 & 6 \\
\hline K & K001042800 & leg. ign. & s.n. & 14 & 5 \\
\hline K & K001042815 & J.C. Joad & s.n. & 24 & 4 \\
\hline K & K001092994 & Ed. Bonnet & s.n. & 4 & 9 \\
\hline K & K001092996 & A. Cartier & s.n. & 20 & 5 \\
\hline K & K001092995 & Ed. Marçais & s.n. & n.a. & $6--7$ \\
\hline K & K001092997 & P. & s.n. & n.a. & 7 \\
\hline K & K001042806 & \multicolumn{2}{|c|}{ E. Ellman \& N.Y. Sandwy 99} & 6 & 6 \\
\hline K & K001042810 & J. Souster & 1288 & 15 & 6 \\
\hline K & K001264473 & leg. ign. & $144-70-01311$ & 26 & 8 \\
\hline K & K001042804 & F.N. Hepper & 8945 & 26 & 5 \\
\hline K & K001042881 & D.C. & s.n. & n.a. & n.a. \\
\hline K & K001042918 & Requien & s.n. & n.a. & n.a. \\
\hline K & K001264135 & M. Requien & s.n. & n.a. & n.a. \\
\hline K & K001042807 & Lombardelly & s.n. & n.a. & 6 \\
\hline
\end{tabular}




\begin{tabular}{|c|c|c|c|c|}
\hline K & K001042811 & leg. ign. & s.n. & n.a. \\
\hline K & K001042814 & J. Kutler (?) & s.n. & n.a. \\
\hline K & K001042808 & J.M.H. Borel & s.n. & n.a. \\
\hline MA & MA-01-00156308 & Mutel & s.n. & n.a. \\
\hline MA & MA-01-00038766 & P.F.A. Irat & 408 & 26 \\
\hline MA & MA-01-00038767 & F. Bruyas & 2629 & n.a. \\
\hline MA & MA-01-00038765 & Fre. Sennen & s.n. & 6 \\
\hline MA & MA-01-00471257 & L. Samat & s.n. & 25 \\
\hline MA & MA-01-00650348 & J.F. de Witte & 15027 & 19 \\
\hline MA & MA-01-00191210 & G. Gavelle & s.n. & 5 \\
\hline MA & MA-01-00290893 & G. Gavelle & s.n. & 11 \\
\hline MA & MA-01-00290896 & G. Gavelle & s.n. & 15 \\
\hline MA & MA-01-00650332 & J.F. de Witte & 15814 & 20 \\
\hline MA & MA-01-00650333 & J.F. de Witte & 16909 & 13 \\
\hline MA & MA-01-00650349 & J.F. de Witte & 16269 & 2 \\
\hline MA & MA-01-00204222 & \multicolumn{2}{|c|}{ Bellot, Casaseca \& Cast s.n. } & 25 \\
\hline MA & MA-01-00532346 & P. Bamps & 9002 & 12 \\
\hline MEL & MEL-2407544 & Viguier & S.n. & n.a. \\
\hline MEL & MEL-2319016 & John Stuart Mill & s.n. & n.a. \\
\hline MEL & MEL-2407545 & Canut & s.n. & 29 \\
\hline MEL & MEL-2407553 & Huguenin & s.n. & n.a. \\
\hline MEL & MEL-2407554 & leg. ign. & s.n. & n.a. \\
\hline MEL & MEL-2407555 & leg. ign. & s.n. & n.a. \\
\hline MEL & MEL-2407558 & Suffren & s.n. & n.a. \\
\hline MPU & n.a. & \multicolumn{2}{|c|}{ Herbier fac de médecine s.n. } & n.a. \\
\hline MPU & n.a. & Guillon & s.n. & 16 \\
\hline MPU & n.a. & \multicolumn{2}{|c|}{ Herbier fac de médecine s.n. } & 2 \\
\hline MPU & n.a. & Dunal & s.n. & 31 \\
\hline MPU & n.a. & Cambessèdes & s.n. & 17 \\
\hline MPU & n.a. & F. de Girard & s.n. & 25 \\
\hline MPU & n.a. & Guillon & s.n. & n.a. \\
\hline MPU & n.a. & A. Irat & s.n. & n.a. \\
\hline MPU & n.a. & E. Cosson & s.n. & 4 \\
\hline MPU & n.a. & L. Liendon & 391 a & n.a. \\
\hline MPU & n.a. & L. Liendon & $391 a$ & n.a. \\
\hline MPU & n.a. & Planchon & s.n. & n.a. \\
\hline MPU & n.a. & Liendon & 391 & n.a. \\
\hline MPU & n.a. & Guillon & s.n. & 2 \\
\hline MPU & n.a. & P.F.A. Irat & s.n. & n.a. \\
\hline MPU & n.a. & P.F.A. Irat & s.n. & 26 \\
\hline MPU & n.a. & P.F.A. Irat & 408 & 26 \\
\hline MPU & n.a. & E. Le Sourd-Dussisples & s.n. & n.a. \\
\hline MPU & n.a. & B. Mouly & 300 & n.a. \\
\hline MPU & n.a. & Hubert & s.n. & 21 \\
\hline
\end{tabular}




\begin{tabular}{|c|c|c|c|c|c|}
\hline MPU & n.a. & Dr Le Sourd & s.n. & 26 & 6 \\
\hline MPU & n.a. & Planchon & s.n. & n.a. & 6 \\
\hline MPU & n.a. & L. Pierre & s.n. & n.a. & n.a. \\
\hline MPU & n.a. & H. Hanry & s.n. & 6 & 5 \\
\hline MPU & n.a. & H. Hanry & 3003 & 6 & 5 \\
\hline MPU & n.a. & Canut & 391 & 29 & 5 \\
\hline MPU & n.a. & Dr Le Sourd & s.n. & 29 & 5 \\
\hline MPU & n.a. & Aubouy & s.n. & 3 & 6 \\
\hline MPU & n.a. & leg. ign. & s.n. & 20 & 5 \\
\hline MPU & n.a. & Herbier André & s.n. & n.a. & n.a. \\
\hline MPU & n.a. & Dr Le Sourd & s.n. & n.a. & 5 \\
\hline MPU & n.a. & Aubouy & s.n. & 18 & 5 \\
\hline MPU & n.a. & Aubouy & s.n. & 29 & 5 \\
\hline MPU & n.a. & Brunet & s.n. & 20 & 5 \\
\hline MPU & n.a. & Bernardin Martin & s.n. & n.a. & 6 \\
\hline MPU & n.a. & A. Tholin & s.n. & n.a. & 4 \\
\hline MPU & n.a. & Neyra & s.n. & 23 & 5 \\
\hline MPU & n.a. & A.B. & s.n. & 22 & 6 \\
\hline MPU & n.a. & Aubouy & s.n. & 4 & 7 \\
\hline MPU & n.a. & Reverchon & s.n. & $11 \& 18$ & $6 \& 8$ \\
\hline MPU & n.a. & A. Cartier & s.n. & 20 & 5 \\
\hline MPU & n.a. & A. Cartier & s.n. & 20 & 5 \\
\hline MPU & n.a. & J. Daveau & s.n. & n.a. & 5 \\
\hline MPU & n.a. & E. Reverchon & 340 & 14 & 6 \\
\hline MPU & n.a. & André & s.n. & n.a. & n.a. \\
\hline MPU & n.a. & Aubouy & s.n. & 30 & 6 \\
\hline MPU & n.a. & J.B. Barla & s.n. & 8 & 6 \\
\hline MPU & n.a. & E.J. Neyraut & s.n. & 29 & 5 \\
\hline MPU & n.a. & docteur E. Magnol & s.n. & n.a. & 6 \\
\hline MPU & n.a. & F. Mouret & s.n. & n.a. & 6 \\
\hline MPU & n.a. & E.J. Neyraut & 4794 & 3 & 6 \\
\hline MPU & n.a. & E.J. Neyraut & 4794 & 3 & 6 \\
\hline MPU & n.a. & E. Magnol & s.n. & 22 & 6 \\
\hline MPU & n.a. & Herbier C. Flahaut & s.n. & 14 & 6 \\
\hline MPU & n.a. & Aubouy & s.n. & 31 & 5 \\
\hline MPU & n.a. & Abbe A. Pons & s.n. & 12 & 6 \\
\hline MPU & n.a. & F. Bruyas & 2629 & n.a. & 6 \\
\hline MPU & n.a. & F. Bruyas & s.n. & n.a. & 6 \\
\hline MPU & n.a. & A. Guillon & s.n. & 7 & 7 \\
\hline MPU & n.a. & E.J. Neyraut & s.n. & 28 & 5 \\
\hline MPU & n.a. & C. Copineau & s.n. & 26 & 5 \\
\hline MPU & n.a. & Ecole St Paul & s.n. & 28 & 6 \\
\hline MPU & n.a. & Bertrand & 217 & 10 & 6 \\
\hline MPU & n.a. & Dr. Jouvence (?) & s.n. & n.a. & 5 \\
\hline
\end{tabular}




\begin{tabular}{|c|c|c|c|c|c|}
\hline MPU & n.a. & A. Raphélis & s.n. & n.a. & 5 \\
\hline MPU & n.a. & Faure & 120 & 3 & 6 \\
\hline MPU & n.a. & Faure & s.n. & 3 & 6 \\
\hline MPU & n.a. & A. Bourgeois & s.n. & n.a. & 7 \\
\hline MPU & n.a. & Fourès & s.n. & n.a. & 8 \\
\hline MPU & n.a. & Frère Sennen & s.n. & n.a. & n.a. \\
\hline MPU & n.a. & Frère Sennen & s.n. & n.a. & n.a. \\
\hline MPU & n.a. & Cassat & s.n. & 5 & 7 \\
\hline MPU & n.a. & Frère Sennen & s.n. & n.a. & n.a. \\
\hline MPU & n.a. & C.A. Gérard & 4674 & 12 & 6 \\
\hline MPU & n.a. & Frère Sennen & s.n. & n.a. & 5 \\
\hline MPU & n.a. & C.A. Gérard & 4674 & 12 & 6 \\
\hline MPU & n.a. & A. Guillon & s.n. & 30 & 6 \\
\hline MPU & n.a. & J. Stefani & s.n. & 10 & 6 \\
\hline MPU & n.a. & Fourès & s.n. & n.a. & 6 \\
\hline MPU & n.a. & E.J. Neyraut & s.n. & 30 & 5 \\
\hline MPU & n.a. & J. de Vichet & s.n. & 12 & 6 \\
\hline MPU & n.a. & J. de Vichet & s.n. & 28 & 5 \\
\hline MPU & n.a. & P. Cousturier & s.n. & n.a. & 4 \\
\hline MPU & n.a. & Albaille & s.n. & 15 & 4 \\
\hline MPU & n.a. & J.B. Renaud & s.n. & 5 & 6 \\
\hline MPU & n.a. & L. Samat & 6059 & 25 & 5 \\
\hline MPU & n.a. & E.J. Neyraut & s.n. & 14 & 10 \\
\hline MPU & n.a. & leg. ign. & s.n. & 12 & 6 \\
\hline MPU & n.a. & S.J. Albaille & s.n. & 13 & 6 \\
\hline MPU & n.a. & S.J. Albaille & s.n. & 13 & 6 \\
\hline MPU & n.a. & S.J. Albaille & s.n. & 10--20 & 6 \\
\hline MPU & n.a. & G. Gavelle & 4269 & 15 & 6 \\
\hline MPU & n.a. & G. Blanchet & s.n. & n.a. & 6 \\
\hline MPU & n.a. & G. Blanchet & s.n. & 12 & 6 \\
\hline MPU & n.a. & Santo & s.n. & 12 & 6 \\
\hline MPU & n.a. & E.J. Neyraut & s.n. & 29--1 & $5--7$ \\
\hline MPU & n.a. & Aucher (?) & 55 & n.a. & n.a. \\
\hline MPU & n.a. & Massot & s.n. & n.a. & n.a. \\
\hline MPU & n.a. & Herbier Albaille & s.n. & n.a. & n.a. \\
\hline MPU & n.a. & J.B. Renaud & 111 & 5 & 6 \\
\hline MPU & n.a. & S.J. Albaille & 301 & n.a. & n.a. \\
\hline MPU & n.a. & Abbé Gausserand & s.n. & n.a. & n.a. \\
\hline MPU & n.a. & Dr Le Sourd & s.n. & n.a. & n.a. \\
\hline MPU & n.a. & Lourbarelle (?) & s.n. & n.a. & n.a. \\
\hline MPU & n.a. & Raphélis & s.n. & n.a. & $6--7$ \\
\hline $\mathrm{P}$ & P02575135 & leg. ign. & 685 & n.a. & 7 \\
\hline$P$ & P03175036 & leg. ign. & 117 & 22 & 5 \\
\hline$P$ & P03174919 & Jacquemont & s.n. & n.a. & 5 \\
\hline
\end{tabular}




\begin{tabular}{|c|c|c|c|c|}
\hline$P$ & P03175003 & Leman & s.n. & n.a. \\
\hline$P$ & P03174952 & A.P. de Candolle & s.n. & n.a. \\
\hline$P$ & P02391519 & Maire & s.n. & n.a. \\
\hline$P$ & P02810831 & M. Emerie (?) & s.n. & n.a. \\
\hline$P$ & P03175035 & H. de la Perraudière & s.n. & n.a. \\
\hline$P$ & P03175014 & W. de Schoenefeld & s.n. & n.a. \\
\hline$P$ & P02574937 & J.M. Despreaux & s.n. & n.a. \\
\hline$P$ & P03174939 & M. Bentham & s.n. & n.a. \\
\hline$P$ & P02391570 & $\mathrm{R}$. & s.n. & n.a. \\
\hline$P$ & P02391570 & $\mathrm{R}$. & s.n. & n.a. \\
\hline$P$ & P02575181 & Dunal & s.n. & n.a. \\
\hline$P$ & P03174925 & Perreymond & s.n. & n.a. \\
\hline$P$ & P03174938 & A. Lenormand & s.n. & n.a. \\
\hline$P$ & P02699559 & Grémer & s.n. & 25 \\
\hline$P$ & P02391520 & [illegible] & s.n. & 17 \\
\hline$P$ & P02391512 & P.F.A. Irat & 753 & 15 \\
\hline$P$ & P03174931 & Née (?) & s.n. & n.a. \\
\hline$P$ & P03175006 & Callou (?) & s.n. & n.a. \\
\hline$P$ & P02575078 & A. de St Hilaire & s.n. & n.a. \\
\hline$P$ & P03174978 & P.F.A. Irat & s.n. & n.a. \\
\hline$P$ & P03174954 & A. de St Hilaire & s.n. & n.a. \\
\hline$P$ & P03174974 & Schler (?) & s.n. & n.a. \\
\hline$P$ & P02391534 & A. Hertz (?) & s.n. & 5 \\
\hline$P$ & P03174947 & A. de St Hilaire & s.n. & n.a. \\
\hline$P$ & P02575074 & Cavalier & s.n. & n.a. \\
\hline$P$ & P02575099 & A. de St Hilaire & s.n. & n.a. \\
\hline$P$ & P02575069 & Fée & s.n. & n.a. \\
\hline$P$ & P03175022 & Henrici Lartigue & s.n. & 15 \\
\hline$P$ & P02391570 & Auzende & s.n. & 4 \\
\hline$P$ & P02391570 & Auzende & s.n. & 4 \\
\hline$P$ & P02575075 & F. Clement & s.n. & n.a. \\
\hline$P$ & P02575186 & A.G. de Dives & 18 & 10 \\
\hline $\mathrm{P}$ & P03174965 & De Lort & s.n. & n.a. \\
\hline$P$ & P03174951 & Dr. Grenier & s.n. & n.a. \\
\hline$P$ & P03174979 & A. Leclerc & s.n. & n.a. \\
\hline$P$ & P02575080 & Boyé & 50 & n.a. \\
\hline$P$ & P02575073 & L. Kralik & 1849 & 4 \\
\hline$P$ & P03175011 & Liendon & s.n. & n.a. \\
\hline$P$ & P03174935 & Liendon & s.n. & n.a. \\
\hline$P$ & P02569728 & Liendon & $391 a$ & n.a. \\
\hline$P$ & P03175004 & E. Bourgeau & 2 & 25 \\
\hline $\mathrm{P}$ & P03175012 & E. Bourgeau & 2 & 25 \\
\hline$P$ & P03175022 & E. Bourgeau & 2 & 25 \\
\hline$P$ & P03175027 & E. Bourgeau & 2 & 25 \\
\hline
\end{tabular}




\begin{tabular}{|c|c|c|c|c|c|}
\hline$P$ & P02569980 & Irat P.F.A. & 408 & 26 & 5 \\
\hline$P$ & P02569654 & Irat P.F.A. & 408 & 26 & 5 \\
\hline$P$ & P02391587 & Irat P.F.A. & 408 & 26 & 5 \\
\hline$P$ & P02391513 & O. Debeaux & s.n. & n.a. & 6 \\
\hline$P$ & P02575079 & \multicolumn{2}{|c|}{ Maréchal de Bontarly (?) s.n. } & n.a. & 6 \\
\hline $\mathrm{P}$ & P03175018 & A. Guillon & s.n. & 11 & 6 \\
\hline$P$ & P03175029 & Leclerc & s.n. & n.a. & n.a. \\
\hline$P$ & P02530409 & Orth. André & s.n. & n.a. & 6 \\
\hline$P$ & P02575156 & E. Mouillefarine & 3 & n.a. & 7 \\
\hline$P$ & P02575180 & J. Lloyd & s.n. & n.a. & 6 \\
\hline$P$ & P03174969 & Ch. Gontartiez & s.n. & n.a. & $7--8$ \\
\hline$P$ & P02575209 & Ch. Pontarlier & s.n. & n.a. & 6 \\
\hline$P$ & P03174964 & Louis Amblard & s.n. & 21 & 6 \\
\hline$P$ & P02575065 & Loret & s.n. & n.a. & n.a. \\
\hline$P$ & P02575208 & \multicolumn{2}{|c|}{ Institut Botanique de Ca s.n. } & n.a. & 9 \\
\hline$P$ & P02575066 & leg. ign. & s.n. & n.a. & 8--9 \\
\hline$P$ & P02530404 & Orth. André & s.n. & 15 & 7 \\
\hline$P$ & P03174968 & Lepeltier & s.n. & n.a. & 7 \\
\hline $\mathrm{P}$ & P03175037 & Ripart & s.n. & n.a. & 8 \\
\hline$P$ & P03174967 & Eloy de Vicq & s.n. & 20 & 9 \\
\hline$P$ & P03174994 & J. Parseval & s.n. & 7 & 6 \\
\hline$P$ & P02569726 & Laguesse A. & s.n. & 17 & 6 \\
\hline$P$ & P02530407 & L'Abbé Lelièvre & s.n. & 27 & 6 \\
\hline$P$ & P03175031 & leg. ign. & s.n. & 27 & 5 \\
\hline$P$ & P03174928 & C. Billot & $3003\left(1868 n^{\circ} 6\right)$ & n.a. & $5--6$ \\
\hline$P$ & P03175023 & C. Billot & 3003 & n.a. & $5--6$ \\
\hline$P$ & P03175005 & C. Billot & 3003 & n.a. & $5--6$ \\
\hline$P$ & P03174977 & Ch. Ozanon & s.n. & n.a. & 6 \\
\hline $\mathrm{P}$ & P03174963 & Ch. Ozanon & s.n. & n.a. & 6 \\
\hline$P$ & P03174926 & G. Thuret & 61 & 20 & 6 \\
\hline$P$ & P03175034 & H. de la Perraudière & s.n. & 27 & 6 \\
\hline $\mathrm{P}$ & P02575100 & H. Hanry & 3003 & 6 & 5 \\
\hline$P$ & P02575068 & H. Hanry & 3003 & 6 & 5 \\
\hline$P$ & P02575160 & H. Hanry & 3003 & 6 & 5 \\
\hline$P$ & P02530405 & L'Abbé Lelièvre & s.n. & 3 & 6 \\
\hline$P$ & P02575210 & D.M. Roux & s.n. & n.a. & 6 \\
\hline$P$ & P03175002 & [illegible] & s.n. & n.a. & 4 \\
\hline$P$ & P02570013 & E. Bourgeau & s.n. & 17 & 5 \\
\hline$P$ & P03175024 & E. Bourgeau & s.n. & 27 & 5 \\
\hline$P$ & P03175030 & E. Bourgeau & s.n. & 27 & 5 \\
\hline$P$ & P03174944 & E. Bourgeau & s.n. & 27 & 5 \\
\hline $\mathrm{P}$ & P03174994 & E. Bourgeau & s.n. & 27 & 5 \\
\hline$P$ & P02570012 & Canat & s.n. & 29 & 5 \\
\hline$P$ & P03174945 & Canut & s.n. & 29 & 5 \\
\hline
\end{tabular}




\begin{tabular}{|c|c|c|c|c|}
\hline$P$ & P03175028 & Canut & s.n. & 29 \\
\hline$P$ & P03175020 & Canut & s.n. & 29 \\
\hline$P$ & P03174995 & Canut & s.n. & 29 \\
\hline$P$ & P02569978 & Canut & 391 & 29 \\
\hline$P$ & P03174953 & Dr Lebel & s.n. & n.a. \\
\hline $\mathrm{P}$ & P00799039 & Canut & 391 & 29 \\
\hline$P$ & P00685973 & A. Legrand & s.n. & 1 \\
\hline$P$ & P03174962 & Dr Bras & s.n. & 21 \\
\hline$P$ & P03175017 & Bocquillon & s.n. & n.a. \\
\hline$P$ & P02569725 & Th. Delacour & s.n. & n.a. \\
\hline$P$ & P02575067 & E.M. Sauvage & s.n. & 27 \\
\hline$P$ & P03174923 & Marcilly & s.n. & 29 \\
\hline$P$ & P03174927 & Bureau & s.n. & 22 \\
\hline$P$ & P03175038 & Lefèvre & s.n. & 3 \\
\hline$P$ & P03175001 & A. Franchet & s.n. & n.a. \\
\hline$P$ & P03174943 & leg. ign. & s.n. & 12 \\
\hline$P$ & P02569729 & Th. Delacour & s.n. & 26 \\
\hline$P$ & P02570028 & Grandclément & s.n. & n.a. \\
\hline$P$ & P02569657 & C. Damiens & s.n. & $4--5$ \\
\hline$P$ & P02391605 & H. van Heurck & s.n. & n.a. \\
\hline$P$ & P02569716 & Eugène & s.n. & 15 \\
\hline$P$ & P03174997 & O. Debeaux & s.n. & 6 \\
\hline$P$ & P02305581 & Warnier & 22 & n.a. \\
\hline$P$ & P02569999 & X. Vendrely & s.n. & 16 \\
\hline$P$ & P02575211 & Chauvin & s.n. & n.a. \\
\hline$P$ & P02569998 & H. Hervier-Basson & s.n. & 27 \\
\hline$P$ & P02570065 & leg. ign. & s.n. & 29 \\
\hline$P$ & P02570076 & Société de Champagne & s.n. & n.a. \\
\hline $\mathrm{P}$ & P02570009 & Th. Delacour & s.n. & 27 \\
\hline$P$ & P02570001 & Ch. Pontarlier & s.n. & 4 \\
\hline$P$ & P03175033 & M. Franchet & s.n. & n.a. \\
\hline$P$ & P02569656 & Th. Delacour & s.n. & $1--2$ \\
\hline$P$ & P05591715 & leg. ign. & s.n. & n.a. \\
\hline$P$ & P02569993 & $H($ ?)aures & s.n. & n.a. \\
\hline$P$ & P03174949 & J. Daneau & s.n. & n.a. \\
\hline$P$ & P02569979 & L. Anthouard & s.n. & 1 \\
\hline$P$ & P03174940 & leg. ign. & s.n. & n.a. \\
\hline$P$ & P02530406 & R.B. & s.n. & 4 \\
\hline$P$ & P03174993 & A. Cartier & s.n. & n.a. \\
\hline$P$ & P02570004 & J. Gautier-Lacroze & s.n. & n.a. \\
\hline$P$ & P02391550 & A. Cartier & 1487 & 20 \\
\hline $\mathrm{P}$ & P03174929 & A. Cartier & 1487 & 20 \\
\hline$P$ & P02569995 & A. Cartier & 1487 & 20 \\
\hline$P$ & P02570014 & A. Cartier & 1487 & 20 \\
\hline
\end{tabular}




\begin{tabular}{|c|c|c|c|c|c|}
\hline$P$ & P03174990 & E. Reverchon & s.n. & 27 & 6 \\
\hline$P$ & P03174987 & Ed. Marçais & s.n. & n.a. & 8 \\
\hline$P$ & P02575169 & J. Daveau & s.n. & 28 & 5 \\
\hline$P$ & P02575207 & E. Marçay & s.n. & n.a. & $6--7$ \\
\hline$P$ & P02569994 & E. Marçay & s.n. & n.a. & $6--7$ \\
\hline$P$ & P02570005 & E. Marçay & s.n. & n.a. & $6--7$ \\
\hline$P$ & P02391524 & leg. ign. & s.n. & 20 & 7 \\
\hline$P$ & P03174988 & \multicolumn{2}{|c|}{ V. Retalon (or Metalon?) s.n. } & 20 & 8 \\
\hline$P$ & P03174933 & E. Reverchon & 340 & 14 & 6 \\
\hline$P$ & P03175009 & E. Reverchon & 340 & 14 & 6 \\
\hline$P$ & P03174973 & E. Reverchon & 340 & 14 & 6 \\
\hline$P$ & P02569727 & E. Reverchon & 340 & 14 & 6 \\
\hline$P$ & P02570113 & J.B. Barla & s.n. & n.a. & 6 \\
\hline$P$ & P02391544 & C. Ozanon & s.n. & 25 & 8 \\
\hline$P$ & P02570000 & C. Ozanon & s.n. & 25 & 8 \\
\hline$P$ & P03174976 & leg. ign. & s.n. & 12 & 9 \\
\hline$P$ & P03175000 & Flahaut & s.n. & n.a. & n.a. \\
\hline$P$ & P02569655 & Th. Delacour & s.n. & 10 & 6 \\
\hline$P$ & P03174934 & E. Reverchon & s.n. & 14 & 6 \\
\hline$P$ & P03174992 & E. Reverchon & s.n. & 19 & 6 \\
\hline$P$ & P03174946 & E. Reverchon & s.n. & n.a. & n.a. \\
\hline$P$ & P02570104 & leg. ign. & s.n. & 6 & 7 \\
\hline$P$ & P02391577 & Ch. Ozanon, Dr. Gillot & s.n. & 22 & 7 \\
\hline$P$ & P03175026 & Ch. Ozanon, Dr. Gillot & s.n. & 22 & 7 \\
\hline$P$ & P03156804 & Ch. Ozanon, Dr. Gillot & s.n. & 22 & 7 \\
\hline$P$ & P03174986 & L. Chevallier & s.n. & 22 & 6 \\
\hline$P$ & P03175021 & V. Auzat & s.n. & 28 & 7 \\
\hline$P$ & P02575182 & E. Jeanpert & s.n. & n.a. & 9 \\
\hline$P$ & P02570067 & C. Copineau & s.n. & 15 & 6 \\
\hline$P$ & P02570008 & F. Bruyas & 2629 & n.a. & 6 \\
\hline$P$ & P03174991 & F. Bruyas & 2629 & n.a. & 6 \\
\hline$P$ & P03174998 & F. Bruyas & 2629 & n.a. & 6 \\
\hline$P$ & P03174941 & F. Bruyas & 2629 & n.a. & 6 \\
\hline$P$ & P02391521 & Hubert & s.n. & 21 & 6 \\
\hline$P$ & P02575185 & J.B. Barla & s.n. & 30 & 5 \\
\hline$P$ & P02570027 & X. Vendrely & s.n. & n.a. & n.a. \\
\hline$P$ & P02575204 & E. Dupuy & s.n. & n.a. & 6 \\
\hline$P$ & P02575184 & J.B. Barla & s.n. & 23 & 5 \\
\hline$P$ & P02570006 & L. Conill & s.n. & 18 & 5 \\
\hline$P$ & P02575096 & Ch. Hermant & s.n. & n.a. & $6--7$ \\
\hline$P$ & P02575206 & S. Savouré & $562 c$ & 7 & 6 \\
\hline$P$ & P02575219 & J. Bozon & s.n. & 12 & 6 \\
\hline$P$ & P03174980 & R. Rotgès & s.n. & 28 & 5 \\
\hline$P$ & P02575183 & Cassat & s.n. & 5 & 7 \\
\hline
\end{tabular}




\begin{tabular}{|c|c|c|c|c|c|}
\hline $\mathrm{P}$ & P02570098 & Fre Sennen & s.n. & n.a. & n.a. \\
\hline$P$ & P03175040 & H. Coste & s.n. & 13 & 7 \\
\hline$P$ & P02575205 & S. Savouré & 695 & 16 & 11 \\
\hline$P$ & P02569976 & A. Cassat & s.n. & 18 & 7 \\
\hline$P$ & P03175015 & Bécon & 312 & 22 & 7 \\
\hline$P$ & P02570111 & H. Coste & 323 & n.a. & 7 \\
\hline$P$ & P03174996 & C.-A. Gérard & 4674 & 12 & 6 \\
\hline$P$ & P03175007 & C.-A. Gérard & 4674 & 12 & 6 \\
\hline$P$ & P02570099 & Stéfani & 4674 & 12 & 6 \\
\hline$P$ & P02575188 & J. Strici & s.n. & 29 & 4 \\
\hline$P$ & P02575187 & Ciprillon & s.n. & 30 & 6 \\
\hline$P$ & P03175025 & Hoschedé & s.n. & 17 & 6 \\
\hline$P$ & P02569730 & Sennen & s.n. & n.a. & 5 \\
\hline$P$ & P02575189 & E. Coraze & s.n. & 25 & 6 \\
\hline$P$ & P00620192 & Legris & s.n. & 3 & 7 \\
\hline$P$ & P04560803 & Legris & s.n. & 3 & 7 \\
\hline$P$ & P02523825 & Ch. Guffroy & 111 & n.a. & n.a. \\
\hline$P$ & P03174950 & G. Hibon & 111 & n.a. & n.a. \\
\hline$P$ & P03174942 & G. Hibon & 111 & n.a. & 6 \\
\hline$P$ & P02575157 & W.R. Fontan & s.n. & 15 & 6 \\
\hline$P$ & P02570101 & Port & s.n. & 13 & 8 \\
\hline$P$ & P02570102 & Port & s.n. & 13 & 8 \\
\hline$P$ & P02570110 & L. Bee & 321 & n.a. & 6 \\
\hline $\mathrm{P}$ & P03175013 & Dr. Lermoyez & 93 & n.a. & n.a. \\
\hline$P$ & P02575137 & J. de Vichet & s.n. & n.a. & 6 \\
\hline$P$ & P02575095 & Ch. Guffroy & s.n. & n.a. & n.a. \\
\hline$P$ & P03174982 & A. et E.G. Camus & s.n. & 19 & 6 \\
\hline$P$ & P02575098 & Ch. Guffroy & s.n. & 19 & 6 \\
\hline$P$ & P03174948 & Humbert & s.n. & 6 & 6 \\
\hline$P$ & P03174983 & A. et E.G. Camus & s.n. & 2 & 7 \\
\hline$P$ & P03174959 & Camus & s.n. & 11 & 6 \\
\hline$P$ & P03174984 & E.G. Camus & s.n. & n.a. & 6 \\
\hline $\mathrm{P}$ & P03174966 & L'abbé Somers & s.n. & 15 & 1 \\
\hline$P$ & P02575167 & J.J. Weber & s.n. & 2 & 6 \\
\hline$P$ & P02575164 & J.J. Weber & s.n. & 29 & 5 \\
\hline$P$ & P02575077 & J.B. Renaud & s.n. & n.a. & $5--6$ \\
\hline$P$ & P02575101 & J.B. Renaud & s.n. & n.a. & $5--6$ \\
\hline$P$ & P02559213 & Jean Guillet & 93 & 29 & 7 \\
\hline$P$ & P03174985 & A. Camus & s.n. & n.a. & 10 \\
\hline$P$ & P03175016 & L. Rodriguez & s.n. & 7 & 9 \\
\hline$P$ & P02570112 & J. Arènes & 324 & n.a. & 5 \\
\hline$P$ & P03174961 & M. Hickel & s.n. & n.a. & 6 \\
\hline$P$ & P02569977 & L. Giraudias & s.n. & n.a. & 7 \\
\hline$P$ & P02575165 & Melle Humbert & s.n. & n.a. & 5 \\
\hline
\end{tabular}




\begin{tabular}{|c|c|c|c|c|c|}
\hline P & P02570066 & leg. ign. & s.n. & 6 & 5 \\
\hline$P$ & P03174958 & \multicolumn{2}{|c|}{ Mariane de Gougerot-Ni s.n. } & n.a. & n.a \\
\hline$P$ & P02575166 & J. Arbost & s.n. & 15 & 6 \\
\hline$P$ & P03174956 & \multicolumn{2}{|c|}{ Mariane de Gougerot-Ni s.n. } & n.a. & 7 \\
\hline$P$ & P02575146 & B. de Retz & 273 & 20 & 7 \\
\hline$P$ & P02575144 & B. de Retz & 284 & 21 & 7 \\
\hline$P$ & P03174957 & \multicolumn{2}{|c|}{ Mariane de Gougerot-Ni s.n. } & n.a. & n.a \\
\hline$P$ & P03174955 & \multicolumn{2}{|c|}{ Mariane de Gougerot-Ni s.n. } & $\mathrm{v}$ & 6 \\
\hline$P$ & P02575138 & L. Samat & 6059 & 25 & 5 \\
\hline$P$ & P02575139 & L. Samat & 273 bis & 25 & 5 \\
\hline$P$ & P02570109 & L. Samat & 322 & 25 & 5 \\
\hline$P$ & P02559215 & CL. Legros & s.n. & 5 & 6 \\
\hline$P$ & P02575178 & R. L'Hermite & s.n. & n.a. & 6 \\
\hline$P$ & P02575136 & B. de Retz & 273 ter & 14 & 7 \\
\hline$P$ & P03187614 & E. Delpature & s.n. & n.a. & 6 \\
\hline$P$ & P02575162 & M. Homolle & s.n. & 26 & 6 \\
\hline$P$ & P02575178 & R. L'Hermite & s.n. & 3 & 6 \\
\hline$P$ & P04560804 & L. Rotereau & s.n. & n.a. & 6 \\
\hline$P$ & P02575168 & Mme Haffner & s.n. & 17 & 7 \\
\hline$P$ & P02575142 & B. de Retz & 14753 & 7 & 6 \\
\hline$P$ & P02575163 & Mme Haffner & s.n. & 14 & 8 \\
\hline$P$ & P03174981 & Dr. Defrance & s.n. & n.a. & 6 \\
\hline$P$ & P02575072 & Bournerias & 1534 & n.a. & 6 \\
\hline$P$ & P00731924 & E. Licent & s.n. & 3 & 6 \\
\hline$P$ & P02559210 & Henri Bouby & 860 & n.a. & 8 \\
\hline$P$ & P03187615 & E. Delpature & s.n. & n.a. & 6 \\
\hline$P$ & P02575143 & B. de Retz & 23558 & 2 & 6 \\
\hline$P$ & P02593382 & Paul Jovet & s.n. & 2 & 6 \\
\hline$P$ & P02559212 & Henri Bouby & 10.017 & 6 & 6 \\
\hline$P$ & P02559217 & J. Raynal & 360 & n.a. & 7 \\
\hline$P$ & P02570100 & leg. ign. & s.n. & 17 & 6 \\
\hline$P$ & P02569701 & H. Gillet & s.n. & 15 & 5 \\
\hline$P$ & P02575159 & B. de Retz & 39707 & 10 & 6 \\
\hline$P$ & P02575158 & B. de Retz & 39603 & 9 & 6 \\
\hline$P$ & P02559214 & J. Charrier & s.n. & 19 & 6 \\
\hline$P$ & P02575097 & J. Charrier & 3363 & 30 & 6 \\
\hline$P$ & P02575140 & J. Charrier & 3363 & 30 & 6 \\
\hline$P$ & P02575147 & J. Charrier & 3363 & 30 & 6 \\
\hline$P$ & P02559216 & J. Raynal & 2966 & 13 & 6 \\
\hline$P$ & P01969279 & Paul Jovet & s.n. & 7 & 9 \\
\hline$P$ & P03024565 & Jacqueline Tatigne & s.n. & 22 & 6 \\
\hline$P$ & P00056285 & P. Jovet & s.n. & 26 & 6 \\
\hline$P$ & P02575070 & E. Contré & 4268 & 22 & 6 \\
\hline$P$ & P02575161 & E. Contré & 4268 & 22 & 6 \\
\hline
\end{tabular}




\begin{tabular}{|c|c|c|c|c|}
\hline$P$ & P02575071 & Gavelle G. & 4269 & 15 \\
\hline$P$ & P02575145 & Gavelle G. & 4269 & 15 \\
\hline$P$ & P01969527 & Paul Jovet & s.n. & 21 \\
\hline$P$ & P02589843 & Paul Jovet & s.n. & 13 \\
\hline$P$ & P02559211 & Henri Bouby & 10.000 & 27 \\
\hline$P$ & P03618090 & \multicolumn{2}{|c|}{ G.G. Aymonin et Moniqı 28281} & 6 \\
\hline$P$ & P03618089 & \multicolumn{2}{|c|}{ G.G. Aymonin et Moniqu 28271} & 6 \\
\hline$P$ & P03618088 & \multicolumn{2}{|c|}{ G.G. Aymonin et Moniqu 29529} & $6--7$ \\
\hline$P$ & P02582389 & F.N. Hepper & 8945 & 26 \\
\hline$P$ & P02575141 & Bamps & 9002 & 12 \\
\hline$P$ & P00247257 & Anaclet & s.n. & n.a. \\
\hline$P$ & P04560807 & Viel-Cazal & s.n. & n.a. \\
\hline$P$ & P00836295 & \multicolumn{2}{|c|}{ F. Jabbour, M. Le Guillou 9} & 6 \\
\hline$P$ & P00836297 & \multicolumn{2}{|c|}{ F. Jabbour, M. Le Guillou 7} & 6 \\
\hline$P$ & P00836301 & \multicolumn{2}{|c|}{ F. Jabbour, M. Le Guillou 3} & 6 \\
\hline$P$ & P00836302 & \multicolumn{2}{|c|}{ F. Jabbour, M. Le Guillou 2} & 6 \\
\hline$P$ & P00836303 & \multicolumn{2}{|c|}{ F. Jabbour, M. Le Guillou 1} & 6 \\
\hline$P$ & P00836296 & \multicolumn{2}{|c|}{ F. Jabbour, M. Le Guillou 8} & 6 \\
\hline$P$ & P00836298 & \multicolumn{2}{|c|}{ F. Jabbour, M. Le Guillou 6} & 6 \\
\hline$P$ & P00836299 & \multicolumn{2}{|c|}{ F. Jabbour, M. Le Guillou 5} & 6 \\
\hline$P$ & P00836300 & \multicolumn{2}{|c|}{ F. Jabbour, M. Le Guillou 4} & 6 \\
\hline$P$ & P03175010 & l'Abbé Pourret & s.n. & n.a. \\
\hline$P$ & P03174972 & Requien & s.n. & n.a. \\
\hline$P$ & P03174960 & H. dul. (?) & s.n. & n.a. \\
\hline$P$ & P03175019 & [illegible] & s.n. & n.a. \\
\hline$P$ & P02391566 & Chaubard & s.n. & n.a. \\
\hline$P$ & P02570003 & leg. ign. & s.n. & n.a. \\
\hline$P$ & P03174720 & l'Abbé Pourret & s.n. & n.a. \\
\hline$P$ & P03175005 & A. Gorvioy (?) & s.n. & n.a. \\
\hline$P$ & P02530408 & Dr. Miergues (Herbier) & s.n. & n.a. \\
\hline$P$ & P03175008 & E. Reverchon & s.n. & 14 \\
\hline$P$ & P03174999 & \multicolumn{2}{|c|}{ Ex herb. Marchnis d'A. d s.n. } & n.a. \\
\hline$P$ & P03174936 & Giort (?) & s.n. & n.a. \\
\hline$P$ & P02391535 & Lamarlière (?) & s.n. & n.a. \\
\hline$P$ & PL02313637 & Legris & s.n. & n.a. \\
\hline$P$ & P02391587 & Liendon & 391 & n.a. \\
\hline$P$ & P03175032 & Louis Hurot & s.n. & n.a. \\
\hline$P$ & P03174970 & leg. ign. & s.n. & n.a. \\
\hline$P$ & P03174989 & leg. ign. & s.n. & n.a. \\
\hline$P$ & P03175005 & Requien & s.n. & n.a. \\
\hline$P$ & P03174930 & A. Franchet & s.n. & n.a. \\
\hline$P$ & P02575154 & Berthoumieu & s.n. & n.a. \\
\hline$P$ & P03262078 & O.B. & $8 / 293$ & n.a. \\
\hline$P$ & P02575076 & Sarmentier & s.n. & n.a. \\
\hline
\end{tabular}




\begin{tabular}{|c|c|c|c|c|c|}
\hline $\mathrm{P}$ & P02874246 & Dumont D'Urville & s.n. & n.a. & n.a. \\
\hline$P$ & P02575179 & leg. ign. & $111(1534)$ & n.a. & n.a. \\
\hline PAL & PAL-Gr 100355 & Dr. Antoine Bras & s.n. & 21 & 7 \\
\hline PAL & PAL-Gr 100354 & Dr. Antoine Bras & 39 & 27 & 7 \\
\hline S & S16-2583 & Reverchon & s.n. & 14 & 6 \\
\hline$S$ & S16-2850 & Ch. Pont $[\ldots]$ & s.n. & 1 & 7 \\
\hline$S$ & S16-2827 & Carl de Geer & s.n. & 8 & 4 \\
\hline$S$ & S16-2600 & F. Bruyas & 2629 & n.a. & 6 \\
\hline$S$ & S16-2603 & Gombault & s.n. & 7 & 6 \\
\hline$S$ & S16-2584 & C. Bicknell & s.n. & 4 & 5 \\
\hline$S$ & S14-14265 & Hj. \& A. Hafstroem & s.n. & 18 & 7 \\
\hline$S$ & S16-2912 & Hj. Hafstroem & s.n. & 18 & 7 \\
\hline$S$ & S16-2594 & Irat & 408 & n.a. & 5 \\
\hline$S$ & S16-2867 & Petit & s.n. & n.a. & n.a. \\
\hline TO & dscn5665 & A. Goiran & s.n. & n.a. & n.a. \\
\hline TO & dscn5682 & [illegible] & s.n. & 17 & 6 \\
\hline TO & dscn5647 & Baraize & s.n. & n.a. & 6 \\
\hline TO & dscn5653 & J.B. Barla & s.n. & 23 & 5 \\
\hline TO & dscn5660 & J. Stefani & s.n. & 10 & 6 \\
\hline UPS & V-738373 & \multicolumn{2}{|c|}{ Meddelad af S.N. Casstris.n. } & n.a. & n.a. \\
\hline UPS & V-738026 & A. de Saint-Hilaire & s.n. & n.a. & 5 \\
\hline UPS & V-738322 & Smet[...] (?) & s.n. & n.a. & 5 \\
\hline UPS & V-738323 & A. Théveneau & s.n. & 5 & 6 \\
\hline UPS & V-738025 & Alphonse Autheman & s.n. & n.a. & 6 \\
\hline UPS & V-737956 & Ch. Fontarlier & s.n. & 10 & 7 \\
\hline UPS & V-738372 & Carl de Geer & s.n. & n.a. & 5 \\
\hline UPS & V-738355 & Elisée Reverchon & s.n. & 20 & 5 \\
\hline UPS & V-738006 & Carl de Geer & s.n. & 8 & 4 \\
\hline UPS & V-738020 & leg. ign. & s.n. & n.a. & n.a. \\
\hline W & $2008-21618$ & Irat & 408 & 26 & 5 \\
\hline W & 17906 & P.F.A. Irat & 408 & 26 & 5 \\
\hline W & 14219 & P.F.A. Irat & 408 & 26 & 5 \\
\hline W & $1996-11639$ & A. Tillet & s.n. & n.a. & 7 \\
\hline W & $1994-04540$ & Ch. Tontarliev & 7104 & 15 & 7 \\
\hline W & $1996-11638$ & Cartier & s.n. & n.a. & 5 \\
\hline W & 16409 & Marnac & 6 & 19 & 6 \\
\hline W & 1999-01546 & \multicolumn{2}{|c|}{ K.H. Rechinger \& H. Sleu 1628} & $16--2$ & $6--7$ \\
\hline W & 26236 & Retz & 23558 & 2 & 6 \\
\hline W & $2002-05181$ & F. Krendl & s.n. & 5 & 7 \\
\hline W & 2000-13105 & Froebe & 7832 & 21 & 5 \\
\hline
\end{tabular}




\begin{tabular}{|c|c|c|c|c|}
\hline late & Country & Collection locality & \multirow[t]{2}{*}{ Other notes c } & Cultivated? \\
\hline Year & & & & \\
\hline 1849 & France & \multicolumn{2}{|c|}{ Champs et vignes prés Auch (Ger.n.a. } & No \\
\hline 1849 & France & \multicolumn{2}{|c|}{ Champs et vignes prés Auch (Ger.n.a. } & No \\
\hline 1862 & France & \multirow{3}{*}{$\begin{array}{l}\text { Coteaux du Vinaigrié près Nice. } \\
\text { Coteaux du Vinaigrié près Nice. } \\
\text { [illegible] }\end{array}$} & \multirow{3}{*}{$\begin{array}{l}\text { Relighiae Mai } \\
\text { n.a. }\end{array}$} & No \\
\hline 1862 & France & & & No \\
\hline 1866 & France & & & No \\
\hline 1885 & France & \multirow{2}{*}{$\begin{array}{l}\text { Saargebiet, Louisenthal } \\
\text { champs Montpellier }\end{array}$} & $200 \mathrm{~m}$, in Gar & Yes \\
\hline 1896 & France & & Relighiae Mai & No \\
\hline 1907 & France & \multicolumn{2}{|c|}{ Hagenau, Wegersheim-bei einem n.a. } & Yes \\
\hline 1910 & France & \multicolumn{2}{|c|}{ Strassburg, Neudorf alter (...?) plena } & No \\
\hline 1916 & France & \multicolumn{2}{|l|}{ Montrieux: wenbergs-Terras? } & No \\
\hline 1916 & France & \multicolumn{2}{|c|}{ Montrieux: wenbergs-Terras? n.a. } & No \\
\hline 1991 & France & \multicolumn{2}{|c|}{ Drome, Mollons-sur-Ouvèze, anci 270 m, flanc s } & No \\
\hline 1865 & France & cambes (gir) & Dans les haie: & No \\
\hline 1879 & France & \multicolumn{2}{|c|}{ Coteaux de Landau, près Bourg-Sın.a. } & No \\
\hline 1889 & France & Bois de Balma & n.a. & No \\
\hline 1899 & France & aude: la nouvelle & Friche & No \\
\hline 1901 & France & Corse: Bonifacio & sous les olivi $\epsilon$ & No \\
\hline 1901 & France & Corse: Bonifacio & sous les olivi $\epsilon$ & No \\
\hline 1911 & France & Bouches-du-Rhone: luynes & champs et lie & No \\
\hline 1916 & France & Rians & Bord des chel & No \\
\hline 1930 & France & \multicolumn{2}{|c|}{ Bouches-du-Rhone: La Valentine. n.a. } & No \\
\hline 1963 & France & Grasse & Talus, fossés & No \\
\hline 1980 & France & Lyon & n.a. & No \\
\hline 1991 & France & \multicolumn{2}{|c|}{ Mollans-sur-Ouvèze (Drome), anc 270 m, flanc } & No \\
\hline n.a. & France & Hyeres near Gapeau River & n.a. & No \\
\hline 1848 & France & Env. de Toulon: Fort Rouge & n.a. & No \\
\hline 1848 & France & Saintes, Charente Inf. & n.a. & No \\
\hline 1849 & France & Env. de Toulouse & n.a. & No \\
\hline 1854 & France & Rogliano & n.a. & No \\
\hline 1859 & France & Mt. Pharon, près Toulon & n.a. & No \\
\hline 1860 & France & Roquefavour & n.a. & No \\
\hline 1860 & France & Luc & Bords des chí & No \\
\hline 1864 & France & St. Canat (Bouches-du- Rhône) & n.a. & No \\
\hline 1867 & France & \multicolumn{2}{|c|}{ Moissons a Soubise (Charente-Inf n.a. } & No \\
\hline 1867 & France & Bastia, Insel Corsika & Früchte & No \\
\hline 1869 & France & Bastia & Collium incult & No \\
\hline 1874 & France & \multicolumn{2}{|c|}{ Environs de Costebelle, au dessus Terrain calcai } & No \\
\hline 1875 & France & \multirow{2}{*}{$\begin{array}{l}\text { [illegible] près de Gorniès } \\
\text { Le Luc }\end{array}$} & Moissons de & No \\
\hline 1877 & France & & bords de chai & No \\
\hline 1878 & France & \multicolumn{2}{|c|}{ Beaupuy (près Toulouse), alt. 200 n.a. } & No \\
\hline 1880 & France & Canetto près Bonifacio & Lieux incultes & No \\
\hline 1883 & France & Hyères & n.a. & No \\
\hline
\end{tabular}




\begin{tabular}{|c|c|c|c|}
\hline 1884 & France & near Rochelle & Cornfield we€ \\
\hline 1884 & France & Char. Infer. & n.a. \\
\hline 1889 & France & \multicolumn{2}{|c|}{ de la Rochelle a la Palisse (Chte In n.a. } \\
\hline 1891 & France & Magnil près Luçon (Vendée) & n.a. \\
\hline 1893 & France & \multicolumn{2}{|c|}{ Collines de la Gardiole, prèrs Mirєn.a. } \\
\hline 1894 & France & Environs de Nice & n.a. \\
\hline 1898 & France & Béziers: Pradines le Haut & n.a. \\
\hline 1911 & France & \multicolumn{2}{|c|}{ Mt Agel, S.E Face above Grand Cc n.a. } \\
\hline 1925 & France & Pont de la Fou & n.a. \\
\hline 1930 & France & \multicolumn{2}{|c|}{ La Valentine: Friche à Mont-Riant n.a. } \\
\hline 1930 & France & \multicolumn{2}{|c|}{ La Valentine, Friche a Mont-Riant n.a. } \\
\hline 1991 & France & \multicolumn{2}{|c|}{ Mollans-sur-Ouvèze (Drôme): anc Flanc sud du 1} \\
\hline n.a. & France & Perpignan, py. or. & n.a. \\
\hline n.a. & France & Narbonne & n.a. \\
\hline 1849 & France & près Auch (Ger.) & Champs et vi६ \\
\hline 1825 & France & Avignon & n.a. \\
\hline 1825 & France & Narbonne & n.a. \\
\hline 1825 & France & Narbonne & n.a. \\
\hline 1825 & France & Fontfroid, Pyrénées Orientales & n.a. \\
\hline 1839 & France & Perpignan, Pyrénées Orientales & n.a. \\
\hline 1839 & France & Perpignan & n.a. \\
\hline 1839 & France & Toulon & n.a. \\
\hline 1855 & France & Brousse & n.a. \\
\hline 1862 & France & Herault, prope Valergues & n.a. \\
\hline 1867 & France & Bastia, Corse & n.a. \\
\hline 1867 & France & Le Luc, Var & n.a. \\
\hline 1880 & France & \multicolumn{2}{|c|}{ Corsica, Bonifacio, maquis de Can n.a. } \\
\hline 1880 & France & Bonifacio, Canetto & n.a. \\
\hline 1886 & France & Alpes Maritimes, Fontan & Moissons \\
\hline 1886 & France & Fontan, Alpes Maritimes & Moissons \\
\hline 1886 & France & Alpes Maritimes, Fontan & Moissons \\
\hline ante 1884 & France & Montpellier & n.a. \\
\hline ante 1888 & France & near Nice & n.a. \\
\hline n.a. & France & Lourmarin, Vaucluse & n.a. \\
\hline 1843 & France & \multicolumn{2}{|c|}{ Nice, La grotte d'amour, sur le Mın.a. } \\
\hline 1843 & France & Nice & n.a. \\
\hline 1845 & France & [...] circa Puy [...] (Gers) & n.a. \\
\hline 1849 & France & champs et vignes près Auch & n.a. \\
\hline 1851 & France & Corsica & n.a. \\
\hline 1856 & France & Vendée: Luçon & n.a. \\
\hline 1869 & France & Nizza & n.a. \\
\hline 1870 & France & Crest (Drôme) & n.a. \\
\hline 1870 & France & Crest (Drôme), Morillons & n.a. \\
\hline 1877 & France & Le luc (Var) bods des champs & n.a. \\
\hline
\end{tabular}




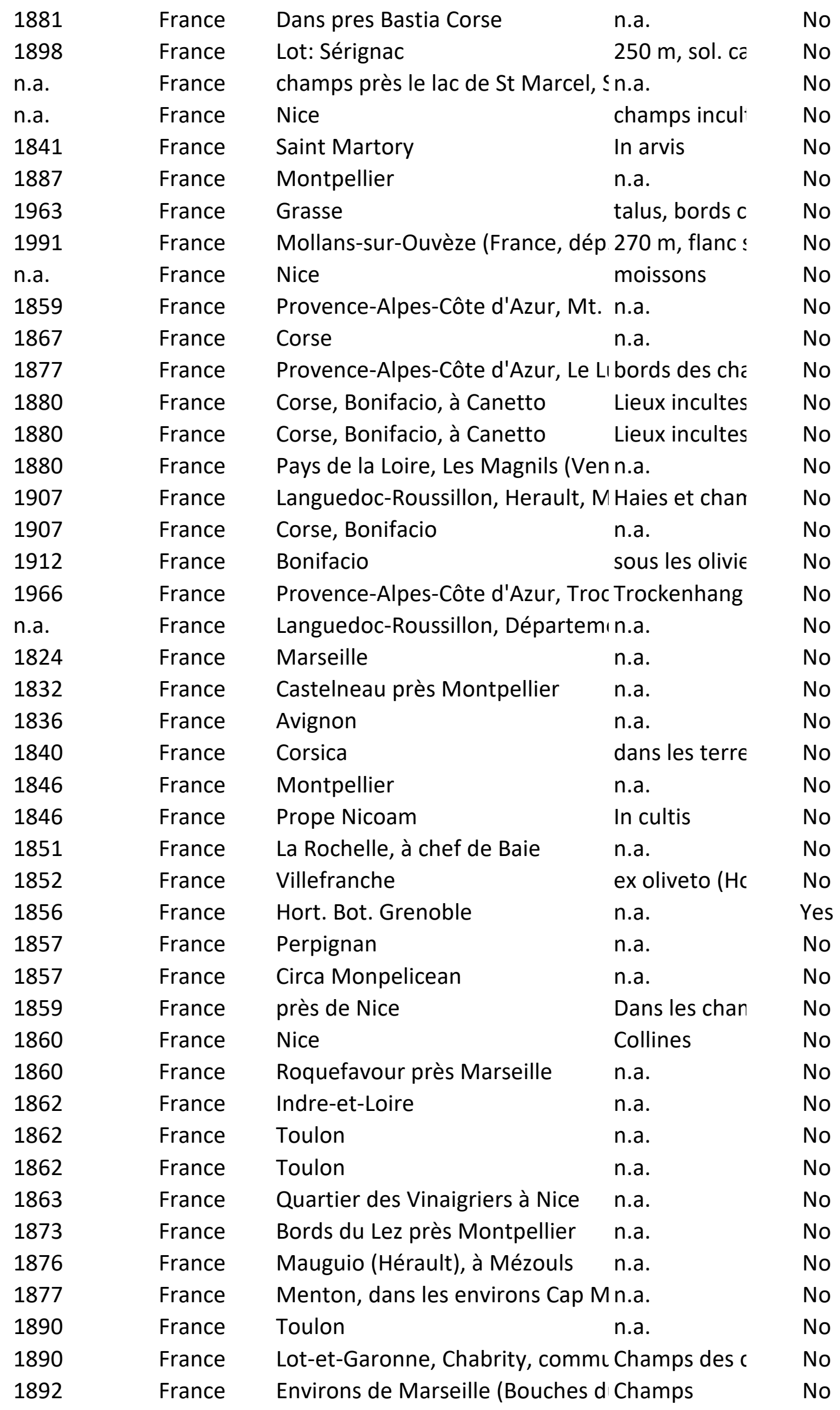




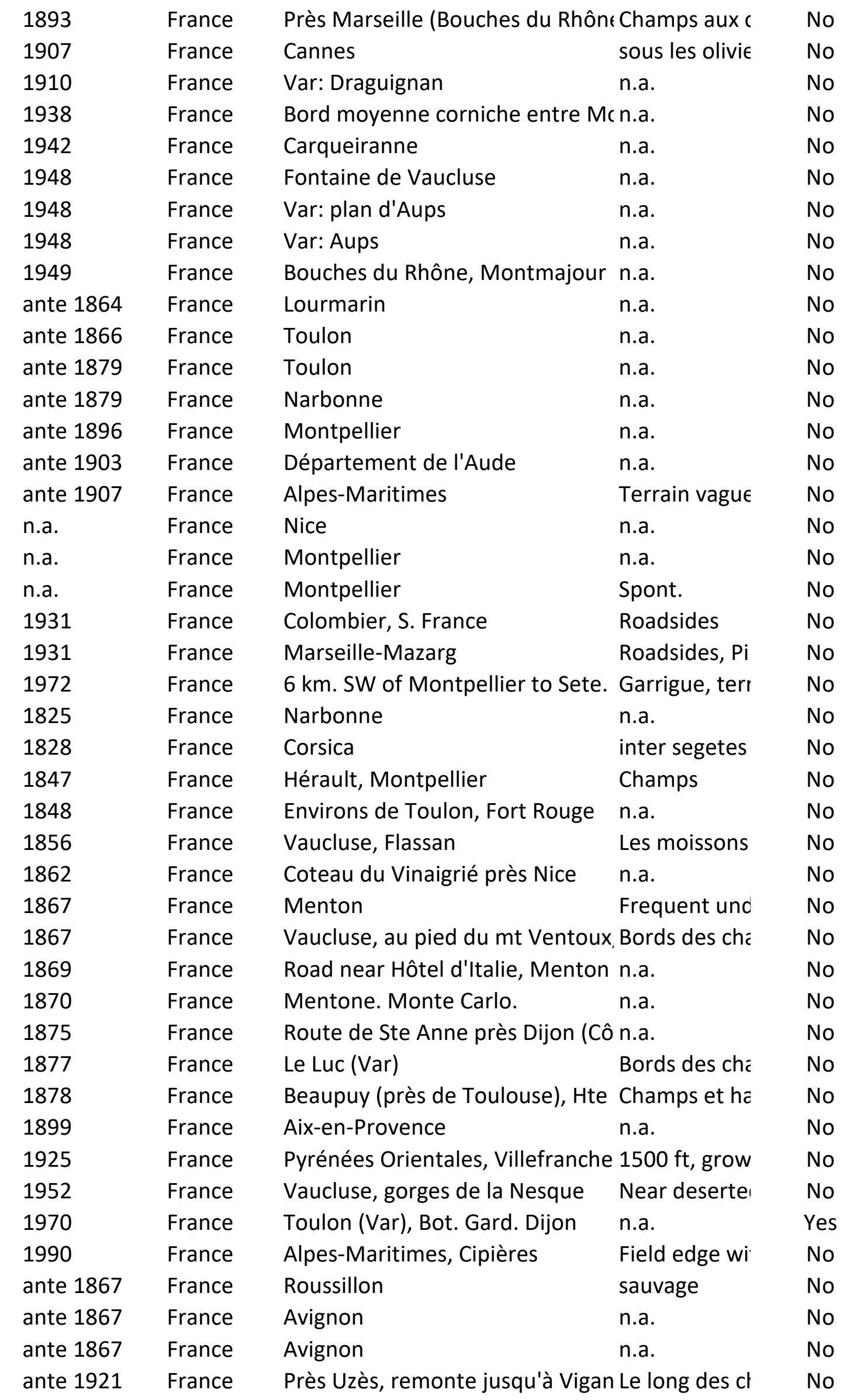




\begin{tabular}{|c|c|c|c|}
\hline n.a. & France & Vaucluse & n.a. \\
\hline n.a. & France & Mentone & n.a. \\
\hline n.a. & France & \multicolumn{2}{|c|}{ Cul ex Sem. Hispanica in Hort. Tin n.a. } \\
\hline 1843 & France & Lyon. Blés près de St. Alban & n.a. \\
\hline 1849 & France & \multicolumn{2}{|c|}{ Champs et vignes près Auch (Ge n.a. } \\
\hline 1891 & France & Bouches-du-Rhône: Aix & bords gazonr \\
\hline 1891 & France & Béziers & n.a. \\
\hline 1930 & France & \multicolumn{2}{|c|}{ Bouches-du-Rhône: La Valentine n.a. } \\
\hline 1961 & France & Les Arisquiers (Hérault) & n.a. \\
\hline 1963 & France & Grasse (AM) & Fossés, lieux \\
\hline 1965 & France & Grasse (AM) & Champs, talu \\
\hline 1965 & France & \multicolumn{2}{|c|}{ Region Provence-Alpes-Cote d'Aichamps incul' } \\
\hline 1965 & France & \multicolumn{2}{|c|}{ Alpes-Maritimes, Villeneuve Loub€ Alt.: 39 m. } \\
\hline 1967 & France & Alpes-Maritimes, Gourdon & Alt.: $800 \mathrm{~m}$ \\
\hline 1967 & France & Alpes Maritimes, Saint Vallier-de & - Alt.: 800 m. \\
\hline 1972 & France & Pova do Conde. Castanheda & n.a. \\
\hline 1991 & France & \multicolumn{2}{|c|}{ Drôme: Mollans-sur-Ouvèze, anci flanc sud du $t$} \\
\hline 1839 & France & \multicolumn{2}{|c|}{ Languedoc-Roussillon, Montpelliє n.a. } \\
\hline 1859 & France & \multicolumn{2}{|c|}{ Languedoc-Roussillon, Montpelliєn.a. } \\
\hline 1862 & France & Coteau du Vinaigrié, près Nice & n.a. \\
\hline n.a. & France & \multicolumn{2}{|c|}{ Provence-Alpes-Cote d'Azur, Mar n.a. } \\
\hline n.a. & France & \multicolumn{2}{|c|}{ Provence-Alpes-Cote d'Azur, Mar n.a. } \\
\hline n.a. & France & \multicolumn{2}{|c|}{ Provence-Alpes-Cote d'Azur, Mar n.a. } \\
\hline n.a. & France & \multicolumn{2}{|c|}{ Provence-Alpes-Cote d'Azur, Proun.a. } \\
\hline 1803 & France & La Rochelle & n.a. \\
\hline 1820 & France & Perpignan, [illegible] & n.a. \\
\hline 1828 & France & Maguelone & n.a. \\
\hline 1834 & France & Monte Grosso près de Nice & n.a. \\
\hline 1835 & France & Fabrègues & champs \\
\hline 1837 & France & \multicolumn{2}{|c|}{ de la gaillarde à la mosson (? Mor bord de chen } \\
\hline 1841 & France & [illegible] & n.a. \\
\hline 1842 & France & Auch (Gers) & n.a. \\
\hline 1846 & France & Toulon, Fort Rouge & n.a. \\
\hline 1847 & France & Montpellier, Hérault & champs \\
\hline 1847 & France & Montpellier & champs \\
\hline 1847 & France & Montpellier & n.a. \\
\hline 1847 & France & Montpellier & champs \\
\hline 1849 & France & environs d'Angoulême & dans les vignt \\
\hline 1849 & France & Puycasquier (Gers) & n.a. \\
\hline 1849 & France & près d'Auch & champs et vi६ \\
\hline 1849 & France & près d'Auch (Gers) & champs et vi६ \\
\hline 1849 & France & Richemont (Charente) & échappée de \\
\hline 1850 & France & Jardin botanique de Lyon & n.a. \\
\hline 1851 & France & \multicolumn{2}{|c|}{ Point de Chef de Baie (La Rochellın.a. } \\
\hline
\end{tabular}




\begin{tabular}{|c|c|c|c|}
\hline 1852 & France & Bourges & décombres aı \\
\hline 1854 & France & Montpellier & n.a. \\
\hline 1858 & France & Montpellier & n.a. \\
\hline 1860 & France & Le Luc (Var) & bords des chí \\
\hline 1860 & France & Le Luc (Var) & bords de chaı \\
\hline 1862 & France & coteau du Vinaigrié près Nice & n.a. \\
\hline 1862 & France & coteau du Vinaigrié près Nice & n.a. \\
\hline 1863 & France & Pézenas & champs, vignı \\
\hline 1864 & France & Antibes & n.a. \\
\hline 1864 & France & Auch & n.a. \\
\hline 1866 & France & Le Luc (Var) & liens incultes \\
\hline 1867 & France & Saint Félix de Lodez & n.a. \\
\hline 1870 & France & Lodève au chemin vieux de Lauro & n.a. \\
\hline 1871 & France & Saint Zacharie (Var) & moissons \\
\hline 1871 & France & Saint Martin de Londres (Hérault) & bords des che \\
\hline 1876 & France & Toulon & champs \\
\hline 1876 & France & Montagne de Cette (= Sète), Héra & n.a. \\
\hline 1876 & France & Montpellier à Montplaisir & haies et char \\
\hline 1876 & France & Montpellier & bord des chel \\
\hline 1877 & France & Flassan & moissons \\
\hline 1877 & France & Le Luc (Var) & bords des chí \\
\hline 1877 & France & Le Luc (Var) & bords des che \\
\hline 1879 & France & Cette (Sète) & n.a. \\
\hline 1880 & France & Bonifacio, à Canetto & lieux incultes \\
\hline 1882 & France & Menton, sur terre italienne bois $C$ & n.a. \\
\hline 1884 & France & Murviel-lez-Montpellier & bords et talus \\
\hline 1884 & France (?) & Alpes (?) & champs mois: \\
\hline 1886 & France & Montbazin (Hérault) & talus du chen \\
\hline 1886 & France & Mausson & n.a. \\
\hline 1886 & France & Béziers, grand fossé du pré-au-nè & n.a. \\
\hline 1887 & France & haies à Balaruc & n.a. \\
\hline 1887 & France & bord chemin Montpellier & n.a. \\
\hline 1887 & France & La Gaillarde & n.a. \\
\hline 1887 & France & Valmaillargues près Montpellier & n.a. \\
\hline 1889 & France & Aniane & bords des chí \\
\hline 1889 & France & Grasse & n.a. \\
\hline 1891 & France & Bouches du Rhône: Aix & bords gazonn \\
\hline 1891 & France & Aix (Bouches-du -Rhône) & bords gazonn \\
\hline 1892 & France & Angoulême & n.a. \\
\hline 1893 & France & Cette (Sète) & coteaux mari \\
\hline 1893 & France & Saint Guilhem le Désert (Hérault) & n.a. \\
\hline 1894 & France & près Angoulême & blés \\
\hline 1894 & France & Seillans (Var) & champs de bl \\
\hline 1896 & France & Agde (Hérault) & n.a. \\
\hline
\end{tabular}




\begin{tabular}{|c|c|c|c|c|}
\hline 1896 & France & \multicolumn{2}{|c|}{ Montpellier, champs de l'école d' n.a. } & No \\
\hline 1898 & France & Nyons (Drôme) & n.a. & No \\
\hline 1898 & France & Nyons (Drôme) & moissons & No \\
\hline 1898 & France & \multicolumn{2}{|c|}{ jardin botanique, graines Monacc n.a. } & No \\
\hline 1898 & France & Millau (Aveyron) & jardin de Natl & Yes \\
\hline 1899 & France & La Nouvelle (Aude) & n.a. & No \\
\hline 1899 & France & La Nouvelle (Aude) & friche & No \\
\hline 1899 & France & Fronsac (Gironde) & champs de bl & No \\
\hline 1899 & France & La Nouvelle (Aude) & friche & No \\
\hline 1901 & France & Corse, Bonifacio & sous les olivi $\epsilon$ & No \\
\hline 1901 & France & La Nouvelle (Aude) & n.a. & No \\
\hline 1901 & France & Corse, Bonifacio & sous les olivi $\epsilon$ & No \\
\hline 1902 & France & environs d'Angoulême & moissons, cot & No \\
\hline 1903 & France & \multicolumn{2}{|c|}{ Corse, Cavallo morto près Bonifac sous les olivi $\epsilon$} & No \\
\hline 1907 & France & Millau (Aveyron) & ravin (?) & No \\
\hline 1907 & France & Léognan (Gironde) & cultivé dans lı & Yes \\
\hline 1914 & France & Darnieux & lieux en frichı & No \\
\hline 1914 & France & Haies à la Coste & n.a. & No \\
\hline 1918 & France & Fréjus & plaine & No \\
\hline 1918 & France & Maraussan \& Ardouane & bord de senti & No \\
\hline 1926 & France & Béziers, en dessous du canal & pentes herbe & No \\
\hline 1930 & France & \multicolumn{2}{|c|}{ La Valentine (Bouches du Rhone) friche à Mont } & No \\
\hline 1936 & France & \multicolumn{2}{|c|}{ Savonnières (Indre-et-Loire), rout pieds des mu } & No \\
\hline 1956 & France & Les Baux du Ventoux & $400 \mathrm{~m}$ & No \\
\hline 1959 & France & \multicolumn{2}{|c|}{ Montpellier, environs hopital sair n.a. } & No \\
\hline 1959 & France & \multicolumn{2}{|c|}{ Montpellier, environs hopital sair n.a. } & No \\
\hline 1959 & France & \multicolumn{2}{|c|}{ Montpellier, hauteurs sur la rive ( $\mathrm{n} . \mathrm{a}}$. & No \\
\hline 1963 & France & Grasse (Alpes-Maritimes) & lieux incultes, & No \\
\hline 1965 & France & Lavalette (près Montpellier) & friches & No \\
\hline 1966 & France & \multicolumn{2}{|c|}{ Murviel-Les-Montpellier (Hérault bord de chan } & No \\
\hline 1966 & France & Vaucluse: Les Baux de Vaucluse & n.a. & No \\
\hline $1893 \& 1898$ & France & \multicolumn{2}{|c|}{ Bordeaux; remblais touchant le p n.a. } & No \\
\hline n.a. & France & [illegible] & n.a. & No \\
\hline n.a. & France & \multicolumn{2}{|c|}{ Près Perpignan, au-delà de la por champs cultiı } & No \\
\hline n.a. & France & Tuchan & n.a. & No \\
\hline n.a. & France & \multicolumn{2}{|c|}{ Béziers, en dessous du canal du n pentes herbe } & No \\
\hline n.a. & France & L'Ardide, près Béziers & talus herbeaı & No \\
\hline n.a. & France & Ardouane & $270 \mathrm{~m}$ & No \\
\hline n.a. & France & Aix en provence & n.a. & No \\
\hline n.a. & France & Sommières (Gard) & champs cultiı & No \\
\hline n.a. & France & Saint-Rémy, Haute Saône & n.a. & No \\
\hline 1784 & France & Jardin de Sally & n.a. & No \\
\hline 1817 & France & Toulon, au bas du Fort rouge & n.a. & No \\
\hline 1821 & France & Toulon, gorges d'Ollioules & n.a. & No \\
\hline
\end{tabular}




\begin{tabular}{|c|c|c|c|}
\hline 1821 & France & Nismes & n.a. \\
\hline 1822 & France & Montpellier & n.a. \\
\hline 1826 & France & cult. Paris & n.a. \\
\hline 1827 & France & Provence & n.a. \\
\hline 1828 & France & Bar sur Aube & L'échantillon \\
\hline 1828 & France & \multicolumn{2}{|c|}{ Courbevoie. Environs de Paris. Han.a. } \\
\hline 1829 & France & Demadou (?) & Plaines et cot \\
\hline 1834 & France & Montpellier & n.a. \\
\hline 1834 & France & Lourmarin & n.a. \\
\hline 1834 & France & Lourmarin & n.a. \\
\hline 1835 & France & Montpellier & n.a. \\
\hline 1836 & France & Grasse & n.a. \\
\hline 1838 & France & Grasse & n.a. \\
\hline 1838 & France & \multicolumn{2}{|c|}{ Besançon (Doubs): jardin du Polyı n.a. } \\
\hline 1842 & France & environs d'Angoulème & dans les chan \\
\hline 1842 & France & Auch (Gers) & n.a. \\
\hline 1843 & France & [illegible] & n.a. \\
\hline 1844 & France & Perpignan, à Castel-Roussillon & n.a. \\
\hline 1845 & France & près Montpellier & Sur le bord $d t$ \\
\hline 1845 & France & \multicolumn{2}{|c|}{ In arvis Puy-conquier (?) (Gers) (? n.a. } \\
\hline 1845 & France & \multicolumn{2}{|c|}{ Montpellier, sur le bord du chemin.a. } \\
\hline 1845 & France & Charente: vignes de Brudy & n.a. \\
\hline 1845 & France & Angoulême, Charente & champs cultil \\
\hline 1845 & France & \multicolumn{2}{|c|}{ Montpellier, sur le bord du chemin.a. } \\
\hline 1845 & France & Toulon & n.a. \\
\hline 1845 & France & Montpellier & sur le bord di \\
\hline 1845 & France & Montpellier & n.a. \\
\hline 1845 & France & Auch & n.a. \\
\hline 1846 & France & \multicolumn{2}{|c|}{ Du fort Rouge, environ de Toulon n.a. } \\
\hline 1846 & France & Environs de Toulon, Fort Rouge & n.a. \\
\hline 1846 & France & Bonifacio (Corse) & n.a. \\
\hline 1846 & France & Edon (Charente) & observée éga \\
\hline 1846 & France & Narbonne & n.a. \\
\hline 1846 & France & Nîmes & Bord des chaı \\
\hline 1847 & France & Environs d'Angoulême & dans les chan \\
\hline 1847 & France & St André près Montpellier & n.a. \\
\hline 1847 & France & Montpellier & Bord des chaı \\
\hline 1847 & France & Montpellier, Hérault & Champs \\
\hline 1847 & France & Montpellier, Hérault & Champs \\
\hline 1847 & France & Montpellier (Hérault) & champs \\
\hline 1848 & France & Fort rouge & France mérid \\
\hline 1848 & France & Env. de Toulon, Fort rouge & n.a. \\
\hline 1848 & France & Fort rouge & n.a. \\
\hline 1848 & France & Env. de Toulon, Fort rouge & n.a. \\
\hline
\end{tabular}




\begin{tabular}{|c|c|c|c|}
\hline 1849 & France & Auch (Gers) & champs près \\
\hline 1849 & France & Auch (Gers) & Champs et vi६ \\
\hline 1849 & France & Auch (Gers) & Champs et vi६ \\
\hline 1850 & France & Agen & champ cultive \\
\hline 1850 & France & Linon & dans les mois \\
\hline 1850 & France & Env. d'Angoulême & n.a. \\
\hline 1850 & France & Montpellier (Hérault) & n.a. \\
\hline 1850 & France & Montpellier, champs & n.a. \\
\hline 1851 & France & Chatillon & Plateau de $\mathrm{Cr}$ \\
\hline 1851 & France & La Rochelle & n.a. \\
\hline 1852 & France & Dans quelques champs seulemen & Mr. Letourne \\
\hline 1852 & France & Les Magnils (Vendée) & près Luçon, $d$ \\
\hline 1853 & France & Lot-et-Garonne. Corne, près de C & n.a. \\
\hline 1854 & France & Saintes (Charente maritime) & Spontanée dé \\
\hline 1854 & France & Auch & moissons (spr \\
\hline 1854 & France & Auch & n.a. \\
\hline 1856 & France & Bastia, lieux incultes & n.a. \\
\hline 1856 & France & Environs de Luçon (Vendée) & n.a. \\
\hline 1856 & France & n.a. & hortus andeg \\
\hline 1858 & France & Pas de Calais. Etaples. & Cultivée \\
\hline 1859 & France & Toulon, les Sablettes & n.a. \\
\hline 1859 & France & Villeneuve les maguelonne (Héra। & in.a. \\
\hline 1860 & France & Montredon & n.a. \\
\hline 1860 & France & Montredon & n.a. \\
\hline 1860 & France & Luc (Var) & Bords des ché \\
\hline 1860 & France & Luc (Var) & Bords des chí \\
\hline 1860 & France & Luc (Var) & Bords des chí \\
\hline 1860 & France & Villanière, collines sèches & Plantes du ve \\
\hline 1860 & France & Villanière, collines sèches & Plantes du ve \\
\hline 1860 & France & Antibes (Alpes-Maritimes) & n.a. \\
\hline 1860 & France & Montredon près Marseille & n.a. \\
\hline 1860 & France & Le Luc (Var) & Bords des chí \\
\hline 1860 & France & Le Luc (Var) & Bords des chí \\
\hline 1860 & France & Le Luc (Var) & Bords des chí \\
\hline 1860 & France & Montredon: collines & n.a. \\
\hline 1860 & France & St Pons (Bouches du Rhône) & Vallon de St F \\
\hline 1861 & France & Toulon & n.a. \\
\hline 1861 & France & Nice (Alpes Maritimes) & champs incult \\
\hline 1861 & France & Nice & Champs incul \\
\hline 1861 & France & Nice & Champs incul \\
\hline 1861 & France & Nice & Champs incul \\
\hline 1861 & France & Nice & Champs incul \\
\hline 1862 & France & Nice (Alpes Maritimes) & Coteau du Vir \\
\hline 1862 & France & Coteau du Vinaigrié près Nice & n.a. \\
\hline
\end{tabular}




\begin{tabular}{|c|c|c|c|}
\hline 1862 & France & Coteau du Vinaigrié près Nice & n.a. \\
\hline 1862 & France & Coteau du Vinaigrié près Nice & n.a. \\
\hline 1862 & France & Coteau du Vinaigrié près Nice & n.a. \\
\hline 1862 & France & Nice (Alpes Maritimes) & coteau du 'Vi \\
\hline 1862 & France & Béziers & n.a. \\
\hline 1862 & France & Coteau du Vinaigrié près Nice & n.a. \\
\hline 1862 & France & Rivesaltes & n.a. \\
\hline 1862 & France & \multicolumn{2}{|l|}{ Aveyron: gorges d'Estrabols, $\mathrm{C} \underline{\mathrm{m}}$ c n.a. } \\
\hline 1863 & France & Cavaillon, Vaucluse & n.a. \\
\hline 1863 & France & \multicolumn{2}{|l|}{ Montagne de St Jacques sur Cava n.a. } \\
\hline 1863 & France & Grabels & moissons à G \\
\hline 1865 & France & Ile Ste Marguerite & n.a. \\
\hline 1865 & France & Sous les oliviers, près de Menton r & n.a. \\
\hline 1866 & France & Lévignen (Oise) & Moissons \\
\hline 1866 & France & Tayac, les Eyzies (Dordogne) & n.a. \\
\hline 1866 & France & [illegible] & région des oli \\
\hline 1866 & France & \multicolumn{2}{|c|}{ Ile verte près La Ciotat, Bouches c n.a. } \\
\hline 1866 & France & St Remy (Haute Saône) & château, $2 \mathrm{m \epsilon}$ \\
\hline 1867 & France & Vaucluse & n.a. \\
\hline 1867 & France & près Nice & Iter italicum \\
\hline 1868 & France & n.a. & champs, cult. \\
\hline 1869 & France & Corse, Bastia & colline sèche \\
\hline 1869 & France & Nemours & n.a. \\
\hline 1869 & France & Besançon & cult. ornt. \\
\hline 1870 & France & Agen & n.a. \\
\hline 1870 & France & Crest (Drôme) & moissons \\
\hline 1870 & France & Crest & moissons \\
\hline 1871 & France & Chatillon (Seine) & Redoute de C \\
\hline 1871 & France & Orange (Vaucluse) & Mourrarouge \\
\hline 1871 & France & Les Magnils (Vendée) & près Luçon, d \\
\hline 1872 & France & Cheverny (Loir et Cher) & Herbier de pli \\
\hline 1873 & France & Vaucluse & bord de la roı \\
\hline 1873 & France & Jourdan & sans doute éc \\
\hline 1874 & France & Toulon (Var) & lieux incultes \\
\hline 1875 & France & Env. de Cette (Hérault) & n.a. \\
\hline 1875 & France & Gorniès (Hérault) & moissons, prè \\
\hline 1875 & France & Montpellier & n.a. \\
\hline 1875 & France & Les Magnisles, près Luçon & n.a. \\
\hline 1875 & France & \multicolumn{2}{|l|}{ Le Luc (Var), haies et lieux inculte n.a. } \\
\hline 1875 & France & Clermont & subspontanéı \\
\hline 1877 & France & Le Luc (Var) & bords des chí \\
\hline 1877 & France & Le Luc (Var): bord des champs & n.a. \\
\hline 1877 & France & Le Luc (Var) & bords des chí \\
\hline 1877 & France & Le Luc (Var) & bords des ché \\
\hline
\end{tabular}




\begin{tabular}{|c|c|c|c|}
\hline 1877 & France & Flassan & moissons \\
\hline 1877 & France & Beaupuy (près de Toulouse) & n.a. \\
\hline 1878 & France & Montagne de Celle (Hérault) & n.a. \\
\hline 1878 & France & Beaupuy (Haute Garonne) & Champs et ha \\
\hline 1878 & France & Beaupuy (Haute Garonne) & Champs et ha \\
\hline 1878 & France & Beaupuy (Haute Garonne) & Champs et ha \\
\hline 1878 & France & Millau & n.a. \\
\hline 1878 & France & \multicolumn{2}{|c|}{ Monsempron Libos (Lot-et-Garon n.a. } \\
\hline 1880 & France & Corse, Bonifacio, à Canetto & Lieux incultes \\
\hline 1880 & France & Corse, Bonifacio, à Canetto & Lieux incultes \\
\hline 1880 & France & Corse, Bonifacio, à Canetto & Lieux incultes \\
\hline 1880 & France & Bonifacio (Corse) & lieux incultes, \\
\hline 1881 & France & Drap (06) & région littora \\
\hline 1883 & France & Saint-Emiland (S. et Loire) & cultivé de gra \\
\hline 1883 & France & Saint-Emiland (S. et Loire) & cultivé de gra \\
\hline 1883 & France & La Huberdière & Jardins \\
\hline 1885 & France & Montpellier & n.a. \\
\hline 1886 & France & Sérignan (Vaucluse) & Bord des chaı \\
\hline 1886 & France & Fontan & Alpes-Maritin \\
\hline 1886 & France & Fontan & moissons \\
\hline 1886 & France & n.a. & n.a. \\
\hline 1886 & France & Belle Ile en Mer (Morbihan) & provenant de \\
\hline 1886 & France & \multicolumn{2}{|c|}{ Originaire de l'Est de l'Espagne; cı Fleurs 22 juill } \\
\hline 1886 & France & \multicolumn{2}{|c|}{ Originaire de l'Est de l'Espagne; cı Fleurs 22 juill } \\
\hline 1886 & France & \multicolumn{2}{|c|}{ Saône et Loire: cultivé à Saint-Em Fleurs 22 juill } \\
\hline 1887 & France & \multicolumn{2}{|c|}{ Carcassone (Aude), moissons vers n.a. } \\
\hline 1888 & France & Annecy & carrières \\
\hline 1888 & France & Clamart & dans les jardi \\
\hline 1889 & France & Montpellier (Hérault) & route de Gan \\
\hline 1891 & France & Aix (Bouches du Rhône) & bords gazonn \\
\hline 1891 & France & Bouches-du-Rhône: Aix & bords gazonn \\
\hline 1891 & France & Bouches-du-Rhône: Aix & bords gazonn \\
\hline 1891 & France & Bouches-du-Rhône: Aix & bords gazonn \\
\hline 1891 & France & Pointe du Chef de Baie & n.a. \\
\hline 1892 & France & Montboron (Alpes Maritimes) & région littora \\
\hline 1893 & France & St Remy & cult. ornt. \\
\hline 1894 & France & Fontaine de Vaucluse & n.a. \\
\hline 1895 & France & Nice (Alpes Maritimes) & région littora \\
\hline 1897 & France & Louis (Pyrénées orientales) & champs, vignı \\
\hline 1897 & France & Antibes & ça et là dans I \\
\hline 1897 & France & Mayenne & sur le chemin \\
\hline 1898 & France & Salavre (Ain) & terre sablonn \\
\hline 1898 & France & \multicolumn{2}{|c|}{ Corse: Aleria, domaine de Casabi氵 n.a. } \\
\hline 1899 & France & Fronsac (Gironde) & les champs dt \\
\hline
\end{tabular}




\begin{tabular}{|c|c|c|c|}
\hline 1899 & France & La nouvelle (Aude) & var. minor Bo \\
\hline 1899 & France & \multicolumn{2}{|c|}{ Arsyron: Peyre, route de Compré! Var. minor $B c$} \\
\hline 1899 & France & Chartres (Eure et Loir) & chemin, longt \\
\hline 1900 & France & Fronsac (Gironde) & champs calca \\
\hline 1900 & France & n.a. & n.a. \\
\hline 1900 & France & Millau (Aveyron) & côte de St Est \\
\hline 1901 & France & Corse: Bonifacio & sous les olivi $\epsilon$ \\
\hline 1901 & France & Corse: Bonifacio & sous les olivi $\epsilon$ \\
\hline 1901 & France & Bonifacio (Corse) & sous les olivi \\
\hline 1901 & France & La Nouvelle (Aude) & var. minor Bo \\
\hline 1902 & France & Angoulême & moissons calc \\
\hline 1902 & France & Dord. Champs de Granbges & n.a. \\
\hline 1902 & France & Aude: La Nouvelle & n.a. \\
\hline 1902 & France & Grasse (Alpes Maritimes) & de Mouans Si \\
\hline 1902 & France & St Germain (Seine et Oise) & n.a. \\
\hline 1902 & France & St Germain (Seine et Oise) & n.a. \\
\hline 1903 & France & \multicolumn{2}{|c|}{ Le Val d'CEuf, Cne de Mougins (Al n.a. } \\
\hline 1903 & France & Cette & n.a. \\
\hline 1905 & France & Environs de Marseille & (Sté bat. de F \\
\hline 1905 & France & Tarbes (Hautes Pyrénées) & route de Pau \\
\hline 1905 & France & Montceau-les-Mines & Plante méridi \\
\hline 1905 & France & Montceau-les-Mines & Plante méridi \\
\hline 1906 & France & Sète (Hérault) & Montagne de \\
\hline 1906 & France & Sannois (S. et O.) & n.a. \\
\hline 1907 & France & Montpellier (Hérault) & haies \\
\hline 1909 & France & Garches & champ incultı \\
\hline 1909 & France & Saint-Tropez, Sainte-Anne & n.a. \\
\hline 1910 & France & Duran près Auch (Gers) & n.a. \\
\hline 1910 & France & \multicolumn{2}{|c|}{ Entre Béziers et Agde, garrigues on.a. } \\
\hline 1911 & France & \multicolumn{2}{|c|}{ Saint-Tropez. Var. Vallon St Antoi n.a. } \\
\hline 1911 & France & St Tropez & n.a. \\
\hline 1911 & France & Saint-Joseph, Saint-Tropez. Var & n.a. \\
\hline 1911 & France & Saint-Gilles / Gard & n.a. \\
\hline 1912 & France & Groslay & bord de la vo \\
\hline 1912 & France & Ezanville & champ en bol \\
\hline 1914 & France & Béziers (Hérault) & tertres herbe \\
\hline 1914 & France & Béziers (Hérault) & tertres herbe \\
\hline 1914 & France & près de Chaix, Vendée & sur la route $d$ \\
\hline 1917 & France & Alpes-Maritimes: Vence & n.a. \\
\hline 1917 & France & La Clarté, près Perros Guirrec & bord des chel \\
\hline 1919 & France & Brignoles (Var) & coteaux vers \\
\hline 1920 & France & Versailles & n.a. \\
\hline 1922 & France & La Mothe St Héray (Deux Sèvres) & dans un jardiı \\
\hline 1923 & France & Nice (Alpes Maritimes) & champs \\
\hline
\end{tabular}




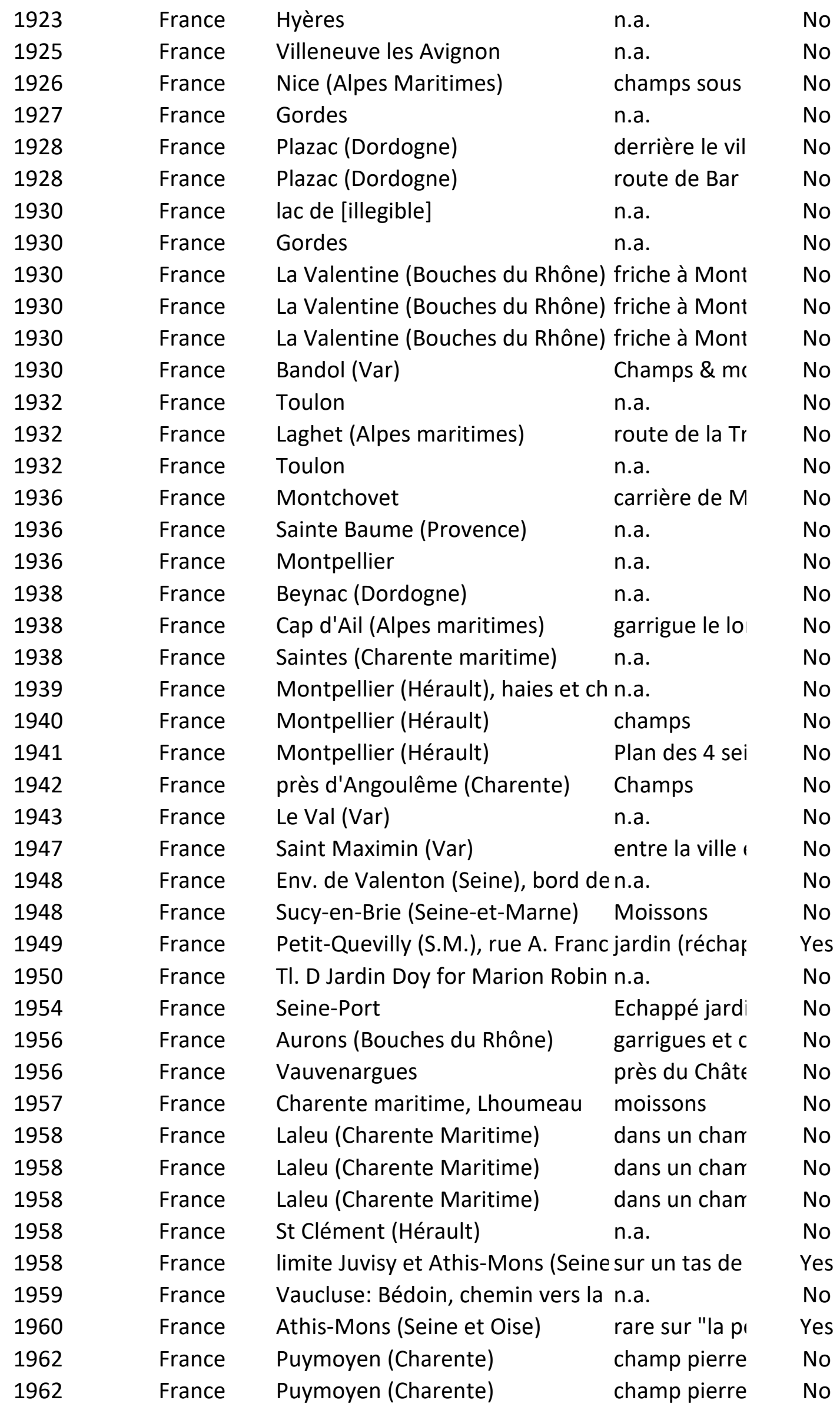




\begin{tabular}{|c|c|c|c|}
\hline 1963 & France & Grasse (Alpes Maritimes) & lieux incultes, \\
\hline 1963 & France & Grasse (Alpes Maritimes) & lieux incultes, \\
\hline 1969 & France & \multicolumn{2}{|c|}{ Athis-Mons, à peu de distance du involucre pré } \\
\hline 1969 & France & \multicolumn{2}{|c|}{ Athis-Mons (Essone), terrain encc un seul indivi } \\
\hline 1971 & France & \multicolumn{2}{|c|}{ Montigny-sur-Loing (Seine-et-Ma Marge de la $r$} \\
\hline 1973 & France & \multicolumn{2}{|c|}{ Montelimar (Ardèche-Drôme). La n.a. } \\
\hline 1973 & France & \multicolumn{2}{|c|}{ Montelimar (Ardèche-Drôme). La n.a. } \\
\hline 1975 & France & \multicolumn{2}{|c|}{ préalpes de Grasse. Le Cheiron-gen.a. } \\
\hline 1990 & France & Alpes-Maritimes, Cipières. & Field edge wi \\
\hline 1991 & France & Mollans-sur-Ouvèze (Drôme) & ancienne voit \\
\hline 2001 & France & Amboise (F37) & race cultivée \\
\hline 2009 & France & Orconte (51300) & spontanée d $\epsilon$ \\
\hline 2015 & France & \multicolumn{2}{|c|}{ Cultivé à: UMR 0320/8120, Génét Morphe [T], c } \\
\hline 2015 & France & \multicolumn{2}{|c|}{ Cultivé à: UMR 0320/8120, Génét Morphe [T], c } \\
\hline 2015 & France & \multicolumn{2}{|c|}{ Cultivé à: UMR 0320/8120, Génét Morphe [P], c } \\
\hline 2015 & France & \multicolumn{2}{|c|}{ Cultivé à: UMR 0320/8120, Génét Morphe [P], C } \\
\hline 2015 & France & \multicolumn{2}{|c|}{ Cultivé à: UMR 0320/8120, Génét Morphe [P], c } \\
\hline 2015 & France & \multicolumn{2}{|c|}{ Cultivé à: UMR 0320/8120, Génét Morphe [T], c } \\
\hline 2015 & France & \multicolumn{2}{|c|}{ Cultivé à: UMR 0320/8120, Génét Morphe [T], c } \\
\hline 2015 & France & \multicolumn{2}{|c|}{ Cultivé à: UMR 0320/8120, Génét Morphe [T], c } \\
\hline 2015 & France & \multicolumn{2}{|c|}{ Cultivé à: UMR 0320/8120, Génét Morphe [T], c } \\
\hline ante 1847 & France & n.a. & fl. albo et fl. c \\
\hline ante 1868 & France & Notre Dame de Pau (py or) & n.a. \\
\hline ante 1949 & France & Nice & n.a. \\
\hline n.a. & France & Montpellier & n.a. \\
\hline n.a. & France & Morée & Morée Cranic \\
\hline n.a. & France & La Seyne (Var) & fl. Mai-juillet \\
\hline n.a. & France & France méridionale. & n.a. \\
\hline n.a. & France & \multicolumn{2}{|c|}{ J'ai trouvé cette plante dans les c n.a. } \\
\hline n.a. & France & Montarin & n.a. \\
\hline n.a. & France & Bonifacio & Var. pallifilor: \\
\hline n.a. & France & Env. de Montpellier & n.a. \\
\hline n.a. & France & in Gall Narbonnes (?) & n.a. \\
\hline n.a. & France & ruines du château d'Hyères & n.a. \\
\hline n.a. & France & Nice (Alpes Maritimes) & moissons \\
\hline n.a. & France & Montpellier (Hérault) & n.a. \\
\hline n.a. & France & n.a. & n.a. \\
\hline n.a. & France & Saintes Chte (?) & n.a. \\
\hline n.a. & France & Perpignan & n.a. \\
\hline n.a. & France & Bonifacio & n.a. \\
\hline n.a. & France & Avignon (Vaucluse) & n.a. \\
\hline n.a. & France & Bord de l'Allier & n.a. \\
\hline n.a. & France & Luçon & Lou Bourdeaı \\
\hline n.a. & France & Environs de Rennes & n.a. \\
\hline
\end{tabular}




\begin{tabular}{|c|c|c|c|}
\hline n.a. & France & n.a. & Herb. Univ. Ci \\
\hline n.a. & France & France méridionale & Cultivée dans \\
\hline 1861 & France & \multicolumn{2}{|c|}{ Aveyron: Canton de Villeneuve, e n.a. } \\
\hline 1862 & France & \multicolumn{2}{|c|}{ Aveyron: Combes d'Estrabols, Ce Au fond du ré } \\
\hline 1880 & France & Corse. Bonifacio a Canetto & n.a. \\
\hline 1883 & France & Gallica. Luçon & n.a. \\
\hline 1883 & France & \multicolumn{2}{|c|}{ Provence-Alpes-Cote d'Azur-Men n.a. } \\
\hline 1891 & France & Bouches-du-Rhone: Aix & bords gazonn \\
\hline 1903 & France & \multicolumn{2}{|c|}{ Coutronne (massif de la S: tb=Baı n.a. } \\
\hline 1906 & France & Menton. Alp. Mar & n.a. \\
\hline 1911 & France & Bretagne, Frankrike, Benodet & n.a. \\
\hline 1911 & France & Bretagne, Frankrike, Benodet & n.a. \\
\hline n.a. & France & \multicolumn{2}{|c|}{ Midi-Pyrénées: Champs et vignes n.a. } \\
\hline n.a. & France & Montpellier & n.a. \\
\hline 1872 & France & Nizza: in arvis & n.a. \\
\hline 1873 & France & Nice, sur le Mont Alban & n.a. \\
\hline 1882 & France & \multicolumn{2}{|c|}{ champs, Marseille, bouches du rhn.a. } \\
\hline 1895 & France & Littoral, Nice Montgros & n.a. \\
\hline 1903 & France & \multicolumn{2}{|c|}{ Corse: sous les oliviers de Cavallo n.a. } \\
\hline 1823 & France & \multicolumn{2}{|c|}{ Provence-Alpes-Côte d'Azur: Bouın.a. } \\
\hline 1846 & France & \multicolumn{2}{|c|}{ Languedoc-Roussillon: Hérault, N n.a. } \\
\hline 1855 & France & \multicolumn{2}{|c|}{ Provence-Alpes-Côte d'Azur: Var, Lieux pierreu: } \\
\hline 1869 & France & \multicolumn{2}{|c|}{ Languedoc-Roussillon: Hérault, Bın.a. } \\
\hline 1872 & France & \multicolumn{2}{|c|}{ Provence-Alpes-Côte d'Azur: Bou Lieux incultes } \\
\hline 1880 & France & \multicolumn{2}{|c|}{ Pays de la Loire: Vendée, Les Mą̨ Inter segetes. } \\
\hline 1883 & France & \multicolumn{2}{|c|}{ Provence-Alpes-Côte d'Azur: Alpєn.a. } \\
\hline 1886 & France & \multicolumn{2}{|c|}{ Provence-Alpes-Côte d'Azur: Alpє Moissons } \\
\hline 1893 & France & \multicolumn{2}{|c|}{ Provence-Alpes-Côte d'Azur: Alpєn.a. } \\
\hline n.a. & France & \multicolumn{2}{|c|}{ Languedoc-Roussillon: Hérault, N n.a. } \\
\hline 1849 & France & Champs près auch & n.a. \\
\hline 1849 & France & \multicolumn{2}{|c|}{ Champs et vignes prés Auch (Ger.n.a. } \\
\hline 1849 & France & \multicolumn{2}{|c|}{ Champs et vignes prés Auch (Ger.n.a. } \\
\hline 1874 & France & \multicolumn{2}{|c|}{ Champs à Gleizé, près Villefranch n.a. } \\
\hline 1882 & France & \multicolumn{2}{|c|}{ Les Magnils prope Luçon (Vendé $€$ in agris triticc } \\
\hline 1895 & France & Le Luc (Var) & haies, lieux in \\
\hline 1911 & France & Bouches du Rhone: Luynes & champs et lie \\
\hline 1944 & France & Hérault: Montpellier & n.a. \\
\hline 1947 & France & Saint Maximin & n.a. \\
\hline 1965 & France & Fundort: Dep. Var: 1-2 km Le Luc & n.a. \\
\hline 1977 & France & Perpignan, campus Univ & n.a. \\
\hline
\end{tabular}




\section{Cultivated? (¿Flower preseı Flower morpl Presence of c GPS}

\begin{tabular}{|c|c|c|c|c|}
\hline No & Yes & $P$ & No & $43.646111,0.586667$ \\
\hline No & Yes & $P$ & Yes & $43.646111,0.586667$ \\
\hline No & Yes & $P$ & Yes & $43.710287,7.305119$ \\
\hline No & Yes & $P$ & Yes & $43.710287,7.305119$ \\
\hline No & Yes & $P$ & Yes & $/ /$ \\
\hline Yes & Yes & $\mathrm{T}$ & No & $/ /$ \\
\hline No & Yes & $P$ & No & $43.610556,3.876667$ \\
\hline Yes & Yes & $\mathrm{T}$ & No & // \\
\hline No & Yes & $\mathrm{T}$ & Yes & $48.573405,7.752111$ \\
\hline No & No & n.a. & Yes & $47.795111,1.01964$ \\
\hline No & No & n.a. & Yes & $47.795111,1.01964$ \\
\hline No & Yes & $P$ & No & $44.239147,5.126847$ \\
\hline No & Yes & $\mathrm{T}$ & Yes & $44.732496,-0.462423$ \\
\hline No & No & n.a. & Yes & $45.043983,-0.557815$ \\
\hline No & No & n.a. & Yes & $43.605792,1.499266$ \\
\hline No & Yes & $\mathrm{P}$ & Yes & $43.021111,3.043889$ \\
\hline No & Yes & $P$ & Yes & $41.387174,9.159269$ \\
\hline No & Yes & $P$ & Yes & $41.387174,9.159269$ \\
\hline No & Yes & $P$ & Yes & $47.384882,0.554293$ \\
\hline No & Yes & n.a & No & $43.606476,5.756593$ \\
\hline No & Yes & $P$ & Yes & $43.296389,5.483333$ \\
\hline No & Yes & $P$ & No & $43.660153,6.926492$ \\
\hline No & Yes & $P$ & No & $45.764043,4.835659$ \\
\hline No & Yes & $P$ & No & $44.239147,5.126847$ \\
\hline No & Yes & $P$ & Yes & $43.114379,6.183692$ \\
\hline No & Yes & $P$ & No & $43.142984,5.916093$ \\
\hline No & Yes & $\mathrm{P}$ & Yes & $45.744161,-0.631667$ \\
\hline No & Yes & $P$ & No & $43.603911,1.448975$ \\
\hline No & Yes & $P$ & No & $42.974942,9.429053$ \\
\hline No & Yes & $P$ & No & $43.154306,5.945556$ \\
\hline No & Yes & $P$ & Yes & $43.515225,5.306681$ \\
\hline No & Yes & $P$ & Yes & $43.393722,6.316657$ \\
\hline No & Yes & $\mathrm{P} / \mathrm{T}$ & Yes & $43.621975,5.299489$ \\
\hline No & Yes & $P$ & Yes & $45.926661,-1.008539$ \\
\hline No & Yes & $P$ & Yes & $42.692003,9.450392$ \\
\hline No & Yes & $\mathrm{P} / \mathrm{T}$ & No & $42.692003,9.450392$ \\
\hline No & Yes & $P$ & Yes & $43.100242,6.133344$ \\
\hline No & Yes & $P$ & Yes & $43.891711,3.632081$ \\
\hline No & Yes & $P$ & Yes & $43.393722,6.316657$ \\
\hline No & Yes & $P$ & No & $43.650664,1.55445$ \\
\hline No & Yes & $P$ & No & $41.422981,9.220775$ \\
\hline No & Yes & $P$ & No & $43.125556,6.13$ \\
\hline
\end{tabular}




\begin{tabular}{|c|c|c|c|c|}
\hline No & No & n.a. & Yes & $46.166667,-1.147458$ \\
\hline No & Yes & $\mathrm{T}$ & No & // \\
\hline No & Yes & $P$ & No & $46.162808,-1.205111$ \\
\hline No & Yes & $P$ & Yes & $46.572727,-0.964886$ \\
\hline No & Yes & $P$ & No & $43.499933,3.756367$ \\
\hline No & Yes & $P$ & Yes & $43.710173,7.261953$ \\
\hline No & Yes & $P$ & No & $43.380042,3.211053$ \\
\hline No & Yes & $\mathrm{P}$ & No & $43.766328,7.432408$ \\
\hline No & Yes & $P$ & No & $42.800797,2.498919$ \\
\hline No & Yes & $P$ & No & $43.296389,5.483333$ \\
\hline No & Yes & $P$ & No & $43.296389,5.483333$ \\
\hline No & Yes & $P$ & Yes & $44.239147,5.126847$ \\
\hline No & Yes & $P$ & No & $42.688659,2.894833$ \\
\hline No & Yes & $P$ & No & $43.184277,3.003078$ \\
\hline No & Yes & $?$ & No & $43.646111,0.586667$ \\
\hline No & No & n.a. & Yes & $43.949317,4.805528$ \\
\hline No & Yes & $P$ & No & $43.184277,3.003078$ \\
\hline No & Yes & $P$ & No & $43.184277,3.003078$ \\
\hline No & Yes & $P$ & No & $43.127395,2.898443$ \\
\hline No & Yes & $P$ & No & $42.688659,2.894833$ \\
\hline No & Yes & $P$ & No & $42.688659,2.894833$ \\
\hline No & Yes & $P$ & No & $43.124228,5.928$ \\
\hline No & Yes & $P$ & No & $43.718565,2.072531$ \\
\hline No & Yes & $P$ & No & $43.667285,4.061846$ \\
\hline No & Yes & $P$ & No & $42.692003,9.450392$ \\
\hline No & Yes & $P$ & Yes & $43.393722,6.316657$ \\
\hline No & No & n.a. & Yes & 41.422981, 9.220775 \\
\hline No & Yes & $P$ & No & 41.422981, 9.220775 \\
\hline No & Yes & $P$ & Yes & $44.002722,7.554131$ \\
\hline No & Yes & $P$ & Yes & $44.002722,7.554131$ \\
\hline No & Yes & $\mathrm{P}$ & Yes & $44.002722,7.554131$ \\
\hline No & Yes & $P$ & No & $43.610556,3.876667$ \\
\hline No & Yes & $P$ & No & $43.710173,7.261953$ \\
\hline No & No & n.a. & Yes & $43.763477,5.362582$ \\
\hline No & Yes & $P$ & Yes & $43.706718,7.298076$ \\
\hline No & Yes & $P$ & Yes & $43.710173,7.261953$ \\
\hline No & Yes & $P$ & Yes & $44.658341,0.060664$ \\
\hline No & Yes & $P$ & Yes & $43.646111,0.586667$ \\
\hline No & Yes & $P$ & Yes & 42.039604, 9.012893 \\
\hline No & Yes & $P$ & Yes & $46.454772,-1.167062$ \\
\hline No & Yes & $P$ & No & $43.710173,7.261953$ \\
\hline No & Yes & $\mathrm{T}$ & No & $44.728275,5.024072$ \\
\hline No & Yes & $\mathrm{T}$ & Yes & $44.728275,5.024072$ \\
\hline No & Yes & $P$ & Yes & $43.393722,6.316657$ \\
\hline
\end{tabular}




\begin{tabular}{|c|c|c|c|c|}
\hline No & Yes & $P$ & Yes & $42.692003,9.450392$ \\
\hline No & Yes & $\mathrm{T}$ & Yes & $43.920553,1.022943$ \\
\hline No & No & n.a. & Yes & $45.511935,6.566843$ \\
\hline No & Yes & $\mathrm{P}$ & Yes & $43.710173,7.261953$ \\
\hline No & Yes & $\mathrm{T}$ & Yes & $43.143227,0.930699$ \\
\hline No & Yes & $P$ & Yes & $43.610556,3.876667$ \\
\hline No & Yes & $P$ & No & $43.660153,6.926492$ \\
\hline No & Yes & $P$ & No & $44.239147,5.126847$ \\
\hline No & Yes & $P$ & No & $43.710173,7.261953$ \\
\hline No & Yes & $P$ & Yes & $43.154306,5.945556$ \\
\hline No & Yes & $P$ & No & $42.039604,9.012893$ \\
\hline No & Yes & $P$ & Yes & $43.393722,6.316657$ \\
\hline No & Yes & $P$ & Yes & $41.422981,9.220775$ \\
\hline No & Yes & $P$ & Yes & 41.422981, 9.220775 \\
\hline No & Yes & $\mathrm{P}$ & Yes & $46.572727,-0.964886$ \\
\hline No & No & n.a. & Yes & $43.610556,3.876667$ \\
\hline No & No & n.a. & Yes & $41.387174,9.159269$ \\
\hline No & Yes & $\mathrm{P}$ & No & 41.387174, 9.159269 \\
\hline No & Yes & $P$ & No & $43.710173,7.261953$ \\
\hline No & Yes & $P$ & No & $43.992927,3.606322$ \\
\hline No & Yes & $P$ & Yes & $43.3,5.383333333$ \\
\hline No & Yes & $P$ & No & $43.638755,3.913851$ \\
\hline No & Yes & $P$ & No & $43.949317,4.805528$ \\
\hline No & Yes & $P$ & No & 42.039604, 9.012893 \\
\hline No & Yes & $P$ & No & $43.610556,3.876667$ \\
\hline No & Yes & $P$ & Yes & $43.710173,7.261953$ \\
\hline No & Yes & $\mathrm{T}$ & No & $46.160329,-1.151139$ \\
\hline No & Yes & $P$ & Yes & $42.529047,2.248169$ \\
\hline Yes & Yes & $P$ & Yes & // \\
\hline No & Yes & $P$ & Yes & $42.688659,2.894833$ \\
\hline No & Yes & $P$ & No & $43.610556,3.876667$ \\
\hline No & Yes & $P$ & Yes & $43.710173,7.261953$ \\
\hline No & Yes & $?$ & Yes & $43.710173,7.261953$ \\
\hline No & Yes & $P$ & Yes & $43.545232,5.286626$ \\
\hline No & No & n.a. & Yes & $47.245995,0.632094$ \\
\hline No & Yes & $\mathrm{P}$ & Yes & $43.124228,5.928$ \\
\hline No & Yes & $P$ & No & $43.124228,5.928$ \\
\hline No & Yes & $P$ & No & $43.710287,7.305119$ \\
\hline No & Yes & $P$ & No & $43.604431,3.736497$ \\
\hline No & Yes & $P$ & Yes & $43.618286,3.967411$ \\
\hline No & Yes & $P$ & No & 43.774444, 7.4975 \\
\hline No & Yes & $P$ & Yes & $43.124228,5.928$ \\
\hline No & Yes & $P$ & No & $44.250278,0.631942$ \\
\hline No & Yes & $P$ & No & $43.3,5.383333333$ \\
\hline
\end{tabular}




\begin{tabular}{|c|c|c|c|c|}
\hline No & Yes & $\mathrm{P}$ & No & $43.3,5.383333333$ \\
\hline No & Yes & $P$ & Yes & $43.553876,6.996755$ \\
\hline No & Yes & $P$ & Yes & $43.535682,6.449755$ \\
\hline No & Yes & $P$ & No & $43.721303,7.401067$ \\
\hline No & Yes & $?$ & No & $43.104102,6.073135$ \\
\hline No & Yes & $P$ & No & $43.923056,5.127778$ \\
\hline No & Yes & $\mathrm{P}$ & No & $43.618166,6.206349$ \\
\hline No & Yes & $P$ & No & $43.618166,6.206349$ \\
\hline No & Yes & $\mathrm{T}$ & No & $43.702457,4.666115$ \\
\hline No & Yes & $P$ & No & $43.763477,5.362582$ \\
\hline No & Yes & $P$ & No & $43.124228,5.928$ \\
\hline No & Yes & $P$ & No & $43.124228,5.928$ \\
\hline No & Yes & $P$ & No & $43.184277,3.003078$ \\
\hline No & Yes & $\mathrm{P} / \mathrm{T}$ & No & $43.610556,3.876667$ \\
\hline No & Yes & $\mathrm{T}$ & Yes & $43.123319,2.433942$ \\
\hline No & Yes & $P$ & No & $43.907481,7.155104$ \\
\hline No & Yes & $P$ & No & $43.710173,7.261953$ \\
\hline No & Yes & $\mathrm{P}$ & No & $43.610556,3.876667$ \\
\hline No & Yes & $P$ & No & $43.610556,3.876667$ \\
\hline No & No & n.a. & Yes & $43.314835,3.138366$ \\
\hline No & Yes & $?$ & No & $43.250631,5.405078$ \\
\hline No & Yes & $P$ & No & $43.515294,3.805496$ \\
\hline No & Yes & $\mathrm{P}$ & No & $43.184277,3.003078$ \\
\hline No & Yes & $P$ & No & $42.039604,9.012893$ \\
\hline No & Yes & $P$ & No & $43.610556,3.876667$ \\
\hline No & Yes & $P$ & Yes & $43.142984,5.916093$ \\
\hline No & No & n.a. & Yes & $44.098889,5.242222$ \\
\hline No & Yes & $\mathrm{P}$ & No & $43.710287,7.305119$ \\
\hline No & Yes & $\mathrm{P}$ & Yes & $43.774444,7.4975$ \\
\hline No & Yes & $P$ & Yes & $44.124667,5.214217$ \\
\hline No & Yes & $P$ & Yes & $43.774444,7.4975$ \\
\hline No & Yes & $P$ & Yes & $43.774444,7.4975$ \\
\hline No & Yes & $\mathrm{T}$ & No & $47.322047,5.04148$ \\
\hline No & Yes & $P$ & Yes & $43.393722,6.316657$ \\
\hline No & Yes & $P$ & Yes & $43.650664,1.55445$ \\
\hline No & Yes & $P$ & Yes & $43.529722,5.447222$ \\
\hline No & Yes & $\mathrm{T}$ & No & $42.529047,2.248169$ \\
\hline No & No & n.a. & Yes & $44.057113,5.19696$ \\
\hline Yes & Yes & $\mathrm{P}$ & Yes & // \\
\hline No & Yes & $\mathrm{P}$ & No & $43.783361,6.955305$ \\
\hline No & No & n.a. & Yes & $43.902293,5.292936$ \\
\hline No & Yes & $P$ & Yes & $43.949317,4.805528$ \\
\hline No & Yes & $P$ & Yes & $43.949317,4.805528$ \\
\hline No & Yes & $P$ & Yes & $44.01211,4.419946$ \\
\hline
\end{tabular}




\begin{tabular}{|c|c|c|c|c|}
\hline No & Yes & $\mathrm{P}$ & No & $44.056505,5.143207$ \\
\hline No & Yes & $P$ & No & $43.774444,7.4975$ \\
\hline Yes & No & n.a. & Yes & // \\
\hline No & Yes & $\mathrm{T}$ & No & $45.732448,4.88758$ \\
\hline No & Yes & $P$ & Yes & $43.646111,0.586667$ \\
\hline No & Yes & $P$ & Yes & $43.529722,5.447222$ \\
\hline No & Yes & $\mathrm{T}$ & Yes & $43.344167,3.215556$ \\
\hline No & Yes & $?$ & No & $43.296389,5.483333$ \\
\hline No & Yes & $P$ & No & $43.468969,3.811328$ \\
\hline No & Yes & $P$ & No & $43.660153,6.926492$ \\
\hline No & Yes & $P$ & No & $43.660153,6.926492$ \\
\hline No & Yes & $P$ & Yes & $43.660153,6.926492$ \\
\hline No & Yes & $\mathrm{T}$ & No & $43.657892,7.122337$ \\
\hline No & Yes & $P$ & No & $43.719923,6.979338$ \\
\hline No & Yes & $P$ & No & $43.698521,6.847956$ \\
\hline No & Yes & $P$ & No & $43.454229,-0.994301$ \\
\hline No & Yes & $P$ & Yes & $44.239147,5.126847$ \\
\hline No & Yes & $P$ & Yes & $43.616667,3.8833333$ \\
\hline No & Yes & $P$ & Yes & $43.627139,3.814167$ \\
\hline No & Yes & $P$ & Yes & $43.710287,7.305119$ \\
\hline No & Yes & $P$ & No & $43.3,5.383333333$ \\
\hline No & Yes & $P$ & Yes & $43.3,5.383333333$ \\
\hline No & Yes & $P$ & No & 43.3, 5.383333333 \\
\hline No & Yes & $\mathrm{T}$ & No & $44.014494,6.211644$ \\
\hline No & Yes & $\mathrm{T}$ & No & $46.160329,-1.151139$ \\
\hline No & Yes & $P$ & No & $42.688659,2.894833$ \\
\hline No & Yes & $P$ & No & $43.511944,3.883889$ \\
\hline No & Yes & $P$ & No & $42.501667,8.922778$ \\
\hline No & Yes & $P$ & No & $43.549722,3.778889$ \\
\hline No & Yes & $P$ & No & $43.625833,3.8225$ \\
\hline No & No & n.a. & Yes & // \\
\hline No & Yes & $\mathrm{T}$ & No & $43.646111,0.586667$ \\
\hline No & Yes & $P$ & Yes & 43.142984, 5.916093 \\
\hline No & Yes & $P$ & No & $43.610556,3.876667$ \\
\hline No & Yes & $P$ & No & $43.610556,3.876667$ \\
\hline No & Yes & $P$ & No & $43.610556,3.876667$ \\
\hline No & Yes & $P$ & Yes & $43.610556,3.876667$ \\
\hline No & Yes & $P$ & Yes & $45.648377,0.156237$ \\
\hline No & Yes & $P$ & Yes & $43.745833,0.7475$ \\
\hline No & Yes & $P$ & Yes & $43.646111,0.586667$ \\
\hline No & Yes & $P$ & Yes & $43.646111,0.586667$ \\
\hline No & Yes & $\mathrm{T}$ & No & $45.721944,-0.365$ \\
\hline Yes & Yes & $P$ & No & $/ /$ \\
\hline No & Yes & $\mathrm{T}$ & No & $46.160329,-1.151139$ \\
\hline
\end{tabular}




\begin{tabular}{|c|c|c|c|c|}
\hline No & Yes & $\mathrm{T}$ & No & $47.080833,2.398611$ \\
\hline No & Yes & $P$ & No & $43.610556,3.876667$ \\
\hline No & Yes & $P$ & No & $43.610556,3.876667$ \\
\hline No & Yes & $P$ & Yes & $43.393722,6.316657$ \\
\hline No & Yes & $P$ & Yes & $43.393722,6.316657$ \\
\hline No & Yes & $P$ & Yes & $43.710287,7.305119$ \\
\hline No & Yes & $\mathrm{P}$ & Yes & $43.710287,7.305119$ \\
\hline No & Yes & $\mathrm{P} / \mathrm{T}$ & Yes & $43.461389,3.423056$ \\
\hline No & Yes & $P$ & No & $43.580278,7.125$ \\
\hline No & Yes & $P$ & Yes & $43.646111,0.586667$ \\
\hline No & Yes & $P$ & Yes & $43.393722,6.316657$ \\
\hline No & Yes & $P$ & No & $43.662778,3.460556$ \\
\hline No & Yes & $P$ & No & $43.733611,3.313889$ \\
\hline No & Yes & $P$ & No & $43.384228,5.710687$ \\
\hline No & Yes & $P$ & Yes & $43.791551,3.732024$ \\
\hline No & Yes & $P$ & No & $43.124228,5.928$ \\
\hline No & Yes & $P$ & No & $43.407778,3.700556$ \\
\hline No & Yes & $P$ & No & $43.610556,3.876667$ \\
\hline No & Yes & $\mathrm{P}$ & Yes & $43.610556,3.876667$ \\
\hline No & No & n.a. & Yes & $44.098889,5.242222$ \\
\hline No & Yes & $P$ & Yes & $43.393722,6.316657$ \\
\hline No & Yes & $P$ & Yes & $43.393722,6.316657$ \\
\hline No & Yes & $P$ & No & $43.407778,3.700556$ \\
\hline No & Yes & $P$ & Yes & $41.422981,9.220775$ \\
\hline No & Yes & $P$ & No & $43.774444,7.4975$ \\
\hline No & Yes & $P$ & No & $43.604431,3.736497$ \\
\hline No & Yes & $P$ & No & // \\
\hline No & Yes & $P$ & No & $43.516111,3.694167$ \\
\hline No & Yes & $\mathrm{P}$ & Yes & $48.449572,-1.061482$ \\
\hline No & Yes & $P$ & Yes & $43.344167,3.215556$ \\
\hline No & Yes & $\mathrm{P}$ & No & $43.46,3.685278$ \\
\hline No & Yes & $P$ & No & $43.610556,3.876667$ \\
\hline No & Yes & $P$ & No & $43.625833,3.8225$ \\
\hline No & Yes & $P$ & No & $43.659167,3.816389$ \\
\hline No & Yes & $P$ & No & $43.684167,3.586667$ \\
\hline No & Yes & $P$ & No & $43.660153,6.926492$ \\
\hline No & Yes & $P$ & Yes & $43.529722,5.434722$ \\
\hline No & Yes & $P$ & Yes & $43.529722,5.447222$ \\
\hline No & Yes & $P$ & Yes & $45.648377,0.156237$ \\
\hline No & Yes & $P$ & No & $43.407778,3.700556$ \\
\hline No & Yes & $\mathrm{P}$ & No & $43.734167,3.548889$ \\
\hline No & No & n.a. & Yes & $45.648333,0.156111$ \\
\hline No & Yes & $\mathrm{P}$ & Yes & $43.636111,6.643333$ \\
\hline No & Yes & $P$ & No & $43.308611,3.476667$ \\
\hline
\end{tabular}




\begin{tabular}{|c|c|c|c|c|}
\hline No & Yes & $P$ & No & $43.610556,3.876667$ \\
\hline No & Yes & $P$ & No & $44.361667,5.14$ \\
\hline No & Yes & $P$ & No & $44.361667,5.14$ \\
\hline Yes & Yes & n.a. & No & // \\
\hline Yes & Yes & $\mathrm{T}$ & Yes & $/ /$ \\
\hline No & Yes & $P$ & No & $43.021111,3.043889$ \\
\hline No & Yes & $P$ & No & $43.021111,3.043889$ \\
\hline No & Yes & $P$ & Yes & $44.924167,-0.271111$ \\
\hline No & Yes & $P$ & No & $43.021111,3.043889$ \\
\hline No & Yes & $P$ & No & $41.387174,9.159269$ \\
\hline No & Yes & $P$ & No & $43.021111,3.043889$ \\
\hline No & Yes & $P$ & No & $41.387174,9.159269$ \\
\hline No & Yes & $P$ & No & $45.648377,0.156237$ \\
\hline No & Yes & $P$ & Yes & 41.386944, 9.159167 \\
\hline No & Yes & $\mathrm{P}$ & No & $44.100556,3.077778$ \\
\hline Yes & No & n.a. & Yes & // \\
\hline No & Yes & $\mathrm{P}$ & No & $43.803006,3.755055$ \\
\hline No & Yes & $P$ & Yes & $43.791975,3.739444$ \\
\hline No & Yes & $\mathrm{P}$ & Yes & $43.433056,6.736944$ \\
\hline No & Yes & $\mathrm{P} / \mathrm{T}$ & No & $43.368611,3.157222$ \\
\hline No & Yes & $\mathrm{P}$ & No & $43.344167,3.215556$ \\
\hline No & Yes & $P$ & No & $43.296389,5.483333$ \\
\hline No & Yes & $\mathrm{T}$ & No & $47.3475,0.548333$ \\
\hline No & Yes & $\mathrm{P}$ & No & $44.124667,5.214217$ \\
\hline No & No & n.a. & Yes & $43.629206,3.865351$ \\
\hline No & Yes & $\mathrm{P}$ & No & $43.629206,3.865351$ \\
\hline No & Yes & $P$ & Yes & 43.632037, 3.891778 \\
\hline No & Yes & $P$ & No & $43.660153,6.926492$ \\
\hline No & Yes & $P$ & No & $43.645052,3.876418$ \\
\hline No & Yes & $P$ & No & $43.604431,3.736497$ \\
\hline No & Yes & $P$ & No & $44.124667,5.214217$ \\
\hline No & Yes & $\mathrm{T}$ & No & $44.819444,-0.550556$ \\
\hline No & Yes & $P$ & No & // \\
\hline No & Yes & $P$ & No & $42.688659,2.894833$ \\
\hline No & Yes & $P$ & No & $42.889167,2.718333$ \\
\hline No & Yes & $P$ & No & $43.344167,3.215556$ \\
\hline No & Yes & $P$ & No & $43.344167,3.215556$ \\
\hline No & Yes & $\mathrm{P} / \mathrm{T}$ & Yes & $43.521389,2.816917$ \\
\hline No & Yes & $\mathrm{T}$ & No & $43.529722,5.447222$ \\
\hline No & Yes & $\mathrm{T}$ & No & $43.783333,4.089722$ \\
\hline No & Yes & $P$ & No & $47.836111,6.096389$ \\
\hline Yes & Yes & $\mathrm{T}$ & No & // \\
\hline No & Yes & $P$ & No & $43.142984,5.916093$ \\
\hline No & Yes & $\mathrm{P}$ & No & 43.139901, 5.847652 \\
\hline
\end{tabular}




\begin{tabular}{|c|c|c|c|c|}
\hline No & Yes & $\mathrm{P}$ & No & $43.836699,4.360054$ \\
\hline No & Yes & $\mathrm{P}$ & Yes & $43.610556,3.876667$ \\
\hline Yes & Yes & $\mathrm{T}$ & Yes & // \\
\hline No & Yes & $P$ & No & $44.014494,6.211644$ \\
\hline Yes & Yes & $\mathrm{P} / \mathrm{T}$ & No & // \\
\hline No & Yes & $\mathrm{T}$ & No & $48.900552,2.25929$ \\
\hline No & No & n.a. & Yes & // \\
\hline No & Yes & $\mathrm{P}$ & No & $43.610556,3.876667$ \\
\hline No & Yes & $P$ & No & $43.763477,5.362582$ \\
\hline No & Yes & $P$ & No & $43.763477,5.362582$ \\
\hline No & Yes & $\mathrm{P}$ & Yes & $43.610556,3.876667$ \\
\hline No & Yes & $\mathrm{P} / \mathrm{T}$ & Yes & $43.660153,6.926492$ \\
\hline No & Yes & $\mathrm{P}$ & No & $43.660153,6.926492$ \\
\hline Yes & Yes & $\mathrm{T}$ & No & // \\
\hline No & No & n.a. & No & $45.648377,0.156237$ \\
\hline No & Yes & $\mathrm{P}$ & No & $43.646111,0.586667$ \\
\hline No & Yes & $P$ & Yes & // \\
\hline No & Yes & $\mathrm{P}$ & No & $42.712812,2.950954$ \\
\hline No & No & n.a. & No & $43.610556,3.876667$ \\
\hline No & No & n.a. & No & $44.658341,0.060664$ \\
\hline No & Yes & n.a. & No & $43.610556,3.876667$ \\
\hline No & Yes & $\mathrm{P}$ & No & $45.751996,0.153476$ \\
\hline No & Yes & $P$ & Yes & $45.648377,0.156237$ \\
\hline No & Yes & $P$ & No & $43.610556,3.876667$ \\
\hline No & Yes & $P$ & No & $43.124228,5.928$ \\
\hline No & Yes & $P$ & No & $43.610556,3.876667$ \\
\hline No & Yes & $P$ & No & $43.610556,3.876667$ \\
\hline No & Yes & $P$ & Yes & $43.646111,0.586667$ \\
\hline No & No & n.a. & Yes & $43.142984,5.916093$ \\
\hline No & No & n.a. & Yes & $43.142984,5.916093$ \\
\hline No & Yes & $P$ & No & $41.387174,9.159269$ \\
\hline No & Yes & $P$ & Yes & $45.487706,0.355149$ \\
\hline No & Yes & $P$ & Yes & $43.184277,3.003078$ \\
\hline No & Yes & $\mathrm{T}$ & Yes & $43.836699,4.360054$ \\
\hline No & Yes & $P$ & No & $45.648377,0.156237$ \\
\hline No & Yes & $P$ & No & $43.652984,3.508645$ \\
\hline No & Yes & $P$ & No & $43.610556,3.876667$ \\
\hline No & Yes & $P$ & Yes & $43.610556,3.876667$ \\
\hline No & Yes & $P$ & No & $43.610556,3.876667$ \\
\hline No & Yes & $P$ & No & $43.610556,3.876667$ \\
\hline No & Yes & $P$ & No & 43.142984, 5.916093 \\
\hline No & Yes & $P$ & No & $43.142984,5.916093$ \\
\hline No & Yes & $P$ & No & $43.142984,5.916093$ \\
\hline No & Yes & $P$ & No & $43.142984,5.916093$ \\
\hline
\end{tabular}




\begin{tabular}{|c|c|c|c|c|}
\hline No & Yes & $P$ & No & $43.646111,0.586667$ \\
\hline No & Yes & $P$ & No & $43.646111,0.586667$ \\
\hline No & Yes & $P$ & No & $43.646111,0.586667$ \\
\hline No & No & n.a. & Yes & $44.203142,0.616363$ \\
\hline No & No & $\mathrm{P}$ & No & $44.284151,1.457949$ \\
\hline No & Yes & $P$ & No & $45.648377,0.156237$ \\
\hline No & Yes & $P$ & No & $43.610556,3.876667$ \\
\hline No & Yes & $P$ & No & $43.610556,3.876667$ \\
\hline No & Yes & $?$ & No & $48.804689,2.289387$ \\
\hline No & Yes & $P$ & No & $46.160329,-1.151139$ \\
\hline No & Yes & $P$ & Yes & $46.572727,-0.964886$ \\
\hline No & Yes & $P$ & No & $46.572727,-0.964886$ \\
\hline No & Yes & $P$ & No & $44.255647,0.629892$ \\
\hline No & Yes & $P$ & No & $45.744161,-0.631667$ \\
\hline No & Yes & $\mathrm{T}$ & No & $43.646111,0.586667$ \\
\hline No & Yes & $\mathrm{T}$ & Yes & $43.646111,0.586667$ \\
\hline No & No & n.a. & Yes & $42.692003,9.450392$ \\
\hline No & Yes & $\mathrm{P}$ & No & $46.454772,-1.167062$ \\
\hline Yes & Yes & $P$ & No & $/ /$ \\
\hline Yes & Yes & $\mathrm{T}$ & Yes & // \\
\hline No & Yes & $P$ & No & $43.124228,5.928$ \\
\hline No & Yes & $\mathrm{P}$ & No & $43.53324,3.861616$ \\
\hline No & No & n.a. & Yes & $43.234312,5.361918$ \\
\hline No & No & n.a. & Yes & $43.234312,5.361918$ \\
\hline No & Yes & $\mathrm{P}$ & Yes & $43.393722,6.316657$ \\
\hline No & Yes & $P$ & Yes & $43.393722,6.316657$ \\
\hline No & Yes & $P$ & Yes & $43.393722,6.316657$ \\
\hline No & Yes & $P$ & No & $43.341893,2.361444$ \\
\hline No & Yes & $P$ & No & $43.341893,2.361444$ \\
\hline No & Yes & $P$ & No & $43.580278,7.125$ \\
\hline No & Yes & $P$ & No & $43.234312,5.361918$ \\
\hline No & Yes & $P$ & No & $43.393722,6.316657$ \\
\hline No & Yes & $P$ & No & $43.393722,6.316657$ \\
\hline No & Yes & $P$ & Yes & $43.393722,6.316657$ \\
\hline No & Yes & $\mathrm{P}$ & No & $43.234312,5.361918$ \\
\hline No & Yes & $\mathrm{P} / \mathrm{T}$ & No & $44.392471,6.628415$ \\
\hline No & Yes & $\mathrm{P}$ & No & $43.124228,5.928$ \\
\hline No & Yes & $P$ & No & $43.710173,7.261953$ \\
\hline No & Yes & $\mathrm{P}$ & No & $43.710173,7.261953$ \\
\hline No & Yes & $P$ & No & $43.710173,7.261953$ \\
\hline No & Yes & $P$ & Yes & $43.710173,7.261953$ \\
\hline No & Yes & $P$ & Yes & $43.710173,7.261953$ \\
\hline No & Yes & $P$ & Yes & $43.710287,7.305119$ \\
\hline No & Yes & $P$ & Yes & $43.710287,7.305119$ \\
\hline
\end{tabular}




\begin{tabular}{|c|c|c|c|c|}
\hline No & Yes & $P$ & No & 43.710287, 7.305119 \\
\hline No & Yes & $P$ & No & $43.710287,7.305119$ \\
\hline No & Yes & $P$ & Yes & $43.710287,7.305119$ \\
\hline No & Yes & $P$ & No & $43.710287,7.305119$ \\
\hline No & Yes & $P$ & No & $43.344167,3.215556$ \\
\hline No & Yes & $P$ & Yes & $43.710287,7.305119$ \\
\hline No & Yes & $P$ & No & $42.768583,2.871763$ \\
\hline No & Yes & $\mathrm{T}$ & Yes & $44.461111,1.902778$ \\
\hline No & Yes & $P$ & No & $43.836605,5.040781$ \\
\hline No & Yes & $P$ & No & $43.834552,5.034722$ \\
\hline No & Yes & $P$ & No & $43.647718,3.797514$ \\
\hline No & Yes & $P$ & No & 43.519925, 7.0485 \\
\hline No & Yes & $P$ & No & 43.774444, 7.4975 \\
\hline No & Yes & n.a. & No & $49.196095,2.914006$ \\
\hline No & Yes & $\mathrm{P}$ & Yes & $44.943275,1.006074$ \\
\hline No & Yes & $P$ & No & // \\
\hline No & Yes & $P$ & No & $43.159943,5.617941$ \\
\hline Yes & Yes & $\mathrm{T}$ & No & // \\
\hline No & Yes & $P$ & No & $44.056505,5.143207$ \\
\hline No & Yes & $P$ & No & $43.710173,7.261953$ \\
\hline Yes & Yes & $\mathrm{T}$ & No & // \\
\hline No & Yes & $P$ & No & $42.692003,9.450392$ \\
\hline No & Yes & $P$ & No & $48.267043,2.692611$ \\
\hline Yes & Yes & $\mathrm{T}$ & Yes & // \\
\hline No & Yes & $P$ & No & $44.203142,0.616363$ \\
\hline No & Yes & $\mathrm{T}$ & No & $44.728275,5.024072$ \\
\hline No & Yes & $\mathrm{T}$ & No & $44.728275,5.024072$ \\
\hline No & Yes & $?$ & No & $48.533333,2.8$ \\
\hline No & Yes & $P$ & No & $44.138099,4.807511$ \\
\hline No & Yes & $P$ & No & $46.572727,-0.964886$ \\
\hline No & Yes & $P$ & No & $47.49973,1.46013$ \\
\hline No & Yes & $P$ & No & $44.056505,5.143207$ \\
\hline No & Yes & $\mathrm{T}$ & No & $44.900794,4.802146$ \\
\hline No & Yes & $\mathrm{P}$ & No & $43.124228,5.928$ \\
\hline No & Yes & n.a. & No & $43.407778,3.700556$ \\
\hline No & Yes & $\mathrm{P}$ & No & $43.891711,3.632081$ \\
\hline No & Yes & $P$ & Yes & $43.610556,3.876667$ \\
\hline No & Yes & $\mathrm{P}$ & Yes & $46.454772,-1.167062$ \\
\hline No & Yes & $\mathrm{P} / \mathrm{T}$ & Yes & $43.393722,6.316657$ \\
\hline No & Yes & $\mathrm{T}$ & No & $45.777222,3.087025$ \\
\hline No & Yes & n.a. & No & $43.393722,6.316657$ \\
\hline No & Yes & $P$ & No & $43.393722,6.316657$ \\
\hline No & Yes & $P$ & Yes & $43.393722,6.316657$ \\
\hline No & Yes & $P$ & No & $43.393722,6.316657$ \\
\hline
\end{tabular}




\begin{tabular}{|c|c|c|c|c|}
\hline No & Yes & $P$ & No & $44.098889,5.242222$ \\
\hline No & Yes & $P$ & No & $43.650664,1.55445$ \\
\hline No & Yes & $P$ & No & $43.661506,3.341121$ \\
\hline No & Yes & $P$ & No & $43.650664,1.55445$ \\
\hline No & Yes & $P$ & No & $43.650664,1.55445$ \\
\hline No & Yes & $P$ & No & $43.650664,1.55445$ \\
\hline No & Yes & $\mathrm{P} / \mathrm{T}$ & No & $44.100556,3.077778$ \\
\hline No & Yes & $\mathrm{T}$ & Yes & $44.504031,0.929523$ \\
\hline No & Yes & $P$ & No & 41.422981, 9.220775 \\
\hline No & Yes & $P$ & No & 41.422981, 9.220775 \\
\hline No & Yes & $P$ & Yes & $41.422981,9.220775$ \\
\hline No & Yes & $P$ & No & $41.421069,9.184851$ \\
\hline No & Yes & $P$ & No & $43.754365,7.320831$ \\
\hline Yes & No & n.a. & Yes & $41.238987,9.188079$ \\
\hline Yes & Yes & $\mathrm{P}$ & No & $41.238987,9.188079$ \\
\hline Yes & Yes & $\mathrm{T}$ & No & // \\
\hline No & No & n.a. & Yes & $43.610556,3.876667$ \\
\hline No & Yes & $\mathrm{P}$ & No & $43.282801,3.279651$ \\
\hline No & Yes & $P$ & No & $44.002722,7.554131$ \\
\hline No & Yes & $P$ & Yes & $44.002722,7.554131$ \\
\hline No & Yes & $P$ & No & // \\
\hline No & Yes & $\mathrm{T}$ & Yes & $47.336421,-3.181043$ \\
\hline Yes & Yes & $P$ & Yes & // \\
\hline Yes & Yes & $P$ & Yes & // \\
\hline Yes & Yes & $P$ & Yes & $/ /$ \\
\hline No & Yes & $P$ & No & $43.212161,2.353663$ \\
\hline No & Yes & $\mathrm{T}$ & Yes & $45.899247,6.129384$ \\
\hline Yes & Yes & $\mathrm{T}$ & Yes & // \\
\hline No & Yes & n.a. & No & $43.610556,3.876667$ \\
\hline No & Yes & $P$ & No & $43.529722,5.447222$ \\
\hline No & Yes & $P$ & Yes & $43.529722,5.447222$ \\
\hline No & Yes & $P$ & No & $43.529722,5.447222$ \\
\hline No & Yes & $P$ & Yes & $43.529722,5.447222$ \\
\hline No & Yes & $P$ & No & $46.147551,-1.210261$ \\
\hline No & Yes & $P$ & No & $43.689829,7.301271$ \\
\hline Yes & Yes & $\mathrm{T}$ & No & // \\
\hline No & Yes & $P$ & No & $43.923056,5.127778$ \\
\hline No & Yes & $\mathrm{P}$ & No & $43.710173,7.261953$ \\
\hline No & No & n.a. & No & $43.106848,-0.673751$ \\
\hline No & Yes & $P$ & No & $43.580278,7.125$ \\
\hline No & Yes & $\mathrm{T}$ & No & $48.306124,-0.620935$ \\
\hline No & Yes & $P$ & No & $46.364005,5.346603$ \\
\hline No & Yes & $P$ & No & $42.104248,9.512429$ \\
\hline No & Yes & $P$ & No & $44.924167,-0.271111$ \\
\hline
\end{tabular}




\begin{tabular}{|c|c|c|c|c|}
\hline No & Yes & $P$ & No & $43.021111,3.043889$ \\
\hline No & Yes & $\mathrm{T}$ & No & $44.09216,2.999246$ \\
\hline No & Yes & $\mathrm{T}$ & No & $48.443854,1.489012$ \\
\hline No & No & n.a. & No & $44.924167,-0.271111$ \\
\hline No & Yes & n.a. & No & // \\
\hline No & Yes & $\mathrm{P} / \mathrm{T}$ & No & $44.100556,3.077778$ \\
\hline No & Yes & $\mathrm{P}$ & No & 41.387174, 9.159269 \\
\hline No & Yes & $P$ & No & $41.387174,9.159269$ \\
\hline No & Yes & $P$ & No & $41.387174,9.159269$ \\
\hline No & Yes & $P$ & No & $43.021111,3.043889$ \\
\hline No & Yes & $P$ & No & $45.648377,0.156237$ \\
\hline No & Yes & $P$ & No & $45.146949,0.757221$ \\
\hline No & Yes & $P$ & No & $43.021111,3.043889$ \\
\hline No & Yes & $P$ & No & $43.660153,6.926492$ \\
\hline No & Yes & $\mathrm{T}$ & No & $48.565556,2.06$ \\
\hline No & Yes & $\mathrm{T}$ & No & $48.565556,2.06$ \\
\hline No & Yes & $P$ & No & $43.602332,7.006491$ \\
\hline No & Yes & $\mathrm{P} / \mathrm{T}$ & No & $43.407778,3.700556$ \\
\hline No & Yes & $\mathrm{P}$ & Yes & $43.296482,5.36978$ \\
\hline No & Yes & $\mathrm{T}$ & No & $43.232951,0.078082$ \\
\hline Yes & Yes & $\mathrm{T}$ & Yes & // \\
\hline Yes & Yes & $\mathrm{T}$ & Yes & $/ /$ \\
\hline No & Yes & $\mathrm{T}$ & No & $43.407778,3.700556$ \\
\hline No & Yes & $\mathrm{T}$ & No & $48.970782,2.256869$ \\
\hline No & Yes & $\mathrm{P}$ & No & $43.610556,3.876667$ \\
\hline No & No & n.a. & No & $48.846066,2.188703$ \\
\hline No & Yes & $\mathrm{P}$ & No & $43.267681,6.640711$ \\
\hline No & Yes & $P$ & No & $43.646111,0.586667$ \\
\hline No & Yes & $P$ & No & $43.326263,3.377885$ \\
\hline No & No & n.a. & Yes & $43.267681,6.640711$ \\
\hline No & No & n.a. & No & $43.267681,6.640711$ \\
\hline No & Yes & $\mathrm{P}$ & No & $43.267681,6.640711$ \\
\hline No & Yes & $\mathrm{T}$ & No & $43.677079,4.433703$ \\
\hline No & No & n.a. & No & $48.985761,2.350813$ \\
\hline No & Yes & $\mathrm{T}$ & No & $49.038069,2.360491$ \\
\hline No & Yes & $P$ & No & $43.344167,3.215556$ \\
\hline No & Yes & $P$ & No & $43.344167,3.215556$ \\
\hline No & Yes & $\mathrm{T}$ & Yes & $46.433063,-0.853614$ \\
\hline No & Yes & n.a. & Yes & $43.722322,7.111703$ \\
\hline No & Yes & $\mathrm{T}$ & No & $48.81833,-3.473795$ \\
\hline No & Yes & $\mathrm{P}$ & No & $43.40655,6.061187$ \\
\hline No & No & n.a. & No & $48.801408,2.130122$ \\
\hline Yes & No & n.a. & No & // \\
\hline No & No & n.a. & No & $43.710173,7.261953$ \\
\hline
\end{tabular}




\begin{tabular}{|c|c|c|c|c|}
\hline No & Yes & $P$ & Yes & $43.125556,6.13$ \\
\hline No & Yes & $\mathrm{T}$ & No & $43.96341,4.796959$ \\
\hline No & Yes & $P$ & No & $43.710173,7.261953$ \\
\hline No & Yes & $\mathrm{P}$ & No & $43.911315,5.200176$ \\
\hline No & No & n.a. & No & $45.036291,1.037587$ \\
\hline No & Yes & $\mathrm{T}$ & No & $45.036291,1.037587$ \\
\hline No & Yes & $P$ & No & // \\
\hline No & Yes & $P$ & No & $43.911315,5.200176$ \\
\hline No & Yes & $P$ & No & $43.296389,5.483333$ \\
\hline No & Yes & $P$ & No & $43.296389,5.483333$ \\
\hline No & Yes & $P$ & No & $43.296389,5.483333$ \\
\hline No & Yes & $\mathrm{T}$ & No & $43.136418,5.754186$ \\
\hline No & No & n.a. & Yes & $43.124228,5.928$ \\
\hline No & Yes & $\mathrm{P}$ & Yes & $43.75299,7.381835$ \\
\hline No & Yes & $P$ & No & $43.124228,5.928$ \\
\hline No & No & n.a. & Yes & $45.501124,4.44043$ \\
\hline No & Yes & $\mathrm{P}$ & No & $43.334817,5.756087$ \\
\hline No & Yes & $\mathrm{P}$ & No & $43.610556,3.876667$ \\
\hline No & No & n.a. & Yes & $45.766714,1.167562$ \\
\hline No & Yes & $\mathrm{P}$ & No & $43.72208,7.405839$ \\
\hline No & Yes & $\mathrm{T}$ & No & $45.744161,-0.631667$ \\
\hline No & No & n.a. & No & $43.610556,3.876667$ \\
\hline No & Yes & $P$ & No & $43.610556,3.876667$ \\
\hline No & Yes & $P$ & No & $43.610556,3.876667$ \\
\hline No & Yes & $P$ & No & $45.648377,0.156237$ \\
\hline No & Yes & $P$ & No & 43.438959, 6.071791 \\
\hline No & Yes & $P$ & No & $43.559215,5.393301$ \\
\hline No & Yes & n.a. & No & $48.749736,2.459965$ \\
\hline No & Yes & $\mathrm{T}$ & No & $48.770878,2.522163$ \\
\hline Yes & Yes & $\mathrm{T}$ & No & // \\
\hline Yes & Yes & $\mathrm{T}$ & Yes & // \\
\hline No & Yes & $\mathrm{T}$ & No & $48.556855,2.550114$ \\
\hline No & Yes & $P$ & No & $43.664817,5.155542$ \\
\hline No & Yes & $P$ & No & $43.555284,5.602142$ \\
\hline No & Yes & $P$ & No & $45.655485,0.161095$ \\
\hline No & Yes & $P$ & No & $45.864332,-0.506285$ \\
\hline No & Yes & $P$ & No & $45.864332,-0.506285$ \\
\hline No & Yes & $P$ & No & $45.864332,-0.506285$ \\
\hline No & Yes & $P$ & No & $43.683448,3.840085$ \\
\hline Yes & No & n.a. & No & // \\
\hline No & Yes & $P$ & Yes & $44.124667,5.214217$ \\
\hline Yes & No & n.a. & Yes & // \\
\hline No & Yes & $\mathrm{P}$ & No & $45.61349,0.18178$ \\
\hline No & Yes & $\mathrm{P}$ & No & $45.61349,0.18178$ \\
\hline
\end{tabular}




\begin{tabular}{|c|c|c|c|c|}
\hline No & Yes & $P$ & No & $43.660153,6.926492$ \\
\hline No & Yes & $P$ & No & $43.660153,6.926492$ \\
\hline No & Yes & n.a. & No & 48.709298, 2.38479 \\
\hline No & Yes & $\mathrm{T}$ & No & $48.709298,2.38479$ \\
\hline Yes & Yes & $\mathrm{T}$ & No & // \\
\hline No & Yes & $P$ & No & $44.393179,4.753137$ \\
\hline No & Yes & $P$ & No & $44.393179,4.753137$ \\
\hline No & No & n.a. & No & $43.875204,6.512101$ \\
\hline No & Yes & $\mathrm{P}$ & No & $43.783361,6.955305$ \\
\hline No & Yes & $P$ & No & $44.239147,5.126847$ \\
\hline Yes & Yes & $\mathrm{T}$ & No & // \\
\hline Yes & Yes & $\mathrm{T}$ & No & $/ /$ \\
\hline Yes & No & $\mathrm{T}$ & Yes & $/ /$ \\
\hline Yes & No & $\mathrm{T}$ & Yes & $/ /$ \\
\hline Yes & Yes & $P$ & No & $/ /$ \\
\hline Yes & Yes & $P$ & No & $/ /$ \\
\hline Yes & Yes & $P$ & No & $/ /$ \\
\hline Yes & Yes & $\mathrm{T}$ & No & $/ /$ \\
\hline Yes & Yes & $\mathrm{T}$ & No & $/ /$ \\
\hline Yes & Yes & $\mathrm{T}$ & No & $/ /$ \\
\hline Yes & Yes & $\mathrm{T}$ & No & $/ /$ \\
\hline No & Yes & $\mathrm{P} / \mathrm{T}$ & No & // \\
\hline No & Yes & $\mathrm{P}$ & Yes & $43.2951,-0.370797$ \\
\hline No & Yes & n.a. & No & $43.710173,7.261953$ \\
\hline No & No & n.a. & Yes & $43.610556,3.876667$ \\
\hline No & No & n.a. & Yes & $47.903352,1.234261$ \\
\hline No & No & n.a. & No & $43.102976,5.878219$ \\
\hline No & No & n.a. & Yes & // \\
\hline No & Yes & $\mathrm{P}$ & No & $45.487706,0.355149$ \\
\hline No & Yes & $P$ & No & $47.375865,3.974876$ \\
\hline No & Yes & $P$ & Yes & 41.387174, 9.159269 \\
\hline No & Yes & $P$ & No & $43.610556,3.876667$ \\
\hline No & Yes & $P$ & No & $43.184277,3.003078$ \\
\hline No & Yes & $P$ & No & $43.124665,6.125104$ \\
\hline No & Yes & $P$ & No & $43.710173,7.261953$ \\
\hline No & Yes & $P$ & No & $43.610556,3.876667$ \\
\hline No & Yes & $P$ & No & // \\
\hline No & Yes & $P$ & No & $45.744161,-0.631667$ \\
\hline No & Yes & $P$ & No & $42.688659,2.894833$ \\
\hline No & Yes & $P$ & No & 41.387174, 9.159269 \\
\hline No & Yes & $\mathrm{T}$ & No & $43.949317,4.805528$ \\
\hline No & Yes & $\mathrm{T}$ & No & $46.311555,3.416765$ \\
\hline No & Yes & $\mathrm{T}$ & No & $46.454772,-1.167062$ \\
\hline No & Yes & $\mathrm{T}$ & No & $48.117266,-1.677793$ \\
\hline
\end{tabular}




\begin{tabular}{|c|c|c|c|c|}
\hline Yes & Yes & $P$ & Yes & $/ /$ \\
\hline Yes & Yes & $\mathrm{T}$ & No & $/ /$ \\
\hline No & Yes & $\mathrm{T}$ & Yes & $44.461111,1.902778$ \\
\hline No & No & n.a. & Yes & $44.461111,1.902778$ \\
\hline No & Yes & $\mathrm{P}$ & Yes & $41.422981,9.220775$ \\
\hline No & Yes & $P$ & Yes & $46.454772,-1.167062$ \\
\hline No & Yes & $\mathrm{T}$ & No & 43.774444, 7.4975 \\
\hline No & Yes & $P$ & Yes & $43.529722,5.447222$ \\
\hline No & Yes & $P$ & No & $43.598064,5.445938$ \\
\hline No & Yes & $P$ & Yes & 43.774444, 7.4975 \\
\hline No & Yes & $\mathrm{T}$ & No & $47.875568,-4.106373$ \\
\hline No & Yes & $\mathrm{T}$ & No & $47.875568,-4.106373$ \\
\hline No & Yes & $P$ & Yes & $43.646111,0.586667$ \\
\hline No & Yes & $P$ & No & $43.610556,3.876667$ \\
\hline No & No & n.a. & Yes & $43.710173,7.261953$ \\
\hline No & Yes & $\mathrm{P}$ & No & $43.700818,7.300177$ \\
\hline No & Yes & $P$ & No & $43.296482,5.369780$ \\
\hline No & No & n.a. & No & $43.717533,7.295514$ \\
\hline No & Yes & $P$ & Yes & $41.406445,9.169541$ \\
\hline No & Yes & $P$ & No & $43.296482,5.36978$ \\
\hline No & Yes & $P$ & No & $43.610556,3.876667$ \\
\hline No & Yes & $P$ & Yes & $43.124228,5.928$ \\
\hline No & Yes & $?$ & Yes & $43.344167,3.215556$ \\
\hline No & Yes & $\mathrm{P} / \mathrm{T}$ & No & $43.404811,5.053728$ \\
\hline No & Yes & $\mathrm{P}$ & Yes & $46.454772,-1.167062$ \\
\hline No & Yes & $P$ & Yes & 43.774444, 7.4975 \\
\hline No & Yes & $P$ & Yes & $44.002722,7.554131$ \\
\hline No & Yes & $P$ & No & 43.774444, 7.4975 \\
\hline No & Yes & $P$ & Yes & $43.752541,3.955276$ \\
\hline No & Yes & $P$ & Yes & $43.646111,0.586667$ \\
\hline No & Yes & $P$ & Yes & $43.646111,0.586667$ \\
\hline No & Yes & $P$ & Yes & $43.646111,0.586667$ \\
\hline No & Yes & $\mathrm{T}$ & Yes & $45.988917,4.696849$ \\
\hline No & No & n.a. & Yes & $46.572727,-0.964886$ \\
\hline No & Yes & $\mathrm{P}$ & No & $43.393722,6.316657$ \\
\hline No & Yes & $\mathrm{P}$ & No & $47.384882,0.554293$ \\
\hline No & No & n.a. & No & $43.610556,3.876667$ \\
\hline No & Yes & $\mathrm{P}$ & No & $43.559215,5.393301$ \\
\hline No & No & n.a. & Yes & $43.393722,6.316657$ \\
\hline No & Yes & $\mathrm{P}$ & No & $42.682090,2.899657$ \\
\hline
\end{tabular}




\begin{tabular}{|c|c|c|c|c|c|c|}
\hline \multirow[t]{2}{*}{ Herbarium } & \multicolumn{2}{|c|}{ Barcode or referen Collector } & \multicolumn{2}{|c|}{ Collection nu } & \multicolumn{2}{|c|}{ Collection date } \\
\hline & & & & Day & Month & Year \\
\hline B & В 100525364 & F. Schultz & 408 & 26 & 5 & 1849 \\
\hline B & В 100525361 & Canut & 391 & 29 & 5 & 1862 \\
\hline B & В 100525360 & leg. ign. & 391 & n.a. & 6 & 1896 \\
\hline B & В 100525359 & H.L. Krause & 30549 & 9 & 7 & 1910 \\
\hline BC & 135239 & leg. ign. & s.n. & 20 & 5 & 1865 \\
\hline BC & $833041-2$ & Sennen & s.n. & 27 & 4 & 1899 \\
\hline BC & $833040-2$ & C.-A Gérard & s.n. & 12 & 6 & 1901 \\
\hline BC & 1523 & Marnac & s.n. & 19 & 6 & 1911 \\
\hline BC & 1543 & L. Samat & 6059 & 25 & 5 & 1930 \\
\hline BC & $373730-3$ & G. Gavelli & s.n. & 5 & 6 & 1963 \\
\hline $\mathrm{BC}$ & 135237 & Gerclard & s.n. & 4 & 9 & 1980 \\
\hline BM & BM001209454 & J.M. Delalande & s.n. & n.a. & 9 & 1848 \\
\hline BM & BM001209450 & M. Prost & s.n. & n.a. & 6 & 1849 \\
\hline BM & BM000984476 & J. Revellière & 8 & 14 & 6 & 1854 \\
\hline BM & BM001209512 & J.C. Ducommun & s.n. & 26 & 5 & 1859 \\
\hline BM & BM001209455 & F.S.A. Augé de La & ss.n. & 10 & 6 & 1860 \\
\hline BM & BM001209509 & E. Miciol & s.n. & 4 & 6 & 1864 \\
\hline BM & BM001209509 & E. Miciol & s.n. & 4 & 6 & 1864 \\
\hline BM & BM000649099 & [illegible] & s.n. & 30 & 6 & 1867 \\
\hline BM & BM000984501 & P.A. Kesselmeye & s.n. & n.a. & 6 & 1867 \\
\hline BM & BM000984475 & J-O. Debeaux & s.n. & 30 & 5 & 1869 \\
\hline BM & BM000984475 & J-O. Debeaux & s.n. & 30 & 5 & 1869 \\
\hline BM & BM001209453 & E.J. de Puyfol & s.n. & 13 & 5 & 1874 \\
\hline BM & BM001209451 & L. Anthouard & s.n. & 9 & 6 & 1875 \\
\hline BM & BM001209452 & A. Cartier & 1487 & 20 & 5 & 1877 \\
\hline BM & BM001209505 & E. Marçais & s.n. & n.a. & $6--7$ & 1878 \\
\hline BM & BM001209412 & E. Reverchon & s.n. & 14 & 6 & 1880 \\
\hline BM & BM001209507 & N.D. Simpson & 36543 & n.a. & 5 & 1883 \\
\hline BM & BM000984488 & J. Bruneau & s.n. & 19 & 6 & 1889 \\
\hline BM & BM001209508 & J. Lloyd & s.n. & 20 & 4 & 1891 \\
\hline BM & BM001209510 & E.J.A. Gadeceau & s.n. & 2 & 6 & 1893 \\
\hline BM & BM001209514 & V. Bonafons & s.n. & n.a. & 6 & 1894 \\
\hline BM & BM001209506 & Septimin-Donat & 111 & 8 & 6 & 1898 \\
\hline BM & BM000649100 & P.R. Lowe & s.n. & 22 & 5 & 1911 \\
\hline BM & BM000649110 & J. Roffey & s.n. & 15 & 6 & 1925 \\
\hline BM & BM000649076 & P.J.R. Bamps & 9002 & 12 & 6 & 1991 \\
\hline E & E00743450 & G.W. Arnott & s.n. & 18 & 5 & 1825 \\
\hline$E$ & E00743402 & Seringe & 7 & 18 & 5 & 1825 \\
\hline$E$ & E00743403 & Seringe & 127 & 22 & 5 & 1825 \\
\hline$E$ & E00743439 & F. Kuyes (?) & s.n. & n.a. & 6 & 1839 \\
\hline$E$ & E00743413 & F. Rugel & s.n. & n.a. & 6 & 1839 \\
\hline$E$ & E00743413 & F. Rugel & s.n. & n.a. & 5 & 1839 \\
\hline
\end{tabular}




\begin{tabular}{|c|c|c|c|c|c|c|}
\hline$E$ & E00445049 & leg. ign. & s.n. & n.a. & 6 & 1855 \\
\hline$E$ & E00743421 & J. Ball & s.n. & 22 & 6 & 1862 \\
\hline$E$ & E00743410 & O. Debeaux & s.n. & 24 & 5 & 1867 \\
\hline$E$ & E00743409 & H. Hanry & s.n. & n.a. & 5 & 1867 \\
\hline$E$ & E00743407 & Elisée Reverchon & 340 & 14 & 6 & 1880 \\
\hline$E$ & E00743451 & E. Reverchon & s.n. & 17 & 6 & 1886 \\
\hline$E$ & E00743432 & E. Reverchon & s.n. & 19 & 6 & 1886 \\
\hline$E$ & E00743396 & Elisée Reverchon & s.n. & 19 & 6 & 1886 \\
\hline$E$ & E00743419 & G. Bentham & s.n. & n.a. & n.a. & ante $188<$ \\
\hline$E$ & E00743418 & W. Wilson Saund & $\in S . n$. & n.a. & n.a. & ante $188 \varepsilon$ \\
\hline $\mathrm{FI}$ & DSN112 & Durando (?) & s.n. & 14 & 6 & 1843 \\
\hline $\mathrm{FI}$ & DSN107 & Reuter & s.n. & n.a. & 8 & 1843 \\
\hline $\mathrm{FI}$ & DSN439 & P.F.A. Irat & s.n. & n.a. & 9 & 1845 \\
\hline $\mathrm{FI}$ & DSN417 & C. Marchesetti & 408 & 26 & 5 & 1849 \\
\hline $\mathrm{FI}$ & DSN384 & A. Chabers & s.n. & n.a. & 6 & 1851 \\
\hline $\mathrm{FI}$ & DSN444 & Ch. Pontarlier & s.n. & n.a. & $6--7$ & 1856 \\
\hline $\mathrm{FI}$ & DSN108 & Parlatere & s.n. & 22 & 6 & 1869 \\
\hline $\mathrm{FI}$ & DSN427 & Masson (?) & s.n. & 27 & 5 & 1870 \\
\hline $\mathrm{FI}$ & DSN431 & H. Hervier-Basso & s.n. & 27 & 5 & 1870 \\
\hline $\mathrm{FI}$ & DSN385 & A. Chabers & s.n. & 17 & 6 & 1881 \\
\hline $\mathrm{FI}$ & DSN443 & V. Bach & s.n. & n.a. & 7 & 1898 \\
\hline $\mathrm{FI}$ & DSN445 & Franqueville & s.n. & n.a. & 12 & 1841 \\
\hline $\mathrm{FI}$ & DSN446 & C. Flahault & s.n. & 14 & 6 & 1887 \\
\hline $\mathrm{FI}$ & DSN423 & G. Gavelle & s.n. & 5 & 6 & 1963 \\
\hline FR & FR-0121334 & J.-C. Ducommun & s.n. & 26 & 5 & 1859 \\
\hline FR & FR-0121331 & P.A. Kesselmeyer & s.n. & 20 & 6 & 1867 \\
\hline FR & FR-0121333 & N.Ch. Pontarlier & s.n. & 1 & 7 & 1880 \\
\hline FR & FR-0121345 & Stifani & s.n. & 4 & 7 & 1912 \\
\hline FR & FR-0121347 & R. Weber & s.n. & n.a. & 5 & 1966 \\
\hline G & P6094975 & Choisy & s.n. & n.a. & n.a. & 1824 \\
\hline G & P6095020 & Rodolph Rapin & s.n. & 5 & 6 & 1832 \\
\hline G & P6094885 & Requien & s.n. & n.a. & n.a. & 1836 \\
\hline G & P6014889 & Fauché & s.n. & n.a. & n.a. & 1840 \\
\hline G & P6094976 & A. de St Hilaire & s.n. & n.a. & 6 & 1846 \\
\hline G & P6094908 & Huet du Pavillon & s.n. & 22 & 5 & 1846 \\
\hline G & P6014884 & J. Lloyd & s.n. & 23 & 6 & 1851 \\
\hline G & P6014882 & Reuter & s.n. & n.a. & n.a. & 1852 \\
\hline G & P6094977 & Dr. Friedrich Sign & s.n. & 4 & 7 & 1857 \\
\hline G & P6095063 & Dr. Dupin & s.n. & n.a. & 6 & 1857 \\
\hline G & P6094904 & E. Ayasse & s.n. & 4 & 5 & 1859 \\
\hline G & P6094978 & Dr. Friedrich Sign & s.n. & 10 & 6 & 1860 \\
\hline G & P6094982 & Bélanger & s.n. & n.a. & n.a. & 1862 \\
\hline G & P6095076 & Fasson & s.n. & 26 & 4 & 1862 \\
\hline G & P6094909 & Alph. Moritz & s.n. & 10 & 5 & 1863 \\
\hline
\end{tabular}




\begin{tabular}{|c|c|c|c|c|c|c|}
\hline G & P6094905 & \multicolumn{2}{|c|}{ Ch. Verrier Livardis.n. } & 1 & 6 & 1873 \\
\hline G & P6094973 & J.L.A. Bonneau & s.n. & 2 & 7 & 1876 \\
\hline G & P6094887 & Dr. A. Bétrix & s.n. & 10 & 6 & 1877 \\
\hline G & P6094985 & Bélanger & s.n. & n.a. & 5 & 1890 \\
\hline G & P6095059 & Louis Amblard & s.n. & 20 & 8 & 1890 \\
\hline G & P6095070 & \multicolumn{2}{|c|}{ Baraize, A. Perrier s.n. } & n.a. & 6 & 1892 \\
\hline G & P6095069 & Roux, A. Perrier & s.n. & n.a. & n.a. & 1893 \\
\hline G & P6094888 & W. Gugelmann & $37 / 224$ & 18 & 6 & 1907 \\
\hline G & P6094911 & \multicolumn{2}{|c|}{ Louis-André Giroc s.n. } & n.a. & 6 & 1910 \\
\hline G & P6094907 & M.K. Faust & s.n. & 26 & 5 & 1938 \\
\hline G & P6094996 & Simone Vautier & s.n. & 13 & 6 & 1948 \\
\hline G & P6094928 & Duperrex-Weber & s.n. & 14 & 6 & 1948 \\
\hline G & P6094927 & Duperrex-Weber & s.n. & 8 & 6 & 1948 \\
\hline G & P6094995 & L. Vautier & s.n. & 24 & 5 & 1949 \\
\hline G & P6094980 & Charles Bélanger & s.n. & n.a. & n.a. & ante $186<$ \\
\hline G & P6094983 & Dr. Montagne & s.n. & n.a. & n.a. & ante $186 t$ \\
\hline G & P6094972 & Chambion (?) & s.n. & n.a. & 6 & ante $187 \subseteq$ \\
\hline G & P6094971 & Cadet & s.n. & n.a. & 6 & ante $187 \subseteq$ \\
\hline G & P6095066 & Delessert & s.n. & n.a. & n.a. & ante $189 t$ \\
\hline G & P6095066 & Delessert & s.n. & n.a. & n.a. & ante $189 €$ \\
\hline G & P6094979 & Garrot & s.n. & n.a. & n.a. & ante $190 \equiv$ \\
\hline G & P6094886 & \multicolumn{2}{|c|}{ Abbé Roquebrunєs.n. } & n.a. & 7 & ante 190; \\
\hline HUJ & HUJHERB 131644 & A. Danin & $72-14-23$ & 13 & 6 & 1972 \\
\hline K & K001092999 & leg. ign. & 7 & 18 & 5 & 1825 \\
\hline K & K001042848 & E. Serafino & 1487.1 & n.a. & 3 & 1828 \\
\hline K & K001042835 & Liendon & s.n. & n.a. & 6 & 1847 \\
\hline K & K001042840 & E. Bourgeau & 2 & 25 & 5 & 1848 \\
\hline K & K001042801 & leg. ign. & s.n. & 3 & 4 & 1867 \\
\hline K & K001042805 & J.M.H. Borel & s.n. & 10 & 6 & 1867 \\
\hline K & K001042800 & leg. ign. & s.n. & 14 & 5 & 1869 \\
\hline K & K001042815 & J.C. Joad & s.n. & 24 & 4 & 1870 \\
\hline K & K001092994 & Ed. Bonnet & s.n. & 4 & 9 & 1875 \\
\hline K & K001092996 & A. Cartier & s.n. & 20 & 5 & 1877 \\
\hline K & K001092995 & Ed. Marçais & s.n. & n.a. & $6--7$ & 1878 \\
\hline K & K001092997 & P. & s.n. & n.a. & 7 & 1899 \\
\hline K & K001042806 & \multicolumn{2}{|c|}{ E. Ellman \& N.Y. S 99} & 6 & 6 & 1925 \\
\hline K & K001042804 & F.N. Hepper & 8945 & 26 & 5 & 1990 \\
\hline K & K001042918 & Requien & s.n. & n.a. & n.a. & ante $186^{-}$ \\
\hline K & K001264135 & M. Requien & s.n. & n.a. & n.a. & ante 186; \\
\hline K & K001042807 & Lombardelly & s.n. & n.a. & 6 & ante 1921 \\
\hline MA & MA-01-00156308 & Mutel & s.n. & n.a. & 5 & 1843 \\
\hline MA & MA-01-00038767 & F. Bruyas & 2629 & n.a. & 6 & 1891 \\
\hline MA & MA-01-00038765 & Fre. Sennen & s.n. & 6 & 7 & 1891 \\
\hline MA & MA-01-00650348 & J.F. de Witte & 15027 & 19 & 5 & 1961 \\
\hline
\end{tabular}




\begin{tabular}{|c|c|c|c|c|c|c|}
\hline MA & MA-01-00191210 & G. Gavelle & s.n. & 5 & 6 & 1963 \\
\hline MA & MA-01-00290893 & G. Gavelle & s.n. & 11 & 6 & 1965 \\
\hline MA & MA-01-00290896 & G. Gavelle & s.n. & 15 & 7 & 1965 \\
\hline MA & MA-01-00650332 & J.F. de Witte & 15814 & 20 & 5 & 1965 \\
\hline MA & MA-01-00650333 & J.F. de Witte & 16909 & 13 & 6 & 1967 \\
\hline MA & MA-01-00650349 & J.F. de Witte & 16269 & 2 & 6 & 1967 \\
\hline $\mathrm{MA}$ & MA-01-00204222 & Bellot, Casaseca & s.n. & 25 & 5 & 1972 \\
\hline MEL & MEL-2407544 & Viguier & s.n. & n.a. & 7 & 1839 \\
\hline MEL & MEL-2319016 & John Stuart Mill & s.n. & n.a. & 5 & 1859 \\
\hline MPU & n.a. & Herbier fac de mé & s.n. & n.a. & n.a. & 1803 \\
\hline MPU & n.a. & Guillon & s.n. & 16 & 6 & 1820 \\
\hline MPU & n.a. & Herbier fac de mé & s.n. & 2 & 6 & 1828 \\
\hline MPU & n.a. & Dunal & s.n. & 31 & 5 & 1834 \\
\hline MPU & n.a. & Cambessèdes & s.n. & 17 & 6 & 1835 \\
\hline MPU & n.a. & F. de Girard & s.n. & 25 & 6 & 1837 \\
\hline MPU & n.a. & A. Irat & s.n. & n.a. & 5 & 1842 \\
\hline MPU & n.a. & E. Cosson & s.n. & 4 & 6 & 1846 \\
\hline MPU & n.a. & L. Liendon & 391 a & n.a. & 6 & 1847 \\
\hline MPU & n.a. & Planchon & s.n. & n.a. & 5 & 1847 \\
\hline MPU & n.a. & Liendon & 391 & n.a. & 6 & 1847 \\
\hline MPU & n.a. & Guillon & s.n. & 2 & 7 & 1849 \\
\hline MPU & n.a. & P.F.A. Irat & s.n. & n.a. & 9 & 1849 \\
\hline MPU & n.a. & P.F.A. Irat & s.n. & 26 & 5 & 1849 \\
\hline MPU & n.a. & E. Le Sourd-Dussis & s.n. & n.a. & 5 & 1849 \\
\hline MPU & n.a. & Hubert & s.n. & 21 & 6 & 1851 \\
\hline MPU & n.a. & Dr Le Sourd & s.n. & 26 & 6 & 1852 \\
\hline MPU & n.a. & Planchon & s.n. & n.a. & 6 & 1854 \\
\hline MPU & n.a. & L. Pierre & s.n. & n.a. & n.a. & 1858 \\
\hline MPU & n.a. & H. Hanry & s.n. & 6 & 5 & 1860 \\
\hline MPU & n.a. & H. Hanry & 3003 & 6 & 5 & 1860 \\
\hline MPU & n.a. & Dr Le Sourd & s.n. & 29 & 5 & 1862 \\
\hline MPU & n.a. & Aubouy & s.n. & 3 & 6 & 1863 \\
\hline MPU & n.a. & Aubouy & s.n. & 3 & 6 & 1863 \\
\hline MPU & n.a. & leg. ign. & s.n. & 20 & 5 & 1864 \\
\hline MPU & n.a. & Herbier André & s.n. & n.a. & n.a. & 1864 \\
\hline MPU & n.a. & Dr Le Sourd & s.n. & n.a. & 5 & 1866 \\
\hline MPU & n.a. & Aubouy & s.n. & 18 & 5 & 1867 \\
\hline MPU & n.a. & Aubouy & s.n. & 29 & 5 & 1870 \\
\hline MPU & n.a. & Brunet & s.n. & 20 & 5 & 1871 \\
\hline MPU & n.a. & Bernardin Martin & s.n. & n.a. & 6 & 1871 \\
\hline MPU & n.a. & A. Tholin & s.n. & n.a. & 4 & 1876 \\
\hline MPU & n.a. & Neyra & s.n. & 23 & 5 & 1876 \\
\hline MPU & n.a. & A.B. & s.n. & 22 & 6 & 1876 \\
\hline MPU & n.a. & Aubouy & s.n. & 4 & 7 & 1876 \\
\hline
\end{tabular}




\begin{tabular}{|c|c|c|c|c|c|c|}
\hline MPU & n.a. & A. Cartier & s.n. & 20 & 5 & 1877 \\
\hline MPU & n.a. & A. Cartier & s.n. & 20 & 5 & 1877 \\
\hline MPU & n.a. & J. Daveau & s.n. & n.a. & 5 & 1879 \\
\hline MPU & n.a. & André & s.n. & n.a. & n.a. & 1882 \\
\hline MPU & n.a. & Aubouy & s.n. & 30 & 6 & 1884 \\
\hline MPU & n.a. & E.J. Neyraut & s.n. & 29 & 5 & 1886 \\
\hline MPU & n.a. & \multicolumn{2}{|c|}{ docteur E. Magnos.n. } & n.a. & 6 & 1886 \\
\hline MPU & n.a. & F. Mouret & s.n. & n.a. & 6 & 1886 \\
\hline MPU & n.a. & E.J. Neyraut & 4794 & 3 & 6 & 1887 \\
\hline MPU & n.a. & E.J. Neyraut & 4794 & 3 & 6 & 1887 \\
\hline MPU & n.a. & E. Magnol & s.n. & 22 & 6 & 1887 \\
\hline MPU & n.a. & \multicolumn{2}{|c|}{ Herbier C. Flahauts.n. } & 14 & 6 & 1887 \\
\hline MPU & n.a. & Aubouy & s.n. & 31 & 5 & 1889 \\
\hline MPU & n.a. & Abbe A. Pons & s.n. & 12 & 6 & 1889 \\
\hline MPU & n.a. & F. Bruyas & s.n. & n.a. & 6 & 1891 \\
\hline MPU & n.a. & A. Guillon & s.n. & 7 & 7 & 1892 \\
\hline MPU & n.a. & E.J. Neyraut & s.n. & 28 & 5 & 1893 \\
\hline MPU & n.a. & C. Copineau & s.n. & 26 & 5 & 1893 \\
\hline MPU & n.a. & Bertrand & 217 & 10 & 6 & 1894 \\
\hline MPU & n.a. & Dr. Jouvence (?) & s.n. & n.a. & 5 & 1896 \\
\hline MPU & n.a. & A. Raphélis & s.n. & n.a. & 5 & 1896 \\
\hline MPU & n.a. & Faure & 120 & 3 & 6 & 1898 \\
\hline MPU & n.a. & Faure & s.n. & 3 & 6 & 1898 \\
\hline MPU & n.a. & Frère Sennen & s.n. & n.a. & n.a. & 1899 \\
\hline MPU & n.a. & Frère Sennen & s.n. & n.a. & n.a. & 1899 \\
\hline MPU & n.a. & Cassat & s.n. & 5 & 7 & 1899 \\
\hline MPU & n.a. & Frère Sennen & s.n. & n.a. & n.a. & 1899 \\
\hline MPU & n.a. & C.A. Gérard & 4674 & 12 & 6 & 1901 \\
\hline MPU & n.a. & Frère Sennen & s.n. & n.a. & 5 & 1901 \\
\hline MPU & n.a. & A. Guillon & s.n. & 30 & 6 & 1902 \\
\hline MPU & n.a. & J. Stefani & s.n. & 10 & 6 & 1903 \\
\hline MPU & n.a. & Fourès & s.n. & n.a. & 6 & 1907 \\
\hline MPU & n.a. & J. de Vichet & s.n. & 12 & 6 & 1914 \\
\hline MPU & n.a. & J. de Vichet & s.n. & 28 & 5 & 1914 \\
\hline MPU & n.a. & P. Cousturier & s.n. & n.a. & 4 & 1918 \\
\hline MPU & n.a. & Albaille & s.n. & 15 & 4 & 1918 \\
\hline MPU & n.a. & Albaille & s.n. & 15 & 4 & 1918 \\
\hline MPU & n.a. & J.B. Renaud & s.n. & 5 & 6 & 1926 \\
\hline MPU & n.a. & E.J. Neyraut & s.n. & 14 & 10 & 1936 \\
\hline MPU & n.a. & leg. ign. & s.n. & 12 & 6 & 1956 \\
\hline MPU & n.a. & S.J. Albaille & s.n. & 13 & 6 & 1959 \\
\hline MPU & n.a. & S.J. Albaille & s.n. & 10--20 & 6 & 1959 \\
\hline MPU & n.a. & G. Gavelle & 4269 & 15 & 6 & 1963 \\
\hline MPU & n.a. & G. Blanchet & s.n. & n.a. & 6 & 1965 \\
\hline
\end{tabular}




\begin{tabular}{|c|c|c|c|c|c|c|}
\hline MPU & n.a. & G. Blanchet & s.n. & 12 & 6 & 1966 \\
\hline MPU & n.a. & Santo & s.n. & 12 & 6 & 1966 \\
\hline MPU & n.a. & E.J. Neyraut & s.n. & 29--1 & $5--7$ & $1893 \& 1 \imath$ \\
\hline$P$ & P03175036 & leg. ign. & 117 & 22 & 5 & 1817 \\
\hline$P$ & P03174919 & Jacquemont & s.n. & n.a. & 5 & 1821 \\
\hline$P$ & P03175003 & Leman & s.n. & n.a. & n.a. & 1821 \\
\hline$P$ & P03174952 & A.P. de Candolle & s.n. & n.a. & n.a. & 1822 \\
\hline$P$ & P02810831 & M. Emerie (?) & s.n. & n.a. & n.a. & 1827 \\
\hline$P$ & P03175014 & W. de Schoenefe & s.n. & n.a. & n.a. & 1828 \\
\hline$P$ & P03174939 & M. Bentham & s.n. & n.a. & n.a. & 1834 \\
\hline$P$ & P02391570 & $\mathrm{R}$ & s.n. & n.a. & 6 & 1834 \\
\hline$P$ & P02391570 & $\mathrm{R}$. & s.n. & n.a. & 6 & 1834 \\
\hline$P$ & P02575181 & Dunal & s.n. & n.a. & n.a. & 1835 \\
\hline$P$ & P03174925 & Perreymond & s.n. & n.a. & n.a. & 1836 \\
\hline$P$ & P03174925 & Perreymond & s.n. & n.a. & n.a. & 1836 \\
\hline$P$ & P03174938 & A. Lenormand & s.n. & n.a. & n.a. & 1838 \\
\hline$P$ & P02391512 & P.F.A. Irat & 753 & 15 & 6 & 1842 \\
\hline$P$ & P03175006 & Callou (?) & s.n. & n.a. & 7 & 1844 \\
\hline$P$ & P03174974 & Schler (?) & s.n. & n.a. & 5 & 1845 \\
\hline$P$ & P02391534 & A. Hertz (?) & s.n. & 5 & 8 & 1845 \\
\hline$P$ & P03174947 & A. de St Hilaire & s.n. & n.a. & 6 & 1845 \\
\hline$P$ & P02575074 & Cavalier & s.n. & n.a. & n.a. & 1845 \\
\hline$P$ & P02575099 & A. de St Hilaire & s.n. & n.a. & 6 & 1845 \\
\hline$P$ & P02575069 & Fée & s.n. & n.a. & n.a. & 1845 \\
\hline$P$ & P03175022 & Henrici Lartigue & s.n. & 15 & 7 & 1845 \\
\hline$P$ & P02575075 & F. Clement & s.n. & n.a. & n.a. & 1846 \\
\hline$P$ & P02575186 & A.G. de Dives & 18 & 10 & 6 & 1846 \\
\hline$P$ & P03174965 & De Lort & s.n. & n.a. & n.a. & 1846 \\
\hline$P$ & P03174951 & Dr. Grenier & s.n. & n.a. & 7 & 1846 \\
\hline$P$ & P03174979 & A. Leclerc & s.n. & n.a. & 8 & 1847 \\
\hline$P$ & P02575080 & Boyé & 50 & n.a. & n.a. & 1847 \\
\hline$P$ & P02575073 & L. Kralik & 1849 & 4 & 6 & 1847 \\
\hline$P$ & P03175011 & Liendon & s.n. & n.a. & n.a. & 1847 \\
\hline$P$ & P03174935 & Liendon & s.n. & n.a. & 6 & 1847 \\
\hline$P$ & P03175018 & A. Guillon & s.n. & 11 & 6 & 1850 \\
\hline$P$ & P03175029 & Leclerc & s.n. & n.a. & n.a. & 1850 \\
\hline$P$ & P02530409 & Orth. André & s.n. & n.a. & 6 & 1850 \\
\hline$P$ & P02575180 & J. Lloyd & s.n. & n.a. & 6 & 1851 \\
\hline$P$ & P03174969 & Ch. Gontartiez & s.n. & n.a. & $7--8$ & 1852 \\
\hline$P$ & P02575209 & Ch. Pontarlier & s.n. & n.a. & 6 & 1852 \\
\hline$P$ & P03174964 & Louis Amblard & s.n. & 21 & 6 & 1853 \\
\hline$P$ & P02575065 & Loret & s.n. & n.a. & n.a. & 1854 \\
\hline$P$ & P02575208 & \multicolumn{2}{|c|}{ Institut Botaniques.n. } & n.a. & 9 & 1854 \\
\hline$P$ & P02575066 & leg. ign. & s.n. & n.a. & 8--9 & 1854 \\
\hline
\end{tabular}




\begin{tabular}{|c|c|c|c|c|c|c|}
\hline$P$ & P03174968 & Lepeltier & s.n. & n.a. & 7 & 1856 \\
\hline$P$ & P03174994 & J. Parseval & s.n. & 7 & 6 & 1859 \\
\hline$P$ & P02569726 & Laguesse A. & s.n. & 17 & 6 & 1859 \\
\hline$P$ & P03174928 & C. Billot & \multicolumn{2}{|c|}{3003 (1868 n n.a. } & $5--6$ & 1860 \\
\hline$P$ & P03174977 & Ch. Ozanon & s.n. & n.a. & 6 & 1860 \\
\hline $\mathrm{P}$ & P03174963 & Ch. Ozanon & s.n. & n.a. & 6 & 1860 \\
\hline$P$ & P03174926 & G. Thuret & 61 & 20 & 6 & 1860 \\
\hline$P$ & P03175034 & \multicolumn{2}{|c|}{ H. de la Perraudiè s.n. } & 27 & 6 & 1860 \\
\hline$P$ & P02530405 & L'Abbé Lelièvre & s.n. & 3 & 6 & 1860 \\
\hline$P$ & P02575210 & D.M. Roux & s.n. & n.a. & 6 & 1860 \\
\hline$P$ & P02575210 & D.M. Roux & s.n. & n.a. & 6 & 1860 \\
\hline$P$ & P03175002 & [illegible] & s.n. & n.a. & 4 & 1861 \\
\hline$P$ & P02570013 & E. Bourgeau & s.n. & 17 & 5 & 1861 \\
\hline$P$ & P03175024 & E. Bourgeau & s.n. & 27 & 5 & 1861 \\
\hline $\mathrm{P}$ & P03175030 & E. Bourgeau & s.n. & 27 & 5 & 1861 \\
\hline$P$ & P03174944 & E. Bourgeau & s.n. & 27 & 5 & 1861 \\
\hline$P$ & P03174994 & E. Bourgeau & s.n. & 27 & 5 & 1861 \\
\hline$P$ & P03174953 & Dr Lebel & s.n. & n.a. & 6 & 1862 \\
\hline $\mathrm{P}$ & P00685973 & A. Legrand & s.n. & 1 & 7 & 1862 \\
\hline$P$ & P03174962 & Dr Bras & s.n. & 21 & 7 & 1862 \\
\hline$P$ & P03175017 & Bocquillon & s.n. & n.a. & 6 & 1863 \\
\hline$P$ & P02569725 & Th. Delacour & s.n. & n.a. & 6 & 1863 \\
\hline$P$ & P02575067 & E.M. Sauvage & s.n. & 27 & 5 & 1863 \\
\hline $\mathrm{P}$ & P03174923 & Marcilly & s.n. & 29 & 5 & 1865 \\
\hline$P$ & P03174927 & Bureau & s.n. & 22 & 5 & 1865 \\
\hline$P$ & P03175001 & A. Franchet & s.n. & n.a. & 6 & 1866 \\
\hline$P$ & P02569729 & Th. Delacour & s.n. & 26 & 5 & 1866 \\
\hline$P$ & P02569657 & C. Damiens & s.n. & $4--5$ & 6 & 1867 \\
\hline $\mathrm{P}$ & P02391605 & H. van Heurck & s.n. & n.a. & n.a. & 1867 \\
\hline$P$ & P03174997 & O. Debeaux & s.n. & 6 & 6 & 1869 \\
\hline$P$ & P02305581 & Warnier & 22 & n.a. & n.a. & 1869 \\
\hline$P$ & P02575211 & Chauvin & s.n. & n.a. & 6 & 1870 \\
\hline$P$ & P02569998 & \multicolumn{2}{|c|}{ H. Hervier-Basson s.n. } & 27 & 5 & 1870 \\
\hline$P$ & P02570065 & leg. ign. & s.n. & 29 & 5 & 1870 \\
\hline$P$ & P02570009 & Th. Delacour & s.n. & 27 & 5 & 1871 \\
\hline$P$ & P02570001 & Ch. Pontarlier & s.n. & 4 & 7 & 1871 \\
\hline$P$ & P03175033 & M. Franchet & s.n. & n.a. & n.a. & 1872 \\
\hline$P$ & P02569656 & Th. Delacour & s.n. & $1--2$ & 7 & 1873 \\
\hline$P$ & P05591715 & leg. ign. & s.n. & n.a. & n.a. & 1873 \\
\hline$P$ & P02569993 & $\mathrm{H}($ ?)aures & s.n. & n.a. & 5 & 1874 \\
\hline$P$ & P02569979 & L. Anthouard & s.n. & 1 & 6 & 1875 \\
\hline $\mathrm{P}$ & P03174940 & leg. ign. & s.n. & n.a. & 5 & 1875 \\
\hline$P$ & P02530406 & R.B. & s.n. & 4 & 7 & 1875 \\
\hline$P$ & P03174993 & A. Cartier & s.n. & n.a. & 5 & 1875 \\
\hline
\end{tabular}




\begin{tabular}{|c|c|c|c|c|c|c|}
\hline$P$ & P03174993 & A. Cartier & s.n. & n.a. & 5 & 1875 \\
\hline$P$ & P02570004 & \multicolumn{2}{|c|}{ J. Gautier-Lacroze s.n. } & n.a. & 6 & 1875 \\
\hline$P$ & P03174990 & E. Reverchon & s.n. & 27 & 6 & 1877 \\
\hline$P$ & P03174987 & Ed. Marçais & s.n. & n.a. & 8 & 1877 \\
\hline$P$ & P02575169 & J. Daveau & s.n. & 28 & 5 & 1878 \\
\hline$P$ & P02575207 & E. Marçay & s.n. & n.a. & $6--7$ & 1878 \\
\hline$P$ & P02569994 & E. Marçay & s.n. & n.a. & $6--7$ & 1878 \\
\hline$P$ & P02570005 & E. Marçay & s.n. & n.a. & $6--7$ & 1878 \\
\hline$P$ & P02391524 & leg. ign. & s.n. & 20 & 7 & 1878 \\
\hline$P$ & P02391524 & leg. ign. & s.n. & 20 & 7 & 1878 \\
\hline$P$ & P03174988 & \multicolumn{2}{|c|}{ V. Retalon (or Me s.n. } & 20 & 8 & 1878 \\
\hline$P$ & P02570113 & J.B. Barla & s.n. & n.a. & 6 & 1881 \\
\hline$P$ & P02569655 & Th. Delacour & s.n. & 10 & 6 & 1886 \\
\hline$P$ & P03174934 & E. Reverchon & s.n. & 14 & 6 & 1886 \\
\hline$P$ & P03174992 & E. Reverchon & s.n. & 19 & 6 & 1886 \\
\hline$P$ & P02570104 & leg. ign. & s.n. & 6 & 7 & 1886 \\
\hline$P$ & P03174986 & L. Chevallier & s.n. & 22 & 6 & 1887 \\
\hline$P$ & P03175021 & V. Auzat & s.n. & 28 & 7 & 1888 \\
\hline$P$ & P02391521 & Hubert & s.n. & 21 & 6 & 1891 \\
\hline$P$ & P02575185 & J.B. Barla & s.n. & 30 & 5 & 1892 \\
\hline$P$ & P02575204 & E. Dupuy & s.n. & n.a. & 6 & 1894 \\
\hline$P$ & P02575184 & J.B. Barla & s.n. & 23 & 5 & 1895 \\
\hline$P$ & P02575096 & Ch. Hermant & s.n. & n.a. & $6--7$ & 1897 \\
\hline$P$ & P02575206 & S. Savouré & $562 c$ & 7 & 6 & 1897 \\
\hline$P$ & P02575219 & J. Bozon & s.n. & 12 & 6 & 1898 \\
\hline$P$ & P03174980 & R. Rotgès & s.n. & 28 & 5 & 1898 \\
\hline$P$ & P02575183 & Cassat & s.n. & 5 & 7 & 1899 \\
\hline$P$ & P02570098 & Fre Sennen & s.n. & n.a. & n.a. & 1899 \\
\hline $\mathrm{P}$ & P03175040 & H. Coste & s.n. & 13 & 7 & 1899 \\
\hline$P$ & P02575205 & S. Savouré & 695 & 16 & 11 & 1899 \\
\hline$P$ & P02570111 & H. Coste & 323 & n.a. & 7 & 1900 \\
\hline$P$ & P02570111 & H. Coste & 323 & n.a. & 7 & 1900 \\
\hline$P$ & P02575188 & J. Strici & s.n. & 29 & 4 & 1901 \\
\hline$P$ & P02575187 & Ciprillon & s.n. & 30 & 6 & 1902 \\
\hline$P$ & P03175025 & Hoschedé & s.n. & 17 & 6 & 1902 \\
\hline$P$ & P02569730 & Sennen & s.n. & n.a. & 5 & 1902 \\
\hline$P$ & P02575189 & E. Coraze & s.n. & 25 & 6 & 1902 \\
\hline$P$ & P00620192 & Legris & s.n. & 3 & 7 & 1902 \\
\hline$P$ & P04560803 & Legris & s.n. & 3 & 7 & 1902 \\
\hline$P$ & P02523825 & Ch. Guffroy & 111 & n.a. & n.a. & 1903 \\
\hline$P$ & P03174950 & G. Hibon & 111 & n.a. & n.a. & 1903 \\
\hline $\mathrm{P}$ & P03174950 & G. Hibon & 111 & n.a. & n.a. & 1903 \\
\hline$P$ & P03174942 & G. Hibon & 111 & n.a. & 6 & 1905 \\
\hline$P$ & P02575157 & W.R. Fontan & s.n. & 15 & 6 & 1905 \\
\hline
\end{tabular}




\begin{tabular}{|c|c|c|c|c|c|c|}
\hline$P$ & P02570110 & L. Bee & 321 & n.a. & 6 & 1906 \\
\hline$P$ & P03175013 & Dr. Lermoyez & 93 & n.a. & n.a. & 1906 \\
\hline$P$ & P02575137 & J. de Vichet & s.n. & n.a. & 6 & 1907 \\
\hline$P$ & P03174982 & A. et E.G. Camus & s.n. & 19 & 6 & 1909 \\
\hline$P$ & P02575098 & Ch. Guffroy & s.n. & 19 & 6 & 1910 \\
\hline$P$ & P03174948 & Humbert & s.n. & 6 & 6 & 1910 \\
\hline$P$ & P03174984 & E.G. Camus & s.n. & n.a. & 6 & 1911 \\
\hline $\mathrm{P}$ & P03174966 & L'abbé Somers & s.n. & 15 & 1 & 1911 \\
\hline$P$ & P02575164 & J.J. Weber & s.n. & 29 & 5 & 1912 \\
\hline $\mathrm{P}$ & P02575077 & J.B. Renaud & s.n. & n.a. & $5--6$ & 1914 \\
\hline $\mathrm{P}$ & P02575101 & J.B. Renaud & s.n. & n.a. & $5--6$ & 1914 \\
\hline$P$ & P02559213 & Jean Guillet & 93 & 29 & 7 & 1914 \\
\hline $\mathrm{P}$ & P03175016 & L. Rodriguez & s.n. & 7 & 9 & 1917 \\
\hline $\mathrm{P}$ & P02570112 & J. Arènes & 324 & n.a. & 5 & 1919 \\
\hline$P$ & P02570066 & leg. ign. & s.n. & 6 & 5 & 1923 \\
\hline$P$ & P03174958 & \multicolumn{2}{|c|}{ Mariane de Gougıs.n. } & n.a. & n.a. & 1925 \\
\hline$P$ & P02575166 & J. Arbost & s.n. & 15 & 6 & 1926 \\
\hline$P$ & P03174956 & \multicolumn{2}{|c|}{ Mariane de Gougıs.n. } & n.a. & 7 & 1927 \\
\hline$P$ & P02575144 & B. de Retz & 284 & 21 & 7 & 1928 \\
\hline$P$ & P03174955 & \multicolumn{2}{|c|}{ Mariane de Gougıs.n. } & $\mathrm{v}$ & 6 & 1930 \\
\hline $\mathrm{P}$ & P02575139 & L. Samat & 273 bis & 25 & 5 & 1930 \\
\hline $\mathrm{P}$ & P02570109 & L. Samat & 322 & 25 & 5 & 1930 \\
\hline$P$ & P02559215 & CL. Legros & s.n. & 5 & 6 & 1930 \\
\hline$P$ & P02575136 & B. de Retz & 273 ter & 14 & 7 & 1932 \\
\hline$P$ & P03187614 & E. Delpature & s.n. & n.a. & 6 & 1932 \\
\hline $\mathrm{P}$ & P02575178 & R. L'Hermite & s.n. & 3 & 6 & 1936 \\
\hline $\mathrm{P}$ & P04560804 & L. Rotereau & s.n. & n.a. & 6 & 1936 \\
\hline $\mathrm{P}$ & P02575142 & B. de Retz & 14753 & 7 & 6 & 1938 \\
\hline$P$ & P02575163 & Mme Haffner & s.n. & 14 & 8 & 1938 \\
\hline$P$ & P02575072 & Bournerias & 1534 & n.a. & 6 & 1940 \\
\hline $\mathrm{P}$ & P00731924 & E. Licent & s.n. & 3 & 6 & 1941 \\
\hline $\mathrm{P}$ & P02559210 & Henri Bouby & 860 & n.a. & 8 & 1942 \\
\hline $\mathrm{P}$ & P03187615 & E. Delpature & s.n. & n.a. & 6 & 1943 \\
\hline $\mathrm{P}$ & P02575143 & B. de Retz & 23558 & 2 & 6 & 1947 \\
\hline $\mathrm{P}$ & P02559212 & Henri Bouby & 10.017 & 6 & 6 & 1948 \\
\hline $\mathrm{P}$ & P02569701 & H. Gillet & s.n. & 15 & 5 & 1954 \\
\hline $\mathrm{P}$ & P02575159 & B. de Retz & 39707 & 10 & 6 & 1956 \\
\hline $\mathrm{P}$ & P02575158 & B. de Retz & 39603 & 9 & 6 & 1956 \\
\hline $\mathrm{P}$ & P02559214 & J. Charrier & s.n. & 19 & 6 & 1957 \\
\hline $\mathrm{P}$ & P02575097 & J. Charrier & 3363 & 30 & 6 & 1958 \\
\hline $\mathrm{P}$ & P02559216 & J. Raynal & 2966 & 13 & 6 & 1958 \\
\hline $\mathrm{P}$ & P03024565 & \multicolumn{2}{|c|}{ Jacqueline Tatignıs.n. } & 22 & 6 & 1959 \\
\hline $\mathrm{P}$ & P02575070 & E. Contré & 4268 & 22 & 6 & 1962 \\
\hline $\mathrm{P}$ & P02589843 & Paul Jovet & s.n. & 13 & 7 & 1969 \\
\hline
\end{tabular}




\begin{tabular}{|c|c|c|c|c|c|c|}
\hline $\mathrm{P}$ & P03618090 & \multicolumn{2}{|c|}{ G.G. Aymonin et $\uparrow 28281$} & 6 & 6 & 1973 \\
\hline$P$ & P03618089 & \multicolumn{2}{|c|}{ G.G. Aymonin et 128271} & 6 & 6 & 1973 \\
\hline$P$ & P03174972 & Requien & s.n. & n.a. & n.a. & ante $186 \varepsilon$ \\
\hline PAL & PAL-Gr 100355 & Dr. Antoine Bras & s.n. & 21 & 7 & 1861 \\
\hline S & S16-2583 & Reverchon & s.n. & 14 & 6 & 1880 \\
\hline$S$ & S16-2850 & Ch. Pont $[\ldots]$ & s.n. & 1 & 7 & 1883 \\
\hline S & S16-2827 & Carl de Geer & s.n. & 8 & 4 & 1883 \\
\hline$S$ & S16-2603 & Gombault & s.n. & 7 & 6 & 1903 \\
\hline S & S16-2584 & C. Bicknell & s.n. & 4 & 5 & 1906 \\
\hline S & S14-14265 & \multicolumn{2}{|c|}{ Hj. \& A. Hafstroens.n. } & 18 & 7 & 1911 \\
\hline S & S16-2912 & Hj. Hafstroem & s.n. & 18 & 7 & 1911 \\
\hline TO & dscn5682 & [illegible] & s.n. & 17 & 6 & 1873 \\
\hline TO & dscn5647 & Baraize & s.n. & n.a. & 6 & 1882 \\
\hline TO & dscn5660 & J. Stefani & s.n. & 10 & 6 & 1903 \\
\hline UPS & V-738373 & \multicolumn{2}{|c|}{ Meddelad af S.N. S.n. } & n.a. & n.a. & 1823 \\
\hline UPS & V-738026 & \multicolumn{2}{|c|}{ A. de Saint-Hilaire s.n. } & n.a. & 5 & 1846 \\
\hline UPS & V-738322 & Smet[...] (?) & s.n. & n.a. & 5 & 1855 \\
\hline UPS & V-738025 & \multicolumn{2}{|c|}{ Alphonse Authems.n. } & n.a. & 6 & 1872 \\
\hline UPS & V-738025 & \multicolumn{2}{|c|}{ Alphonse Authems.n. } & n.a. & 6 & 1872 \\
\hline UPS & V-737956 & Ch. Pontarlier & s.n. & 10 & 7 & 1880 \\
\hline UPS & V-738372 & Carl de Geer & s.n. & n.a. & 5 & 1883 \\
\hline UPS & V-738355 & Elisée Reverchon & s.n. & 20 & 5 & 1886 \\
\hline UPS & V-738006 & Carl de Geer & s.n. & 8 & 4 & 1893 \\
\hline W & $1996-11639$ & A. Tillet & s.n. & n.a. & 7 & 1874 \\
\hline W & $1996-11638$ & Cartier & s.n. & n.a. & 5 & 1895 \\
\hline W & 16409 & Marnac & 6 & 19 & 6 & 1911 \\
\hline W & 2000-13105 & Froebe & 7832 & 21 & 5 & 1977 \\
\hline
\end{tabular}




\begin{tabular}{|c|c|c|c|c|}
\hline Country & Collection locality & \multicolumn{3}{|c|}{ Other notes c Flower morpl Presence of c } \\
\hline France & Champs et vignes prés Auch (Ger.) & n.a. & $\mathrm{P}$ & Yes \\
\hline France & Coteaux du Vinaigrié près Nice. & Relighiae Mai & $\mathrm{P}$ & Yes \\
\hline France & champs Montpellier & Relighiae Mai & $\mathrm{P}$ & No \\
\hline France & Strassburg, Neudorf alter (...?) & plena & $\mathrm{T}$ & Yes \\
\hline France & cambes (gir) & Dans les haie: & $\mathrm{T}$ & Yes \\
\hline France & aude: la nouvelle & Friche & $\mathrm{P}$ & Yes \\
\hline France & Corse: Bonifacio & sous les olivi $\epsilon$ & $P$ & Yes \\
\hline France & Bouches-du-Rhone: luynes & champs et lie & $P$ & Yes \\
\hline France & \multicolumn{2}{|c|}{ Bouches-du-Rhone: La Valentine. Friche in.a. } & $P$ & Yes \\
\hline France & Grasse & Talus, fossés & $P$ & No \\
\hline France & Lyon & n.a. & $\mathrm{P}$ & No \\
\hline France & Saintes, Charente Inf. & n.a. & $P$ & Yes \\
\hline France & Env. de Toulouse & n.a. & $\mathrm{P}$ & No \\
\hline France & Rogliano & n.a. & $P$ & No \\
\hline France & Mt. Pharon, près Toulon & n.a. & $P$ & No \\
\hline France & Roquefavour & n.a. & $\mathrm{P}$ & Yes \\
\hline France & St. Canat (Bouches-du- Rhône) & n.a. & $P$ & Yes \\
\hline France & St. Canat (Bouches-du- Rhône) & n.a. & $\mathrm{T}$ & Yes \\
\hline France & \multicolumn{2}{|c|}{ Moissons a Soubise (Charente-Inférieure n.a. } & $\mathrm{P}$ & Yes \\
\hline France & Bastia, Insel Corsika & Früchte & $\mathrm{P}$ & Yes \\
\hline France & Bastia & Collium incult & $\mathrm{P}$ & No \\
\hline France & Bastia & Collium incult & $\mathrm{T}$ & No \\
\hline France & \multicolumn{2}{|c|}{ Environs de Costebelle, au dessus du Car Terrain calcai } & $P$ & Yes \\
\hline France & [illegible] près de Gorniès & Moissons de & $\mathrm{P}$ & Yes \\
\hline France & Le Luc & bords de chaı & $\mathrm{P}$ & Yes \\
\hline France & Beaupuy (près Toulouse), alt. 200 m. & n.a. & $\mathrm{P}$ & No \\
\hline France & Canetto près Bonifacio & Lieux incultes & $P$ & No \\
\hline France & Hyères & n.a. & $P$ & No \\
\hline France & de la Rochelle a la Palisse (Chte Inf.) & n.a. & $\mathrm{P}$ & No \\
\hline France & Magnil près Luçon (Vendée) & n.a. & $P$ & Yes \\
\hline France & Collines de la Gardiole, prèrs Mireval & n.a. & $P$ & No \\
\hline France & Environs de Nice & n.a. & $P$ & Yes \\
\hline France & Béziers: Pradines le Haut & n.a. & $P$ & No \\
\hline France & \multicolumn{2}{|c|}{ Mt Agel, S.E Face above Grand Corniche, n.a. } & $\mathrm{P}$ & No \\
\hline France & Pont de la Fou & n.a. & $P$ & No \\
\hline France & \multicolumn{2}{|c|}{ Mollans-sur-Ouvèze (Drôme): ancienne v Flanc sud du 1} & $\mathrm{P}$ & Yes \\
\hline France & Narbonne & n.a. & $\mathrm{P}$ & No \\
\hline France & Narbonne & n.a. & $\mathrm{P}$ & No \\
\hline France & Fontfroid, Pyrénées Orientales & n.a. & $P$ & No \\
\hline France & Perpignan, Pyrénées Orientales & n.a. & $P$ & No \\
\hline France & Perpignan & n.a. & $P$ & No \\
\hline France & Toulon & n.a. & $\mathrm{P}$ & No \\
\hline
\end{tabular}




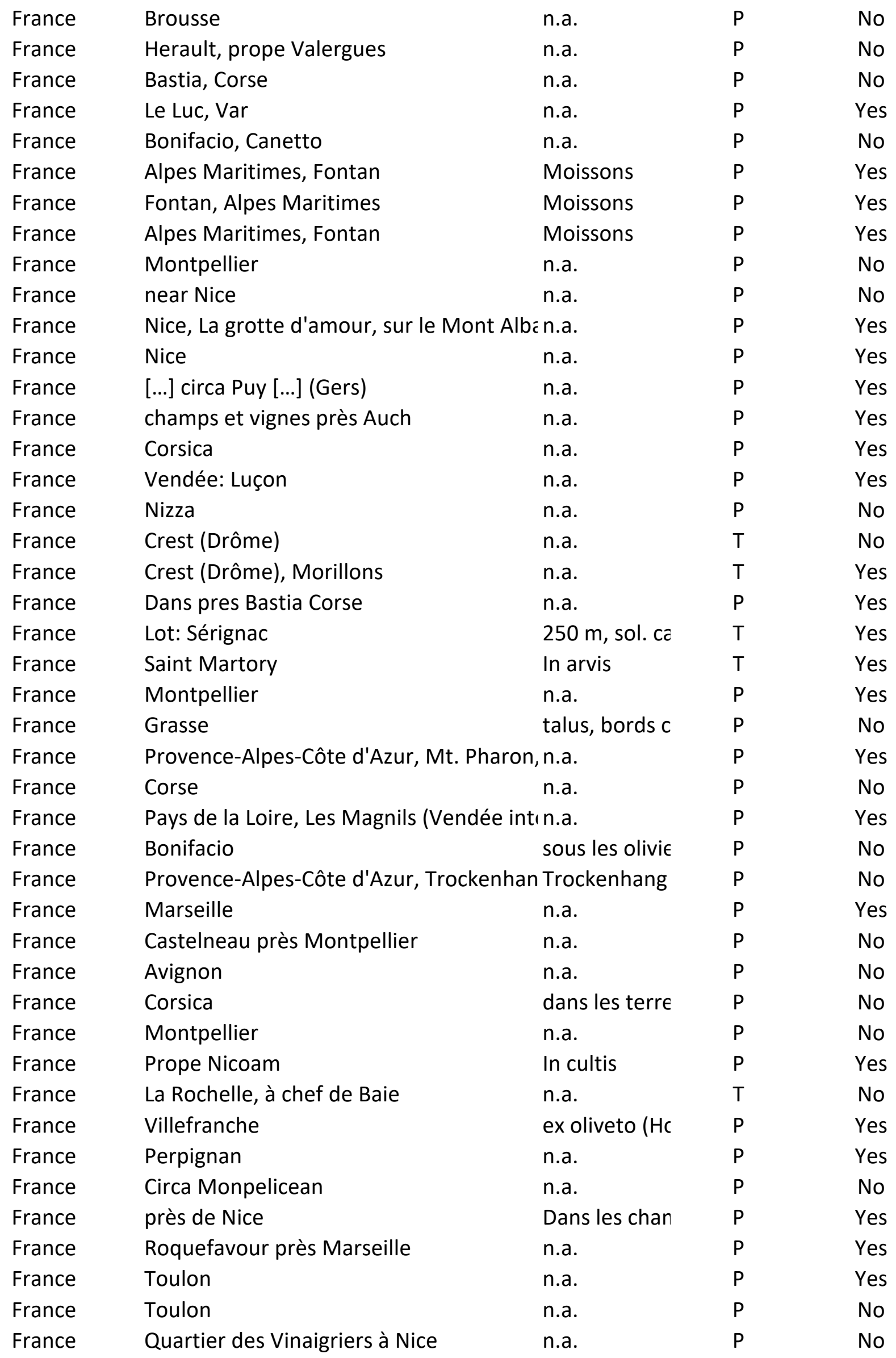




\begin{tabular}{|c|c|c|c|c|}
\hline France & Bords du Lez près Montpellier & n.a. & $\mathrm{P}$ & No \\
\hline France & Mauguio (Hérault), à Mézouls & n.a. & $P$ & Yes \\
\hline France & Menton, dans les environs Cap Martin & n.a. & $P$ & No \\
\hline France & Toulon & n.a. & $\mathrm{P}$ & Yes \\
\hline France & \multicolumn{2}{|c|}{ Lot-et-Garonne, Chabrity, commune de F Champs des c } & $\mathrm{P}$ & No \\
\hline France & \multicolumn{2}{|c|}{ Environs de Marseille (Bouches du Rhôn€ Champs } & $P$ & No \\
\hline France & Près Marseille (Bouches du Rhône) & Champs aux c & $P$ & No \\
\hline France & Cannes & sous les olivi $\epsilon$ & $P$ & Yes \\
\hline France & Var: Draguignan & n.a. & $P$ & Yes \\
\hline France & \multicolumn{2}{|c|}{ Bord moyenne corniche entre Monaco ein.a. } & $P$ & No \\
\hline France & Fontaine de Vaucluse & n.a. & $\mathrm{P}$ & No \\
\hline France & Var: plan d'Aups & n.a. & $P$ & No \\
\hline France & Var: Aups & n.a. & $P$ & No \\
\hline France & Bouches du Rhône, Montmajour & n.a. & $\mathrm{T}$ & No \\
\hline France & Lourmarin & n.a. & $\mathrm{P}$ & No \\
\hline France & Toulon & n.a. & $P$ & No \\
\hline France & Toulon & n.a. & $\mathrm{P}$ & No \\
\hline France & Narbonne & n.a. & $P$ & No \\
\hline France & Montpellier & n.a. & $P$ & No \\
\hline France & Montpellier & n.a. & $\mathrm{T}$ & No \\
\hline France & Département de l'Aude & n.a. & $\mathrm{T}$ & Yes \\
\hline France & Alpes-Maritimes & Terrain vagu€ & $P$ & No \\
\hline France & $6 \mathrm{~km}$. SW of Montpellier to Sete. & Garrigue, terr & $\mathrm{P}$ & No \\
\hline France & Narbonne & n.a. & $\mathrm{P}$ & No \\
\hline France & Corsica & inter segetes & $P$ & No \\
\hline France & Hérault, Montpellier & Champs & $P$ & No \\
\hline France & Environs de Toulon, Fort Rouge & n.a. & $\mathrm{P}$ & Yes \\
\hline France & Menton & Frequent und & $\mathrm{P}$ & Yes \\
\hline France & \multicolumn{2}{|c|}{ Vaucluse, au pied du mt Ventoux, près dı Bords des chá } & $P$ & Yes \\
\hline France & Road near Hôtel d'Italie, Menton & n.a. & $\mathrm{P}$ & Yes \\
\hline France & Mentone. Monte Carlo. & n.a. & $P$ & Yes \\
\hline France & \multicolumn{2}{|c|}{ Route de Ste Anne près Dijon (Côte d'Or) n.a. } & $\mathrm{T}$ & No \\
\hline France & Le Luc (Var) & Bords des chí & $\mathrm{P}$ & Yes \\
\hline France & Beaupuy (près de Toulouse), Hte Garon & Champs et ha & $\mathrm{P}$ & Yes \\
\hline France & Aix-en-Provence & n.a. & $P$ & Yes \\
\hline France & Pyrénées Orientales, Villefranche & $1500 \mathrm{ft}$, grow & $\mathrm{T}$ & No \\
\hline France & Alpes-Maritimes, Cipières & Field edge wi & $\mathrm{P}$ & No \\
\hline France & Avignon & n.a. & $P$ & Yes \\
\hline France & Avignon & n.a. & $P$ & Yes \\
\hline France & Près Uzès, remonte jusqu'à Vigan & Le long des cl & $P$ & Yes \\
\hline France & Lyon. Blés près de St. Alban & n.a. & $\mathrm{T}$ & No \\
\hline France & Bouches-du-Rhône: Aix & bords gazonr & $P$ & Yes \\
\hline France & Béziers & n.a. & $\mathrm{T}$ & Yes \\
\hline France & Les Arisquiers (Hérault) & n.a. & $P$ & No \\
\hline
\end{tabular}




\begin{tabular}{|c|c|c|c|c|}
\hline France & Grasse (AM) & Fossés, lieux & $\mathrm{P}$ & No \\
\hline France & Grasse (AM) & Champs, talu & $\mathrm{P}$ & No \\
\hline France & \multicolumn{2}{|c|}{ Region Provence-Alpes-Cote d'Azur, Der champs incul' } & $P$ & Yes \\
\hline France & Alpes-Maritimes, Villeneuve Loubet & Alt: $39 \mathrm{~m}$. & $\mathrm{T}$ & No \\
\hline France & Alpes-Maritimes, Gourdon & Alt:: $800 \mathrm{~m}$. & $P$ & No \\
\hline France & Alpes Maritimes, Saint Vallier-de-Thiey & Alt: : $800 \mathrm{~m}$. & $P$ & No \\
\hline France & Pova do Conde. Castanheda & n.a. & $P$ & No \\
\hline France & Languedoc-Roussillon, Montpellier. & n.a. & $P$ & Yes \\
\hline France & \multicolumn{2}{|c|}{ Languedoc-Roussillon, Montpellier near In.a. } & $P$ & Yes \\
\hline France & La Rochelle & n.a. & $\mathrm{T}$ & No \\
\hline France & Perpignan, [illegible] & n.a. & $\mathrm{P}$ & No \\
\hline France & Maguelone & n.a. & $P$ & No \\
\hline France & Monte Grosso près de Nice & n.a. & $P$ & No \\
\hline France & Fabrègues & champs & $\mathrm{P}$ & No \\
\hline France & \multicolumn{2}{|c|}{ de la gaillarde à la mosson (? Montpellier bord de chen } & $P$ & No \\
\hline France & Auch (Gers) & n.a. & $\mathrm{T}$ & No \\
\hline France & Toulon, Fort Rouge & n.a. & $P$ & Yes \\
\hline France & Montpellier, Hérault & champs & $\mathrm{P}$ & No \\
\hline France & Montpellier & n.a. & $P$ & No \\
\hline France & Montpellier & champs & $P$ & Yes \\
\hline France & environs d'Angoulême & dans les vign€ & $\mathrm{P}$ & Yes \\
\hline France & Puycasquier (Gers) & n.a. & $P$ & Yes \\
\hline France & près d'Auch & champs et vi६ & $\mathrm{P}$ & Yes \\
\hline France & Richemont (Charente) & échappée de & $\mathrm{T}$ & No \\
\hline France & Point de Chef de Baie (La Rochelle ?) & n.a. & $\mathrm{T}$ & No \\
\hline France & Bourges & décombres aı & $\mathrm{T}$ & No \\
\hline France & Montpellier & n.a. & $\mathrm{P}$ & No \\
\hline France & Montpellier & n.a. & $\mathrm{P}$ & No \\
\hline France & Le Luc (Var) & bords des chí & $P$ & Yes \\
\hline France & Le Luc (Var) & bords de chaı & $P$ & Yes \\
\hline France & coteau du Vinaigrié près Nice & n.a. & $\mathrm{P}$ & Yes \\
\hline France & Pézenas & champs, vignı & $P$ & Yes \\
\hline France & Pézenas & champs, vignı & $\mathrm{T}$ & Yes \\
\hline France & Antibes & n.a. & $\mathrm{P}$ & No \\
\hline France & Auch & n.a. & $P$ & Yes \\
\hline France & Le Luc (Var) & liens incultes & $\mathrm{P}$ & Yes \\
\hline France & Saint Félix de Lodez & n.a. & $\mathrm{P}$ & No \\
\hline France & Lodève au chemin vieux de Lauroux & n.a. & $P$ & No \\
\hline France & Saint Zacharie (Var) & moissons & $P$ & No \\
\hline France & Saint Martin de Londres (Hérault) & bords des che & $P$ & Yes \\
\hline France & Toulon & champs & $\mathrm{P}$ & No \\
\hline France & Montagne de Cette (= Sète), Hérault & n.a. & $\mathrm{P}$ & No \\
\hline France & Montpellier à Montplaisir & haies et char & $P$ & No \\
\hline France & Montpellier & bord des chel & $\mathrm{P}$ & Yes \\
\hline
\end{tabular}




\begin{tabular}{|c|c|c|c|c|}
\hline France & Le Luc (Var) & bords des chí & $\mathrm{P}$ & Yes \\
\hline France & Le Luc (Var) & bords des che & $P$ & Yes \\
\hline France & Cette (Sète) & n.a. & $P$ & No \\
\hline France & \multicolumn{2}{|c|}{ Menton, sur terre italienne bois Grimaldin.a. } & $P$ & No \\
\hline France & Murviel-lez-Montpellier & bords et talus & $\mathrm{P}$ & No \\
\hline France & Montbazin (Hérault) & talus du chen & $\mathrm{P}$ & No \\
\hline France & Mausson & n.a. & $\mathrm{P}$ & Yes \\
\hline France & Béziers, grand fossé du pré-au-nègre & n.a. & $P$ & Yes \\
\hline France & haies à Balaruc & n.a. & $\mathrm{P}$ & No \\
\hline France & bord chemin Montpellier & n.a. & $P$ & No \\
\hline France & La Gaillarde & n.a. & $P$ & No \\
\hline France & Valmaillargues près Montpellier & n.a. & $P$ & No \\
\hline France & Aniane & bords des chí & $\mathrm{P}$ & No \\
\hline France & Grasse & n.a. & $\mathrm{P}$ & No \\
\hline France & Aix (Bouches-du -Rhône) & bords gazonn & $P$ & Yes \\
\hline France & Angoulême & n.a. & $\mathrm{P}$ & Yes \\
\hline France & Cette (Sète) & coteaux mari & $P$ & No \\
\hline France & Saint Guilhem le Désert (Hérault) & n.a. & $\mathrm{P}$ & No \\
\hline France & Seillans (Var) & champs de bl & $P$ & Yes \\
\hline France & Agde (Hérault) & n.a. & $\mathrm{P}$ & No \\
\hline France & \multicolumn{2}{|c|}{ Montpellier, champs de l'école d'agricult n.a. } & $P$ & No \\
\hline France & Nyons (Drôme) & n.a. & $\mathrm{P}$ & No \\
\hline France & Nyons (Drôme) & moissons & $\mathrm{P}$ & No \\
\hline France & La Nouvelle (Aude) & n.a. & $\mathrm{P}$ & No \\
\hline France & La Nouvelle (Aude) & friche & $\mathrm{P}$ & No \\
\hline France & Fronsac (Gironde) & champs de bl & $\mathrm{P}$ & Yes \\
\hline France & La Nouvelle (Aude) & friche & $\mathrm{P}$ & No \\
\hline France & Corse, Bonifacio & sous les olivi $\epsilon$ & $\mathrm{P}$ & No \\
\hline France & La Nouvelle (Aude) & n.a. & $\mathrm{P}$ & No \\
\hline France & environs d'Angoulême & moissons, cot & $P$ & No \\
\hline France & Corse, Cavallo morto près Bonifacio & sous les olivi $\epsilon$ & $\mathrm{P}$ & Yes \\
\hline France & Millau (Aveyron) & ravin (?) & $\mathrm{P}$ & No \\
\hline France & Darnieux & lieux en frichı & $\mathrm{P}$ & No \\
\hline France & Haies à la Coste & n.a. & $\mathrm{P}$ & Yes \\
\hline France & Fréjus & plaine & $\mathrm{P}$ & Yes \\
\hline France & Maraussan \& Ardouane & bord de senti & $P$ & No \\
\hline France & Maraussan \& Ardouane & bord de senti & $\mathrm{T}$ & No \\
\hline France & Béziers, en dessous du canal & pentes herbe & $\mathrm{P}$ & No \\
\hline France & \multicolumn{2}{|c|}{ Savonnières (Indre-et-Loire), route de Vil pieds des mu } & $\mathrm{T}$ & No \\
\hline France & Les Baux du Ventoux & $400 \mathrm{~m}$ & $\mathrm{P}$ & No \\
\hline France & \multicolumn{2}{|c|}{ Montpellier, environs hopital saint-Eloi, ın.a. } & $\mathrm{P}$ & No \\
\hline France & \multicolumn{2}{|c|}{ Montpellier, hauteurs sur la rive droite d n.a. } & $\mathrm{P}$ & Yes \\
\hline France & Grasse (Alpes-Maritimes) & lieux incultes, & $\mathrm{P}$ & No \\
\hline France & Lavalette (près Montpellier) & friches & $\mathrm{P}$ & \\
\hline
\end{tabular}




\begin{tabular}{|c|c|c|c|c|}
\hline France & Murviel-Les-Montpellier (Hérault) & bord de chan & $\mathrm{P}$ & No \\
\hline France & Vaucluse: Les Baux de Vaucluse & n.a. & $\mathrm{P}$ & No \\
\hline France & \multicolumn{2}{|c|}{ Bordeaux; remblais touchant le parc de lin.a. } & $\mathrm{T}$ & No \\
\hline France & Toulon, au bas du Fort rouge & n.a. & $P$ & No \\
\hline France & Toulon, gorges d'Ollioules & n.a. & $\mathrm{P}$ & No \\
\hline France & Nismes & n.a. & $\mathrm{P}$ & No \\
\hline France & Montpellier & n.a. & $\mathrm{P}$ & Yes \\
\hline France & Provence & n.a. & $P$ & No \\
\hline France & \multicolumn{2}{|c|}{ Courbevoie. Environs de Paris. Habitat in n.a. } & $\mathrm{T}$ & No \\
\hline France & Montpellier & n.a. & $P$ & No \\
\hline France & Lourmarin & n.a. & $P$ & No \\
\hline France & Lourmarin & n.a. & $\mathrm{P}$ & No \\
\hline France & Montpellier & n.a. & $\mathrm{P}$ & Yes \\
\hline France & Grasse & n.a. & $P$ & Yes \\
\hline France & Grasse & n.a. & $\mathrm{T}$ & Yes \\
\hline France & Grasse & n.a. & $\mathrm{P}$ & No \\
\hline France & Auch (Gers) & n.a. & $\mathrm{P}$ & No \\
\hline France & Perpignan, à Castel-Roussillon & n.a. & $\mathrm{P}$ & No \\
\hline France & Charente: vignes de Brudy & n.a. & $P$ & No \\
\hline France & Angoulême, Charente & champs cultil & $\mathrm{P}$ & Yes \\
\hline France & \multicolumn{2}{|c|}{ Montpellier, sur le bord du chemin du M n.a. } & $\mathrm{P}$ & No \\
\hline France & Toulon & n.a. & $\mathrm{P}$ & No \\
\hline France & Montpellier & sur le bord dı & $P$ & No \\
\hline France & Montpellier & n.a. & $P$ & No \\
\hline France & Auch & n.a. & $\mathrm{P}$ & Yes \\
\hline France & Bonifacio (Corse) & n.a. & $P$ & No \\
\hline France & Edon (Charente) & observée éga & $\mathrm{P}$ & Yes \\
\hline France & Narbonne & n.a. & $\mathrm{P}$ & Yes \\
\hline France & Nîmes & Bord des char & $\mathrm{T}$ & Yes \\
\hline France & Environs d'Angoulême & dans les chan & $\mathrm{P}$ & No \\
\hline France & St André près Montpellier & n.a. & $\mathrm{P}$ & No \\
\hline France & Montpellier & Bord des chaı & $\mathrm{P}$ & No \\
\hline France & Montpellier, Hérault & Champs & $P$ & Yes \\
\hline France & Montpellier, Hérault & Champs & $P$ & No \\
\hline France & Env. d'Angoulême & n.a. & $\mathrm{P}$ & No \\
\hline France & Montpellier (Hérault) & n.a. & $P$ & No \\
\hline France & Montpellier, champs & n.a. & $P$ & No \\
\hline France & La Rochelle & n.a. & $\mathrm{P}$ & No \\
\hline France & \multicolumn{2}{|c|}{ Dans quelques champs seulement de la c Mr. Letourne } & $\mathrm{P}$ & Yes \\
\hline France & Les Magnils (Vendée) & près Luçon, $d$ & $\mathrm{P}$ & No \\
\hline France & \multicolumn{2}{|c|}{ Lot-et-Garonne. Corne, près de Chabrity, n.a. } & $\mathrm{P}$ & No \\
\hline France & Saintes (Charente maritime) & Spontanée dì & $P$ & No \\
\hline France & Auch & moissons (spr & $\mathrm{T}$ & No \\
\hline France & Auch & n.a. & $\mathrm{T}$ & Yes \\
\hline
\end{tabular}




\begin{tabular}{|c|c|c|c|c|}
\hline France & Environs de Luçon (Vendée) & n.a. & $P$ & No \\
\hline France & Toulon, les Sablettes & n.a. & $\mathrm{P}$ & No \\
\hline France & Villeneuve les maguelonne (Hérault) & n.a. & $P$ & No \\
\hline France & Luc (Var) & Bords des chí & $\mathrm{P}$ & Yes \\
\hline France & Villanière, collines sèches & Plantes du ve & $P$ & No \\
\hline France & Villanière, collines sèches & Plantes du ve & $P$ & No \\
\hline France & Antibes (Alpes-Maritimes) & n.a. & $P$ & No \\
\hline France & Montredon près Marseille & n.a. & $P$ & No \\
\hline France & Montredon: collines & n.a. & $\mathrm{P}$ & No \\
\hline France & St Pons (Bouches du Rhône) & Vallon de St F & $P$ & No \\
\hline France & St Pons (Bouches du Rhône) & Vallon de St F & $\mathrm{T}$ & No \\
\hline France & Toulon & n.a. & $P$ & No \\
\hline France & Nice (Alpes Maritimes) & champs incul 1 & $P$ & No \\
\hline France & Nice & Champs incul & $\mathrm{P}$ & No \\
\hline France & Nice & Champs incul & $P$ & No \\
\hline France & Nice & Champs incul & $P$ & Yes \\
\hline France & Nice & Champs incul & $\mathrm{P}$ & Yes \\
\hline France & Béziers & n.a. & $P$ & No \\
\hline France & Rivesaltes & n.a. & $P$ & No \\
\hline France & \multicolumn{2}{|c|}{ Aveyron: gorges d'Estrabols, $\mathrm{Cm}$ de Salva n.a. } & $\mathrm{T}$ & Yes \\
\hline France & Cavaillon, Vaucluse & n.a. & $\mathrm{P}$ & No \\
\hline France & \multicolumn{2}{|c|}{ Montagne de St Jacques sur Cavaillon (Vin.a. } & $P$ & No \\
\hline France & Grabels & moissons à $\mathrm{G}$ & $P$ & No \\
\hline France & Ile Ste Marguerite & n.a. & $P$ & No \\
\hline France & \multicolumn{2}{|c|}{ Sous les oliviers, près de Menton (Alpes-In.a. } & $\mathrm{P}$ & No \\
\hline France & Tayac, les Eyzies (Dordogne) & n.a. & $P$ & Yes \\
\hline France & \multicolumn{2}{|c|}{ Ile verte près La Ciotat, Bouches du Rhônn.a. } & $P$ & No \\
\hline France & Vaucluse & n.a. & $P$ & No \\
\hline France & près Nice & Iter italicum & $P$ & No \\
\hline France & Corse, Bastia & colline sèche & $\mathrm{P}$ & No \\
\hline France & Nemours & n.a. & $\mathrm{P}$ & No \\
\hline France & Agen & n.a. & $P$ & No \\
\hline France & Crest (Drôme) & moissons & $\mathrm{T}$ & No \\
\hline France & Crest & moissons & $\mathrm{T}$ & No \\
\hline France & Orange (Vaucluse) & Mourrarouge & $P$ & No \\
\hline France & Les Magnils (Vendée) & près Luçon, d & $P$ & No \\
\hline France & Cheverny (Loir et Cher) & Herbier de pli & $P$ & No \\
\hline France & Vaucluse & bord de la roı & $\mathrm{P}$ & No \\
\hline France & Jourdan & sans doute éc & $\mathrm{T}$ & No \\
\hline France & Toulon (Var) & lieux incultes & $\mathrm{P}$ & No \\
\hline France & Gorniès (Hérault) & moissons, prè & $P$ & No \\
\hline France & Montpellier & n.a. & $P$ & Yes \\
\hline France & Les Magnisles, près Luçon & n.a. & $\mathrm{P}$ & Yes \\
\hline France & Le Luc (Var), haies et lieux incultes & n.a. & $\mathrm{P}$ & Yes \\
\hline
\end{tabular}




\begin{tabular}{|c|c|c|c|c|}
\hline France & Le Luc (Var), haies et lieux incultes & n.a. & $\mathrm{T}$ & Yes \\
\hline France & Clermont & subspontanéı & $\mathrm{T}$ & No \\
\hline France & Flassan & moissons & $P$ & No \\
\hline France & Beaupuy (près de Toulouse) & n.a. & $P$ & No \\
\hline France & Montagne de Celle (Hérault) & n.a. & $\mathrm{P}$ & No \\
\hline France & Beaupuy (Haute Garonne) & Champs et ha & $\mathrm{P}$ & No \\
\hline France & Beaupuy (Haute Garonne) & Champs et ha & $P$ & No \\
\hline France & Beaupuy (Haute Garonne) & Champs et ha & $\mathrm{P}$ & No \\
\hline France & Millau & n.a. & $P$ & No \\
\hline France & Millau & n.a. & $\mathrm{T}$ & No \\
\hline France & Monsempron Libos (Lot-et-Garonne) & n.a. & $\mathrm{T}$ & Yes \\
\hline France & Drap (06) & région littora & $P$ & No \\
\hline France & Sérignan (Vaucluse) & Bord des chaı & $\mathrm{P}$ & No \\
\hline France & Fontan & Alpes-Maritin & $\mathrm{P}$ & No \\
\hline France & Fontan & moissons & $\mathrm{P}$ & Yes \\
\hline France & Belle lle en Mer (Morbihan) & provenant de & $\mathrm{T}$ & Yes \\
\hline France & \multicolumn{2}{|c|}{ Carcassone (Aude), moissons vers St Jear n.a. } & $P$ & No \\
\hline France & Annecy & carrières & $\mathrm{T}$ & Yes \\
\hline France & Pointe du Chef de Baie & n.a. & $\mathrm{P}$ & No \\
\hline France & Montboron (Alpes Maritimes) & région littora & $P$ & No \\
\hline France & Fontaine de Vaucluse & n.a. & $\mathrm{P}$ & No \\
\hline France & Nice (Alpes Maritimes) & région littora & $\mathrm{P}$ & No \\
\hline France & Antibes & ça et là dans | & $\mathrm{P}$ & No \\
\hline France & Mayenne & sur le chemin & $\mathrm{T}$ & No \\
\hline France & Salavre (Ain) & terre sablonn & $P$ & No \\
\hline France & Corse: Aleria, domaine de Casabianda & n.a. & $\mathrm{P}$ & No \\
\hline France & Fronsac (Gironde) & les champs d & $\mathrm{P}$ & No \\
\hline France & La nouvelle (Aude) & var. minor Bo & $\mathrm{P}$ & No \\
\hline France & Arsyron: Peyre, route de Comprégnac & Var. minor $\mathrm{BC}$ & $\mathrm{T}$ & No \\
\hline France & Chartres (Eure et Loir) & chemin, long، & $\mathrm{T}$ & No \\
\hline France & Millau (Aveyron) & côte de St Est & $P$ & No \\
\hline France & Millau (Aveyron) & côte de St Est & $\mathrm{T}$ & No \\
\hline France & La Nouvelle (Aude) & var. minor Bo & $P$ & No \\
\hline France & Angoulême & moissons calc & $\mathrm{P}$ & No \\
\hline France & Dord. Champs de Granbges & n.a. & $P$ & No \\
\hline France & Aude: La Nouvelle & n.a. & $\mathrm{P}$ & No \\
\hline France & Grasse (Alpes Maritimes) & de Mouans Si & $\mathrm{P}$ & No \\
\hline France & St Germain (Seine et Oise) & n.a. & $\mathrm{T}$ & No \\
\hline France & St Germain (Seine et Oise) & n.a. & $\mathrm{T}$ & No \\
\hline France & \multicolumn{2}{|c|}{ Le Val d'CEuf, Cne de Mougins (Alpes Maın.a. } & $\mathrm{P}$ & No \\
\hline France & Cette & n.a. & $P$ & No \\
\hline France & Cette & n.a. & $\mathrm{T}$ & No \\
\hline France & Environs de Marseille & (Sté bat. de F & $P$ & Yes \\
\hline France & Tarbes (Hautes Pyrénées) & route de Pau & $\mathrm{T}$ & No \\
\hline
\end{tabular}




\begin{tabular}{|c|c|c|c|c|}
\hline France & Sète (Hérault) & Montagne de & $\mathrm{T}$ & No \\
\hline France & Sannois (S. et O.) & n.a. & $\mathrm{T}$ & No \\
\hline France & Montpellier (Hérault) & haies & $P$ & No \\
\hline France & Saint-Tropez, Sainte-Anne & n.a. & $P$ & No \\
\hline France & Duran près Auch (Gers) & n.a. & $\mathrm{P}$ & No \\
\hline France & Entre Béziers et Agde, garrigues de Preig $\mid r$ & n.a. & $\mathrm{P}$ & No \\
\hline France & Saint-Joseph, Saint-Tropez. Var $\quad r$ & n.a. & $\mathrm{P}$ & No \\
\hline France & Saint-Gilles / Gard & n.a. & $\mathrm{T}$ & No \\
\hline France & Ezanville & champ en bol & $\mathrm{T}$ & No \\
\hline France & Béziers (Hérault) & tertres herbe & $P$ & No \\
\hline France & Béziers (Hérault) & tertres herbe & $P$ & No \\
\hline France & près de Chaix, Vendée & sur la route $d$ & $\mathrm{~T}$ & Yes \\
\hline France & La Clarté, près Perros Guirrec & bord des chel & $\mathrm{T}$ & No \\
\hline France & Brignoles (Var) & coteaux vers & $\mathrm{P}$ & No \\
\hline France & Hyères & n.a. & $P$ & Yes \\
\hline France & Villeneuve les Avignon & n.a. & $\mathrm{T}$ & No \\
\hline France & Nice (Alpes Maritimes) & champs sous & $\mathrm{P}$ & No \\
\hline France & Gordes & n.a. & $P$ & No \\
\hline France & Plazac (Dordogne) & route de Bar & $\mathrm{T}$ & No \\
\hline France & Gordes & n.a. & $\mathrm{P}$ & No \\
\hline France & La Valentine (Bouches du Rhône) & friche à Mont & $\mathrm{P}$ & No \\
\hline France & La Valentine (Bouches du Rhône) & friche à Mont & $\mathrm{P}$ & No \\
\hline France & Bandol (Var) & Champs \& mc & $\mathrm{T}$ & No \\
\hline France & Laghet (Alpes maritimes) & route de la $\mathrm{Tr}$ & $\mathrm{P}$ & Yes \\
\hline France & Toulon & n.a. & $\mathrm{P}$ & No \\
\hline France & Sainte Baume (Provence) & n.a. & $\mathrm{P}$ & No \\
\hline France & Montpellier & n.a. & $\mathrm{P}$ & No \\
\hline France & Cap d'Ail (Alpes maritimes) & garrigue le lo| & $\mathrm{P}$ & No \\
\hline France & Saintes (Charente maritime) & n.a. & $\mathrm{T}$ & No \\
\hline France & Montpellier (Hérault) & champs & $\mathrm{P}$ & No \\
\hline France & Montpellier (Hérault) & Plan des 4 sei & $P$ & No \\
\hline France & près d'Angoulême (Charente) & Champs & $\mathrm{P}$ & No \\
\hline France & Le Val (Var) & n.a. & $P$ & No \\
\hline France & Saint Maximin (Var) & entre la ville & $\mathrm{P}$ & No \\
\hline France & Sucy-en-Brie (Seine-et-Marne) & Moissons & $\mathrm{T}$ & No \\
\hline France & Seine-Port & Echappé jardi & $\mathrm{T}$ & No \\
\hline France & Aurons (Bouches du Rhône) & garrigues et c & $\mathrm{P}$ & No \\
\hline France & Vauvenargues & près du Chât€ & $\mathrm{P}$ & No \\
\hline France & Charente maritime, Lhoumeau & moissons & $\mathrm{P}$ & No \\
\hline France & Laleu (Charente Maritime) & dans un char & $\mathrm{P}$ & No \\
\hline France & St Clément (Hérault) & n.a. & $\mathrm{P}$ & No \\
\hline France & \multicolumn{2}{|l|}{ Vaucluse: Bédoin, chemin vers la ferme [ n.a. } & $\mathrm{P}$ & Yes \\
\hline France & Puymoyen (Charente) & champ pierre & $\mathrm{P}$ & No \\
\hline France & Athis-Mons (Essone), terrain encore vagı $\iota$ & un seul indivi & $\mathrm{T}$ & No \\
\hline
\end{tabular}




\begin{tabular}{|c|c|c|c|c|}
\hline France & \multicolumn{2}{|c|}{ Montelimar (Ardèche-Drôme). La Garde ، n.a. } & $P$ & No \\
\hline France & \multicolumn{2}{|c|}{ Montelimar (Ardèche-Drôme). La Garde ، n.a. } & $P$ & No \\
\hline France & Notre Dame de Pau (py or) & n.a. & $\mathrm{P}$ & Yes \\
\hline France & Aveyron: Canton de Villeneuve, environ & n.a. & $\mathrm{T}$ & Yes \\
\hline France & Corse. Bonifacio a Canetto & n.a. & $\mathrm{P}$ & Yes \\
\hline France & Gallica. Luçon & n.a. & $\mathrm{P}$ & Yes \\
\hline France & \multicolumn{2}{|c|}{ Provence-Alpes-Cote d'Azur-Menton. Roı n.a. } & $\mathrm{T}$ & No \\
\hline France & \multicolumn{2}{|c|}{ Coutronne (massif de la S: tb=Baume) B. n.a. } & $\mathrm{P}$ & No \\
\hline France & Menton. Alp. Mar & n.a. & $\mathrm{P}$ & Yes \\
\hline France & Bretagne, Frankrike, Benodet & n.a. & $\mathrm{T}$ & No \\
\hline France & Bretagne, Frankrike, Benodet & n.a. & $\mathrm{T}$ & No \\
\hline France & Nice, sur le Mont Alban & n.a. & $\mathrm{P}$ & No \\
\hline France & champs, Marseille, bouches du rhône & n.a. & $\mathrm{P}$ & No \\
\hline France & \multicolumn{2}{|c|}{ Corse: sous les oliviers de Cavallomorto kn.a. } & $\mathrm{P}$ & Yes \\
\hline France & \multicolumn{2}{|c|}{ Provence-Alpes-Côte d'Azur: Bouches-du n.a. } & $\mathrm{P}$ & No \\
\hline France & \multicolumn{2}{|c|}{ Languedoc-Roussillon: Hérault, Montpell n.a. } & $\mathrm{P}$ & No \\
\hline France & \multicolumn{2}{|c|}{ Provence-Alpes-Côte d'Azur: Var, champ: Lieux pierreu: } & $P$ & \\
\hline France & \multicolumn{2}{|c|}{ Provence-Alpes-Côte d'Azur: Bouches-du Lieux incultes } & $\mathrm{P}$ & \\
\hline France & \multicolumn{2}{|c|}{ Provence-Alpes-Côte d'Azur: Bouches-du Lieux incultes } & $\mathrm{T}$ & \\
\hline France & \multicolumn{2}{|c|}{ Pays de la Loire: Vendée, Les Magnils [Le Inter segetes. } & $\mathrm{P}$ & \\
\hline France & \multicolumn{2}{|c|}{ Provence-Alpes-Côte d'Azur: Alpes-Marit n.a. } & $\mathrm{P}$ & \\
\hline France & \multicolumn{2}{|c|}{ Provence-Alpes-Côte d'Azur: Alpes-Marit Moissons } & $\mathrm{P}$ & \\
\hline France & \multicolumn{2}{|c|}{ Provence-Alpes-Côte d'Azur: Alpes-Marit n.a. } & $\mathrm{P}$ & \\
\hline France & \multicolumn{2}{|c|}{ Champs à Gleizé, près Villefranche-s Saor n.a. } & $\mathrm{T}$ & \\
\hline France & Le Luc (Var) & haies, lieux in & $\mathrm{P}$ & \\
\hline France & Bouches du Rhone: Luynes & champs et lie & $\mathrm{P}$ & \\
\hline France & Perpignan, campus Univ & n.a. & $\mathrm{P}$ & \\
\hline
\end{tabular}




\section{GPS}

43.646111, 0.586667

43.710287, 7.305119

43.610556, 3.876667

48.573405, 7.752111

44.732496, -0.462423

43.021111, 3.043889

41.387174, 9.159269

47.384882, 0.554293

43.296389, 5.483333

43.660153, 6.926492

45.764043, 4.835659

45.744161, -0.631667

43.603911, 1.448975

42.974942, 9.429053

43.154306, 5.945556

43.515225, 5.306681

43.621975, 5.299489

43.621975, 5.299489

45.926661, -1.008539

42.692003, 9.450392

42.692003, 9.450392

42.692003, 9.450392

43.100242, 6.133344

43.891711, 3.632081

43.393722, 6.316657

43.650664, 1.55445

41.422981, 9.220775

43.125556, 6.13

46.162808, -1.205111

46.572727, -0.964886

43.499933, 3.756367

43.710173, 7.261953

43.380042, 3.211053

43.766328, 7.432408

42.800797, 2.498919

44.239147, 5.126847

43.184277, 3.003078

43.184277, 3.003078

43.127395, 2.898443

42.688659, 2.894833

42.688659, 2.894833

43.124228, 5.928 
$43.718565,2.072531$

43.667285, 4.061846

$42.692003,9.450392$

43.393722, 6.316657

41.422981, 9.220775

$44.002722,7.554131$

$44.002722,7.554131$

$44.002722,7.554131$

43.610556, 3.876667

43.710173, 7.261953

43.706718, 7.298076

$43.710173,7.261953$

44.658341, 0.060664

43.646111, 0.586667

42.039604, 9.012893

46.454772, -1.167062

$43.710173,7.261953$

$44.728275,5.024072$

44.728275, 5.024072

42.692003, 9.450392

43.920553, 1.022943

43.143227, 0.930699

43.610556, 3.876667

43.660153, 6.926492

43.154306, 5.945556

42.039604, 9.012893

46.572727, -0.964886

41.387174, 9.159269

43.710173, 7.261953

43.3, 5.383333333

43.638755, 3.913851

43.949317, 4.805528

42.039604, 9.012893

43.610556, 3.876667

$43.710173,7.261953$

46.160329, -1.151139

42.529047, 2.248169

42.688659, 2.894833

43.610556, 3.876667

43.710173, 7.261953

43.545232, 5.286626

43.124228, 5.928

43.124228, 5.928

43.710287, 7.305119 
43.604431, 3.736497

43.618286, 3.967411

43.774444, 7.4975

43.124228, 5.928

$44.250278,0.631942$

$43.3,5.383333333$

43.3, 5.383333333

43.553876, 6.996755

43.535682, 6.449755

43.721303, 7.401067

43.923056, 5.127778

43.618166, 6.206349

43.618166, 6.206349

43.702457, 4.666115

43.763477, 5.362582

43.124228, 5.928

43.124228, 5.928

43.184277, 3.003078

43.610556, 3.876667

43.610556, 3.876667

43.123319, 2.433942

43.907481, 7.155104

43.515294, 3.805496

43.184277, 3.003078

42.039604, 9.012893

43.610556, 3.876667

43.142984, 5.916093

43.774444, 7.4975

44.124667, 5.214217

43.774444, 7.4975

43.774444, 7.4975

47.322047, 5.04148

43.393722, 6.316657

43.650664, 1.55445

$43.529722,5.447222$

42.529047, 2.248169

43.783361, 6.955305

43.949317, 4.805528

43.949317, 4.805528

44.01211, 4.419946

$45.732448,4.88758$

$43.529722,5.447222$

43.344167, 3.215556

$43.468969,3.811328$ 
43.660153, 6.926492

43.660153, 6.926492

43.660153, 6.926492

43.657892, 7.122337

43.719923, 6.979338

43.698521, 6.847956

43.454229, -0.994301

43.616667, 3.8833333

43.627139, 3.814167

46.160329, -1.151139

42.688659, 2.894833

43.511944, 3.883889

42.501667, 8.922778

43.549722, 3.778889

43.625833, 3.8225

43.646111, 0.586667

43.142984, 5.916093

43.610556, 3.876667

43.610556, 3.876667

43.610556, 3.876667

45.648377, 0.156237

$43.745833,0.7475$

43.646111, 0.586667

45.721944, -0.365

46.160329, -1.151139

47.080833, 2.398611

43.610556, 3.876667

43.610556, 3.876667

43.393722, 6.316657

$43.393722,6.316657$

43.710287, 7.305119

43.461389, 3.423056

$43.461389,3.423056$

$43.580278,7.125$

$43.646111,0.586667$

43.393722, 6.316657

43.662778, 3.460556

43.733611, 3.313889

43.384228, 5.710687

43.791551, 3.732024

43.124228, 5.928

43.407778, 3.700556

43.610556, 3.876667

43.610556, 3.876667 
43.393722, 6.316657

43.393722, 6.316657

43.407778, 3.700556

43.774444, 7.4975

43.604431, 3.736497

43.516111, 3.694167

48.449572, -1.061482

43.344167, 3.215556

43.46, 3.685278

43.610556, 3.876667

43.625833, 3.8225

43.659167, 3.816389

43.684167, 3.586667

$43.660153,6.926492$

43.529722, 5.447222

$45.648377,0.156237$

$43.407778,3.700556$

43.734167, 3.548889

$43.636111,6.643333$

43.308611, 3.476667

43.610556, 3.876667

44.361667, 5.14

44.361667, 5.14

43.021111, 3.043889

43.021111, 3.043889

44.924167, -0.271111

$43.021111,3.043889$

41.387174, 9.159269

43.021111, 3.043889

45.648377, 0.156237

41.386944, 9.159167

44.100556, 3.077778

43.803006, 3.755055

43.791975, 3.739444

$43.433056,6.736944$

43.368611, 3.157222

43.368611, 3.157222

43.344167, 3.215556

$47.3475,0.548333$

44.124667, 5.214217

43.629206, 3.865351

43.632037, 3.891778

43.660153, 6.926492

$43.645052,3.876418$ 
43.604431, 3.736497

44.124667, 5.214217

44.819444, -0.550556

43.142984, 5.916093

43.139901, 5.847652

43.836699, 4.360054

43.610556, 3.876667

44.014494, 6.211644

48.900552, 2.25929

43.610556, 3.876667

43.763477, 5.362582

43.763477, 5.362582

43.610556, 3.876667

43.660153, 6.926492

43.660153, 6.926492

$43.660153,6.926492$

43.646111, 0.586667

42.712812, 2.950954

45.751996, 0.153476

45.648377, 0.156237

43.610556, 3.876667

43.124228, 5.928

43.610556, 3.876667

43.610556, 3.876667

43.646111, 0.586667

41.387174, 9.159269

45.487706, 0.355149

43.184277, 3.003078

$43.836699,4.360054$

45.648377, 0.156237

43.652984, 3.508645

43.610556, 3.876667

43.610556, 3.876667

43.610556, 3.876667

45.648377, 0.156237

43.610556, 3.876667

43.610556, 3.876667

46.160329, -1.151139

46.572727, -0.964886

46.572727, -0.964886

$44.255647,0.629892$

45.744161, -0.631667

$43.646111,0.586667$

$43.646111,0.586667$ 
46.454772, -1.167062

43.124228, 5.928

43.53324, 3.861616

43.393722, 6.316657

43.341893, 2.361444

43.341893, 2.361444

43.580278, 7.125

43.234312, 5.361918

43.234312, 5.361918

44.392471, 6.628415

44.392471, 6.628415

43.124228, 5.928

43.710173, 7.261953

43.710173, 7.261953

43.710173, 7.261953

43.710173, 7.261953

43.710173, 7.261953

43.344167, 3.215556

42.768583, 2.871763

44.461111, 1.902778

43.836605, 5.040781

43.834552, 5.034722

43.647718, 3.797514

43.519925, 7.0485

43.774444, 7.4975

44.943275, 1.006074

43.159943, 5.617941

44.056505, 5.143207

43.710173, 7.261953

42.692003, 9.450392

48.267043, 2.692611

44.203142, 0.616363

$44.728275,5.024072$

44.728275, 5.024072

44.138099, 4.807511

46.572727, -0.964886

47.49973, 1.46013

44.056505, 5.143207

44.900794, 4.802146

43.124228, 5.928

43.891711, 3.632081

43.610556, 3.876667

46.454772, - 1.167062

$43.393722,6.316657$ 
43.393722, 6.316657

45.777222, 3.087025

44.098889, 5.242222

43.650664, 1.55445

43.661506, 3.341121

43.650664, 1.55445

43.650664, 1.55445

43.650664, 1.55445

44.100556, 3.077778

44.100556, 3.077778

44.504031, 0.929523

43.754365, 7.320831

43.282801, 3.279651

$44.002722,7.554131$

$44.002722,7.554131$

47.336421, -3.181043

43.212161, 2.353663

45.899247, 6.129384

46.147551, -1.210261

43.689829, 7.301271

43.923056, 5.127778

$43.710173,7.261953$

43.580278, 7.125

48.306124, -0.620935

46.364005, 5.346603

$42.104248,9.512429$

44.924167, -0.271111

43.021111, 3.043889

44.09216, 2.999246

48.443854, 1.489012

44.100556, 3.077778

44.100556, 3.077778

43.021111, 3.043889

45.648377, 0.156237

45.146949, 0.757221

43.021111, 3.043889

43.660153, 6.926492

48.565556, 2.06

48.565556, 2.06

43.602332, 7.006491

43.407778, 3.700556

43.407778, 3.700556

43.296482, 5.36978

43.232951, 0.078082 
43.407778, 3.700556

48.970782, 2.256869

43.610556, 3.876667

43.267681, 6.640711

43.646111, 0.586667

$43.326263,3.377885$

43.267681, 6.640711

$43.677079,4.433703$

49.038069, 2.360491

43.344167, 3.215556

43.344167, 3.215556

$46.433063,-0.853614$

$48.81833,-3.473795$

$43.40655,6.061187$

43.125556, 6.13

43.96341, 4.796959

$43.710173,7.261953$

43.911315, 5.200176

45.036291, 1.037587

43.911315, 5.200176

$43.296389,5.483333$

$43.296389,5.483333$

43.136418, 5.754186

43.75299, 7.381835

43.124228, 5.928

43.334817, 5.756087

43.610556, 3.876667

43.72208, 7.405839

45.744161, -0.631667

43.610556, 3.876667

43.610556, 3.876667

45.648377, 0.156237

43.438959, 6.071791

43.559215, 5.393301

$48.770878,2.522163$

48.556855, 2.550114

43.664817, 5.155542

43.555284, 5.602142

45.655485, 0.161095

45.864332, -0.506285

43.683448, 3.840085

44.124667, 5.214217

$45.61349,0.18178$

48.709298, 2.38479 
44.393179, 4.753137

44.393179, 4.753137

43.2951, -0.370797

44.461111, 1.902778

41.422981, 9.220775

46.454772, -1.167062

43.774444, 7.4975

43.598064, 5.445938

43.774444, 7.4975

47.875568, -4.106373

47.875568, -4.106373

43.700818, 7.300177

43.296482, 5.369780

41.406445, 9.169541

43.296482, 5.36978

43.610556, 3.876667

43.124228, 5.928

43.404811, 5.053728

43.404811, 5.053728

46.454772, - 1.167062

43.774444, 7.4975

$44.002722,7.554131$

43.774444, 7.4975

45.988917, 4.696849

43.393722, 6.316657

$47.384882,0.554293$

42.682090, 2.899657 